\title{
Bayesian VAR Forecasts, Survey Information and Structural Change in the Euro Area
}

\section{Gergely Ganics ${ }^{1}$ and Florens Odendahl ${ }^{2}$,}

\author{
October 2019, WP \#733
}

\begin{abstract}
We incorporate external information extracted from the European Central Bank's Survey of Professional Forecasters into the predictions of a Bayesian VAR, using entropic tilting and soft conditioning. The resulting conditional forecasts significantly improve the plain BVAR point and density forecasts. Importantly, we do not restrict the forecasts at a specific quarterly horizon, but their possible paths over several horizons jointly, as the survey information comes in the form of one- and two-year-ahead expectations. Besides improving the accuracy of the variable that we target, the spillover effects to "other-than-targeted" variables are relevant in size and statistically significant. We document that the baseline BVAR exhibits an upward bias for GDP growth after the financial crisis and our results provide evidence that survey forecasts can help mitigate the effects of structural breaks on the forecasting performance of a popular macroeconometric model.
\end{abstract}

Keywords: Survey of Professional Forecasters, Density forecasts, Entropic tilting, Soft conditioning. JEL classification: C53; C32; E37

\footnotetext{
${ }^{1}$ Banco de España, gergely.ganics@bde.es

${ }^{2}$ Banque de France, Monetary policy and financial research dept., florens.odendahl@,banque-france.fr;
}

We thank Anton Grui, Michele Lenza, Giulia Sestieri, and participants at the IXth Zaragoza Workshop in Time Series Econometrics, the BigNOMICS Workshop on Big Data and Economic Forecasting 2019 (Ispra), the 39th International Symposium on Forecasting (Thessaloniki), the IAAE (Cyprus), the CEF (Ottawa), the Banco de Espana and the Banque de France for useful comments. The views expressed herein are those of the authors and should not be attributed to the Banco de España, the Banque de France, or the Eurosystem.

Working Papers reflect the opinions of the authors and do not necessarily express the views of the Banque de France. This document is available on publications.banque-france.fr/en 


\section{NON-TECHNICAL SUMMARY}

Central banks conduct surveys in which they ask forecasters of the private sector for their predictions. It is known that, when averaging the predictions of these survey panelists, i.e. the "consensus forecast", these predictions also provide precise forecasts of key macroeconomic variables. Unfortunately, due to the limited scope of the surveys, to which participation is voluntary, these survey predictions are usually not available for all forecast horizons or variables of interest. On the other hand, it is straightforward to generate predictions from statistical models for any variable at any forecast horizon. The recent forecasting literature, therefore, tries to bridge this gap by incorporating external information into statistical models, to benefit from the quality of survey forecasts beyond the scope of the surveys itself.

In this paper, we use two existing methods, called "entropic tilting", and "soft conditioning", to incorporate external information, coming from survey forecasts, into the forecasts of a Vector Autoregressive (VAR) model. A VAR model is a statistical model that allows to model a set of variables in a dynamic form. As it allows modelling the dynamics of more than one variable it is a multi-variate model, i.e. we can model the dynamics of real GDP growth, HICP inflation and the unemployment rate jointly in a system of equations. The model is called dynamic because it allows for a relationship between past, future and present values of the variables.

The variables we use in the baseline VAR are real GDP growth, HICP inflation, the unemployment rate and the Euribor (a benchmark for the interbank interest rate). The VAR provides us with forecasts of the mean, i.e. a "point forecast", and also provides us with a forecast of the uncertainty surrounding the mean, which we subsequently call a "density forecast".

We find that incorporating one- and two-year-ahead survey expectations on GDP growth, HICP inflation and the unemployment rate considerably improves predictive accuracy. Improvements are statistically and economically significant and the results hold for both point and density forecasts. In addition to improvements in the forecast accuracy of the variable for which external information is used, spillover effects to the other variables are positive in terms of predictive accuracy, often statistically significant and qualitatively similar across the two methods. We provide evidence that around the two recent euro area recessions, as well as the slow recovery thereafter, SPF panelists predicted GDP growth more accurately than a VAR model, hence integrating this information into the econometric model's forecasts leads to considerable gains. Importantly, our results do not advocate abandoning VARs for forecasting, as the "tilting procedure" relies directly on the dynamics of the VAR. Instead, we consider the incorporation of additional information as a simple way to correct possible misspecifications in an otherwise useful model.

We take the external forecasts from the Survey of Professional Forecasters (SPF), which is conducted quarterly by the ECB. Our sample of SPF forecasts ranges from 1999:Q1 to 2017:Q3. More information about the survey can be found on the website of the ECB.

The first method, entropic tilting, is based on a paper by Robertson et al. (2005). In simple terms, the method allows to impose restrictions on (i) the year-on-year point forecast, i.e. the expected growth over the next year, and also (ii) the uncertainty surrounding this forecast. Because in a dynamic model the values of the variables depend on each other across time, by restricting the year-on-year point forecast and uncertainty, we indirectly impose restrictions on other forecast horizons as well.

The second method, soft conditioning, is based on Waggoner (1999). Originally, the authors aimed at producing conditional forecasts in multivariate models. A conditional forecasts means, for instance, to extract GDP and inflation forecasts, given a specific future path of 
the policy interest rate. In a similar spirit, the method can be used to impose a future path, of say GDP growth, where the path is based on external information.

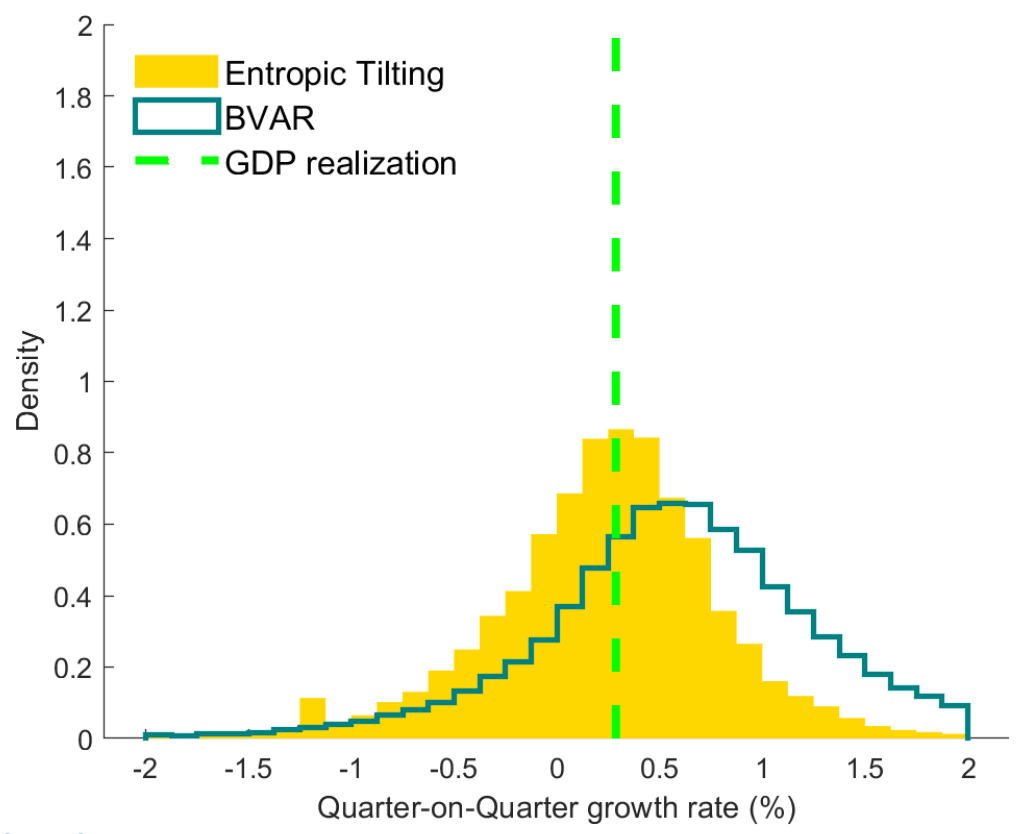

Figure 1: The effect of entropic tilting on the two-quarter-ahead, 2013:Q4 over 2013:Q3, GDP growth forecast.

\title{
Prévisions basées sur des modèles VAR bayésien, informations d'enquêtes et changements structurels dans la zone euro
}

\begin{abstract}
RÉSUMÉ
En utilisant des méthodes dites de "entropic tilting " et de "soft conditioning, nous incorporons dans un modèle VAR bayésien des informations externes provenant des enquêtes menées par la Banque Centrale Européenne auprès des prévisionnistes professionnels. Les prévisions conditionnelles résultantes améliorent considérablement les points et les densités de prévision. Il est important de souligner que nous n'imposons pas de contraintes de prévision sur un horizon trimestriel particulier, mais plutôt sur un horizon annuel, comme les informations fournies par les enquêtes sont disponibles sous la forme d'anticipations à des horizons temporelles d'un et deux ans. Au-delà de fournir une plus grande précision dans la prévision d'une variable cible, il existe des " effets de ricochet " sur les variables autres que celle ciblée. Nous montrons que notre modèle VAR de référence introduit un biais à la hausse sur le taux de croissance du PIB après la crise financière. Nos résultats démontrent que les prévisions recueillies au moyen d'enquêtes peuvent atténuer les effets de ruptures structurelles sur la performance d'un modèle macroeconométrique classique.
\end{abstract}

Mots-clés : Enquêtes des prévisionnistes professionnels, Evaluation des densités de prévisions, Entropic tilting, Soft conditioning.

Les Documents de travail reflètent les idées personnelles de leurs auteurs et n'expriment pas nécessairement la position de la Banque de France. Ils sont disponibles sur publications.banque-france.fr 


\section{Introduction}

Producing reliable forecasts is a central effort of many academics and central banks and it is known that pooling the predictions of survey panelists provides precise forecasts of key macroeconomic variables (see e.g. Ang et al. (2007) and Stark (2010)). However, survey expectations are usually not available for all horizons or variables of interest, and are often limited to point predictions. On the other hand, it is straightforward to generate both point and density forecasts from time series models for any variable at any horizon. The recent literature, therefore, tries to bridge this gap by incorporating external information into econometric models, to benefit from survey forecasts beyond the scope of the surveys itself.

We contribute to this literature by using two existing methods, entropic tilting, and soft conditioning, to incorporate external information into the forecasts of a Bayesian Vector Autoregressive (BVAR) model estimated using real-time euro area data. We find that incorporating one- and two-year-ahead survey expectations on GDP growth, harmonised index of consumper prices (HICP) inflation and the unemployment rate considerably improves predictive accuracy. Improvements are statistically and economically significant and the results hold for both point and density forecasts. In addition to improvements in the forecast accuracy of the variable for which external information is used, spillover effects to the other variables are positive in terms of predictive accuracy, often statistically significant and qualitatively similar across the two methods. We provide evidence that around the two recent euro area recessions, as well as the slow recovery thereafter, SPF panelists predicted GDP growth more accurately than a BVAR model, hence integrating this information into the econometric model's forecasts leads to considerable gains. We consider the incorporation of additional information as a way to correct possible misspecifications in an otherwise useful model and our results do not advocate to abandon using BVARs for forecasting. In contrast, the positive spillover effects imply that not only the conditioning information itself is valuable but also that the BVAR translates the conditioning information on one variable into useful information on other variables.

The underlying econometric model is a four-variable Bayesian VAR with stochastic volatility (Clark and Ravazzolo, 2015), including real GDP growth, HICP inflation, the unemployment rate, and the 3-month Euribor rate. To mimic the actual forecasting environment that forecasters faced at a given point in time, we use real-time data vintages (building on the real-time database by Giannone et al., 2012) for the former three series (the interest rate variable is not revised). Due to a "ragged edge" problem, introduced by the mismatch between the timing of the survey and the data releases, we additionally apply the jumping-off procedure of Faust and Wright (2009) in combination with ECB/Eurosystem staff projections. ${ }^{1}$

To rule out alternative explanations for the improvements over the baseline BVAR, we conduct several robustness checks. First, to account for time-variation in parameters, we produce forecasts using a time-varying parameter BVAR. Second, to account for omitted variables, we produce forecasts using a large (14-variable) BVAR. Third, we use a "dummy-initial-observation" prior to rule out that results are driven by an overfitting of the deterministic component. The model is implemented using the hierarchical BVAR of Giannone et al. (2015), which treats the informativeness of the prior as a random variable and thus also serves as a robustness check

\footnotetext{
${ }^{1}$ To ensure that our results are not driven by the ECB/Eurosystem staff projections, we also conducted the forecasting exercises without the jumping-off approach. The results are very similar and shown in the online appendix.
} 
against our choices of hyperparameters. Fourth, we estimate the baseline BVAR in levels. The results obtained via the baseline model are robust to all four alternative specifications.

The first method, entropic tilting, is based on Robertson et al. (2005). It has gained popularity in the recent years to incorporate external information into model-based forecasts, for example, see Krüger et al. (2017) for a macroeconomic application, or Altavilla et al. (2017) from the field of finance. In simple terms, by taking a possibly multivariate base distribution (e.g. the predictive distribution of a BVAR model) and a vector of moment conditions (e.g. the expected value of GDP growth at the one-quarter-ahead horizon is $0.4 \%$ ), entropic tilting provides a new, "tilted" distribution which is closest to the base distribution in the Kullback-Leibler sense, and at the same time satisfies the moment conditions. Therefore, this method allows one to add judgment to an existing forecast distribution (i) in a principled manner which is rooted in information theory, (ii) without having to re-estimate or re-specify the model that provided the base distribution, and (iii) it allows to obtain a conditional forecast that incorporates uncertainty about the conditioning values. $^{2}$ Hence, the method offers a quite general and fast post-estimation adjustment tool. Furthermore, Giacomini and Ragusa (2014) showed that if the moment condition is indeed correct, then asymptotically the entropic tilting method cannot lead to worse forecasts than using the original, base distribution when the forecasts are evaluated using the logarithmic scoring rule. ${ }^{3}$

The second method, soft conditioning, is based on Waggoner and Zha (1999). Originally, the authors aimed at producing conditional forecasts in multivariate models, for instance, to extract GDP and inflation forecasts, given a specific future path of the policy rate. In a similar spirit, the method can be used to impose a future path, of say GDP growth, where the path is based on external information. In our application, following Krüger et al. (2017), it amounts to retaining only those draws of the BVAR's predictive distribution that are within a corridor around the imposed survey expectations. Therefore, in general, and in contrast to entropic tilting, the expected value of the retained BVAR draws will not be equal to the survey expectation but merely shifted towards it. The predictions thus resemble a middle-way of the BVAR forecasts and the survey expectations. For the reader's convenience, we will generally say to "tilt" for both methods and be more precise when needed.

We take the external forecasts from the Survey of Professional Forecasters (SPF) conducted by the ECB (ECB-SPF). For evaluations of the quality of the ECB SPF forecasts, see Genre et al. (2013), Kenny et al. (2014) and Kenny et al. (2015). The overwhelming majority of panelists states that their forecasts, for horizons of one year or more, are based on judgment, or model-based with judgment adjustment, see European Central Bank (2019). Both the judgment adjustment, as well as the pooling of many opinions could be a factor in explaining the good forecasting performance of the survey. We extract the predictive mean and variance from the consensus (mean across forecasters) histogram forecasts of the fixed-horizon, one- and two-year-ahead panels for our baseline results.

Our sample of SPF forecasts ranges from 1999:Q1 to 2017:Q3. Importantly, the panelists forecast year-on-year growth rates of real GDP and HICP, and the unemployment rate one-year and two-years-ahead. The quarterly year-on-year growth is equal to the product of the quarterly

\footnotetext{
${ }^{2}$ Antolín-Díaz et al. (2019) show the equivalence of entropic tilting to linear restrictions on the mean and variance of the forecast distribution for the special case of Gaussian densities.

${ }^{3}$ More recently, Altavilla et al. (2017) suggested a test which can help researchers decide on which moments of the multivariate density they should impose external moment conditions.
} 
quarter-on-quarter growth values (and the quarterly year-on-year growth rate is approximately equal to the sum of quarterly quarter-on-quarter growth rates). ${ }^{4}$ As real GDP and the HICP enter the BVAR in 100 times logarithmic quarter-on-quarter differences, this implies that we do not impose a moment condition, for example, on the growth rate of GDP at one of the BVAR's specific horizons but rather on the product of quarterly GDP growth over several horizons.

The present paper is most closely related to Krüger et al. (2017) but differs in the following important ways. First, we tilt one-year-ahead and two-year-ahead forecasts instead of nowcasts (i.e. predictions for the current quarter) of the BVAR, due to the format of the ECB-SPF. Second, as mentioned earlier, we impose the moment condition on GDP growth or HICP inflation over several horizons of the BVAR, i.e. not on any horizon directly, which is effectively a restriction on the possible paths of these variables over several horizons. Third, we are the first to apply entropic tilting and soft conditioning using the ECB-SPF in a euro-area forecasting exercise. Another related paper by Bańbura and van Vlodrop (2018) uses euro area Consensus Economics long-run forecasts to anchor a time-varying mean of a Bayesian VAR that captures low frequency movements of the variables. Different from our work, they focus on long-run forecasts from Consensus Economics, the survey information only affects the time-varying mean and the authors do not make use of survey density forecasts. We also contribute to the growing literature using real-time euro area data (see e.g. Jarociński and Lenza (2018) or McAdam and Warne (2019) and the papers cited therein). The paper is further related to Tallman and Zaman (2018), but the authors make use of nowcast and long-run (five-year-ahead) predictions, and focus on U.S. data when applying entropic tilting. Additionally, Del Negro and Schorfheide (2013) provide evidence that incorporating survey expectations into a structural Dynamic Stochastic General Equilibrium (DSGE) model improves its forecasts.

Further, this paper relates to the literature on conditional forecasts and the forecasting exercises conducted at policy institutions as described in Angelini et al. (2019) and the references therein. Macroeconomic projections published by policy institutions, for instance by the ECB, are typically based on so called "technical assumptions", which are a set of conditional values for certain variables such as policy rates or fiscal policy developments. Bańbura et al. (2015) show how to condition on a specific path of variables for large systems, extending earlier work by Waggoner and Zha (1999). While their method updates parameter estimates given the conditioning path, the future values are assumed to be known and fix, i.e. uncertainty around the future path is not accommodated. As the SPF provides moments rather than "technical (fixed) assumptions" for future values, using moment restrictions is the obivous way to incorporate the information for improving forecasts. As noted in Andersson et al. (2010); Antolín-Díaz et al. (2019), who provide an alternative to entropic tilting for incorporating moment conditions, the moment restrictions imply a deviation from the unconditional distribution of the structural shocks of the BVAR. Assuming a correct specification of the BVAR, an improvement using the SPF would be attributed to a better understanding (or anticipation) of the panelists of which shocks are driving the economy.

The remainder of the paper is organized as follows. Section 2 introduces the BVAR and the two methods that we use to incorporate external information into the BVAR. Section 3 describes the ECB-SPF, the real-time dataset and the forecast evaluation methods. Section 4 presents the main

\footnotetext{
${ }^{4}$ We refer to $\frac{x_{t}}{x_{t-1}}$ as growth and to $\frac{x_{t}}{x_{t-1}}-1$ as growth rate.
} 
empirical results, Section 5 contains robustness exercises, and Section 6 concludes. Additional results are collected in the appendix and in the online appendix.

\section{Methodology}

\subsection{Bayesian VAR}

The baseline forecasts are obtained from a Bayesian VAR with stochastic volatility. The model is based on Clark and Ravazzolo (2015), who showed that allowing for stochastic volatility materially improves BVAR models' density forecasts. Let diag $(\cdot)$ denote the operator generating a diagonal matrix from its arguments, $\stackrel{i i d}{\sim}$ stand for independently and identically distributed, and $\mathcal{N}(\mu, \Phi)$ denote the normal distribution with mean vector $\mu$ and variance-covariance matrix $\Phi$. Let $y_{t}$ be a $(K \times 1)$ vector of variables at time $t$. Then the BVAR model is given by

$$
\begin{aligned}
y_{t} & =B_{0}+\sum_{i=1}^{p} B_{i} y_{t-i}+A^{-1} \Sigma_{t}^{\frac{1}{2}} e_{t}, \\
\Sigma_{t} & \equiv \operatorname{diag}\left(\sigma_{1, t}^{2}, \ldots, \sigma_{K, t}^{2}\right), \\
\log \left(\sigma_{k, t}^{2}\right) & =\log \left(\sigma_{k, t-1}^{2}\right)+\eta_{k, t} \text { for } k=1, \ldots, K,
\end{aligned}
$$

where $B_{0}$ is the $(K \times 1)$ vector of intercepts, $B_{i}$ s are the $(K \times K)$ coefficient matrices, $A^{-1}$ is a lower triangular matrix with ones on the diagonal, $e_{t} \stackrel{i i d}{\sim} \mathcal{N}\left(0, I_{K}\right)$ and $\left(\eta_{1, t}, \ldots, \eta_{K, t}\right)^{\prime} \stackrel{i i d}{\sim} \mathcal{N}(0, \Xi)$, where $\Xi$ is not constrained to be diagonal.

In the selection of the variables, we follow Krüger et al. (2017) and use a standard four-variable specification $(K=4)$, which includes 100 times the log-differences of real GDP and HICP, the level of the unemployment rate, and the end-of-quarter 3-month Euribor rate. The lag length is chosen according to a Bayesian Information Criterion (BIC, Schwarz, 1978) and is equal to $p=2 .^{5}$ Tables C.16 to C.19 shows that results are robust to a specification with real GDP and HICP in log-levels, instead of log-differences.

The Markov Chain Monte Carlo (MCMC) Gibbs sampler and the simulation of the predictive densities are standard and we refer to Clark and Ravazzolo (2015) for further details. For the VAR coefficients, we use a Minnesota-type prior, following the baseline specification of Carriero et al. (2015), which shrinks all coefficients of the two log-differenced variables to zero and the first own-lag coefficient of the two variables in levels to 0.8 (and all of their other coefficients to zero respectively). The only difference from Carriero et al. (2015) is that we apply somewhat more cross-variable shrinkage due to a short data sample. ${ }^{6}$ In particular, we use an overall tightness parameter of 0.2 , cross-variable tightness of 0.5 and linear lag decay. We retain each 8 th draw after a burn-in sample of 5,000 draws and, based on the oversampling algorithm of Waggoner and Zha (1999), for each of the 4,000 retained parameter draws, we draw five forecasting paths. The final number of draws that we obtain for each $y_{t+h}$, where $h=1, \ldots, 8$, is equal to 20,000, which we will refer to as MCMC draws.

As a first robustness check, to account for potential changes in coefficients, we use a time-

\footnotetext{
${ }^{5}$ In particular, at each forecast origin, we estimated the model using ordinary least squares with lag lengths from one to four. In the majority of cases the BIC suggested two lags.

${ }^{6}$ The BEAR toolbox documentation recommends very similar prior specifications (see Dieppe et al., 2016).
} 
varying parameter VAR (TVP-VAR) based on Primiceri (2005) and Del Negro and Primiceri (2015). The model allows the elements of the coefficient matrices $B_{0}$ and $B_{i}$ s to follow a random walk. The prior means and covariance matrix of the VAR coefficients are based on a VAR model estimated by ordinary least squares on a training sample of 44 pre-sample observations. We use two lags to balance the number of estimated parameters and the flexibility of the model.

As a second robustness check, we use a medium-sized 14-variable BVAR with constant coefficients and a common stochastic volatility specification based on Chan (2018). ${ }^{7}$ The usage of more predictors is motivated by the work of Bańbura et al. (2010) and Bańbura et al. (2015), who found that larger BVARs tend to forecast more accurately than smaller BVARs if sufficient shrinkage is applied in the larger models' estimation.

Additional robustness checks reported in Appendix $C$ include the estimation of the baseline BVAR but using log-levels for real GDP and the HICP and a Minnesota prior that shrinks the first own-lag coefficient to one and all other coefficients to zero. In addition, we estimate a hierarchical BVAR following Giannone et al. (2015), also in (log-)levels and using a Minnesota-type prior, a "dummy-initial-observation" and a "sum-of-coefficient" prior. In Giannone et al. (2015), the hyperparameters that control the tightness of the priors is treated as a random variable. The "dummy-initial-observation" and "sum-of-coefficient" priors provide some robustness against the potential overfitting of the BVAR because it penalizes large deviations of the model's steady state from the initial observations. Moreover, treating the hyperparameters as random variables provides some robustness to our specific choices of the hyperparameters for the previously discussed models.

\subsection{Incorporating External Forecasts into the BVAR}

The survey expectations that we use for real GDP and HICP are quarterly, one- and two-yearahead year-on-year growth rates. ${ }^{8}$ First, let us consider the one-year-ahead growth rate. Let $q_{t+4, k}$ denote the level of variable $q$ (either real GDP or HICP) at time $t+4$. The quarterly, one-year-ahead year-on-year growth, $g_{t, 4, k}$, takes the form of

$$
g_{t, 4, k}=\frac{q_{t+4, k}}{q_{t, k}}
$$

The SPF's mean prediction at time $\tau$ that we consider is then defined as $\mu_{\tau, 4, \mathrm{SPF}, k}=\mathbb{E}\left(g_{t, 4, k} \mid \Omega_{\tau}\right)$, where $\Omega_{\tau}$ denotes the information set of the SPF panelists available at time $\tau$. The SPF provides distributional forecasts in the form of histograms, which contain probabilities assigned to prespecified bins of the variable in question. We estimated $\mu_{\tau, 4, \mathrm{SPF}, k}$ non-parametrically, based on the predictive histograms. In particular, first we aggregated the individual panelists' predictions in each bin by taking the average across forecasters, then closed the bins by assuming that the lower and upper bins are of the same width as the inner ones. Finally, the mean of the SPF distribution was obtained as the probability-weighted average of the midpoints of each bin, and similarly the variance denoted by $\sigma_{\tau, 4, \mathrm{SPF}, k}^{2}$ was estimated as the probability-weighted squared deviations of the midpoints and the mean (see Giordani and Söderlind, 2003). ${ }^{9}$ The mean and variance of

\footnotetext{
${ }^{7}$ For details on the variables see Section 5.2.

${ }^{8}$ For details, see Section 3.

${ }^{9}$ As a robustness exercise, following Krüger et al. (2017), we also calculated the mean prediction based on point forecasts, and the variance as the mean squared error of past point predictions. The results are qualitatively the same
} 
the two-year-ahead growth rate were estimated analogously. Section 3 provides details on the exact timing of the ECB-SPF forecasts and the data vintages that we use. We note that due to the format of the survey and the publication lag of the variables, $\tau$ does not equal $t$. Let $r_{t+1, k}$ denote the quarterly growth rate from $t$ to $t+1$, that means

$$
r_{t+1, k}=\frac{q_{t+1, k}}{q_{t, k}}-1
$$

Real GDP and the HICP enter the BVAR in 100 times logarithmic differences which are related to the quarterly growth rates by $y_{k, t} \equiv 100\left(\log \left(q_{t, k}\right)-\log \left(q_{t-1, k}\right)\right)=100 \log \left(1+r_{t, k}\right)$. Note that $g_{t, 4, k}$ can straightforwardly be written as ${ }^{10}$

$$
\begin{aligned}
g_{t, 4, k} & =\frac{\left(1+r_{t+1, k}\right)\left(1+r_{t+2, k}\right)\left(1+r_{t+3, k}\right)\left(1+r_{t+4, k}\right) q_{t, k}}{q_{t, k}} \\
& =\left(1+r_{t+1, k}\right)\left(1+r_{t+2, k}\right)\left(1+r_{t+3, k}\right)\left(1+r_{t+4, k}\right),
\end{aligned}
$$

and similarly for the case of the two-year-ahead growth rate,

$$
g_{t, 8, k}=\prod_{j=1}^{8}\left(1+r_{t+j, k}\right) .
$$

Notice that, by imposing moment conditions on the quarterly, year-on-year forecasts of the BVAR, we effectively constrain the paths of several horizons instead of one horizon directly. As the model provides quarterly forecasts, we first transform the predictions to quarterly, year-on-year forecasts and then apply entropic tilting and soft conditioning. A potential alternative to imposing moment conditions on the year-on-year forecast would be to linearly interpolate the SPF forecast to obtain quarter-on-quarter predictions. While this approach is easily implemented for point forecasts, there is no straightforward analogue for density forecasts. In addition, we report positive "spill-over" effects, i.e. improvements for variables for which no SPF information is used.

For the unemployment rate, the ECB-SPF provides level forecasts, hence there is no need to compute growth rates.

The following paragraphs describe the two procedures for incorporating the survey information.

\subsubsection{Entropic Tilting}

This section provides a brief overview of the entropic tilting methodology, following the approach by Robertson et al. (2005) and Krüger et al. (2017), which provided a simple procedure to tilt the MCMC draws from a Bayesian VAR. It essentially amounts to re-weighting the MCMC draws such that the re-weighted draws satisfy the moment restriction(s) and the new distribution is closest to the original one.

Let $f_{t}$ denote a predictive distribution for the $(n \times 1)$ vector $x_{t+h}$, given in the form of MCMC

\footnotetext{
and are available from the authors upon request.

${ }^{10}$ Note that the quarterly, year-on-year growth rate can be approximated as the sum of quarterly, quarter-on-quarter growth rates: $\log \left(g_{t, 4, k}\right)=\log \left(\left(1+r_{t+1, k}\right)\left(1+r_{t+2, k}\right)\left(1+r_{t+3, k}\right)\left(1+r_{t+4, k}\right)\right) \approx r_{t+1, k}+r_{t+2, k}+r_{t+3, k}+r_{t+4, k}$. In the implementation, we do not use the approximation of the natural logarithm but the exact growth rate.
} 
simulation draws $\left\{x_{t+h, s}\right\}_{s=1}^{S}$, each with corresponding probability $w_{s}=1 / S .{ }^{11}$ The KullbackLeibler Information Criterion or distance (KLIC) between $f_{t}$ and another distribution $\widetilde{f}_{t}$ given by the set of probabilities $\left\{\widetilde{w}_{s}\right\}_{s=1}^{D}$ is defined as

$$
\operatorname{KLIC}\left(\widetilde{f}_{t}, f_{t}\right) \equiv \sum_{s=1}^{S} \widetilde{w}_{s} \log \left(\frac{\widetilde{w}_{s}}{w_{s}}\right)=\sum_{s=1}^{S} \widetilde{w}_{s} \log \left(S \widetilde{w}_{s}\right)
$$

Based on external information, such as survey forecasts, the researcher might want to modify the original distribution $f_{t}$, such that the new, "tilted" distribution $\widetilde{f}_{t}$ satisfies $m$ moment conditions (which $f_{t}$ does not satisfy in general). The moment conditions are collected in $(m \times 1)$ vector $\bar{g}_{\tau}$, formally $\mathbb{E}_{\widetilde{f}_{t}} g\left(x_{t+h}\right)=\bar{g}_{\tau}$, where $g: \mathbb{R}^{n} \rightarrow \mathbb{R}^{m}$, with $m \geq 1$, and $\mathbb{E}_{\widetilde{f}_{t}} g\left(x_{t+h}\right) \equiv \sum_{s=1}^{S} \widetilde{w}_{s} g\left(x_{t+h, s}\right)$ denotes the expected value of $g\left(x_{t+h}\right)$ under the distribution $\widetilde{f}_{t}$. At the same time, the researcher might not want to deviate "too much" from the original distribution $f_{t}$ in terms of KLIC.

These two objectives can be achieved through entropic tilting, which takes the form of the following optimization problem:

$$
\min _{\widetilde{f}_{t} \in H_{t}} \operatorname{KLIC}\left(\widetilde{f}_{t}, f_{t}\right) \quad \text { such that } \quad \mathbb{E}_{\widetilde{f}_{t}} g\left(x_{t+h}\right)=\bar{g}_{\tau},
$$

where $H_{t}$ is the set of feasible distributions given the MCMC draws for $f_{t}$. The solution to this problem is given by

$$
\widetilde{w}_{s}^{*}=\frac{\exp \left(\gamma^{* \prime} g\left(x_{t+h, s}\right)\right)}{\sum_{s=1}^{S} \exp \left(\gamma^{* \prime} g\left(x_{t+h, s}\right)\right)}
$$

and

$$
\gamma^{*}=\underset{\gamma}{\operatorname{argmin}} \sum_{s=1}^{S} \exp \left(\gamma^{\prime}\left(g\left(x_{t+h, s}\right)-\bar{g}_{\tau}\right)\right)
$$

where $\left\{\widetilde{w}_{s}^{*}\right\}_{S=1}^{S}$ denotes the new weights used to construct the tilted MCMC BVAR predictive density. Importantly, entropic tilting does not require any further simulation from the original BVAR distribution, as the method is just re-weighting the existing MCMC draws.

In our application, we are tilting the BVAR's raw predictive distribution towards the first and second moment forecast by the SPF, i.e. $\bar{g}_{\tau}$ contains the target distribution's the mean $\left(\mu_{\tau, 4, \mathrm{SPF}, k}\right.$ and $\left.\mu_{\tau, 8, \mathrm{SPF}, k}\right)$ and variance $\left(\sigma_{\tau, 4, \mathrm{SPF}, k}^{2}\right.$ and $\left.\sigma_{\tau, 8, \mathrm{SPF}, k}^{2}\right)$ for the appropriate variable(s).

\subsubsection{Soft Conditioning}

First suggested by Waggoner and Zha (1999), and successfully used by Krüger et al. (2017) in a setting similar to ours, we apply soft conditioning to incorporate survey information into a BVAR as an alternative to entropic tilting. Waggoner and Zha's (1999) Algorithm 2 describes how to generate a conditional forecast based on a multivariate model. In our setup, the conditioning value, i.e. the assumed path that a specific variable will follow in the future, is centered around the SPF mean prediction. To accommodate the probabilistic nature of our forecasts, we retain

\footnotetext{
${ }^{11}$ Note that $x_{t+h}$ might span several forecast horizons, and the original weights $w_{s}$ do not need to be uniform. In the empirical application, we will exploit the former.
} 
all draws that are inside an interval around that path. Similarly to Krüger et al. (2017), our soft-conditioning interval (SC-I) for variable $k$ at time $\tau$ takes the form of the Cartesian product of the intervals:

$$
\mathrm{SC}_{-} \mathrm{I}_{\tau}^{k}=\left[\mu_{\tau, 4, \mathrm{SPF}, k} \pm 1.96 \sqrt{\sigma_{\tau, 4, \mathrm{SPF}, k}^{2}}\right] \times\left[\mu_{\tau, 8, \mathrm{SPF}, k} \pm 1.96 \sqrt{\sigma_{\tau, 8, \mathrm{SPF}, k}^{2}}\right]
$$

Simply put, draws that are outside of SC-I $\mathrm{I}_{\tau}^{k}$ are discarded. In the case of soft-conditioning on several variables simultaneously (e.g. GDP growth, HICP inflation and the unemployment rate), we take the Cartesian product of the intervals and discard the MCMC draws that are outside of it, as in Krüger et al. (2017). To increase legibility of the paper we only report baseline results for soft conditioning in the main text and refer to Appendix $C$ for all additional tables.

\section{Data and Evaluation Methods}

\subsection{Survey of Professional Forecasters}

The Survey of Professional Forecasters is a quarterly survey of professional forecasters about euro area macroeconomic variables, conducted by the ECB in the middle of the first month of a quarter. It provides fixed-event (corresponding to the same calendar year in each survey round) and fixed-horizon (corresponding to a fixed number of time periods ahead) point and distribution forecasts for euro area real GDP growth, HICP inflation, and the unemployment rate. ${ }^{12}$ The fixed-horizon forecasts are available for one, two and five years ahead. Our baseline results use the one- and two-year-ahead fixed-horizon density forecasts and take the consensus, i.e. equal-weighted average of the histograms of all panelists, as explained in Section 2.2. ${ }^{13}$ The SPF sample we make use of ranges from 1999:Q1 to 2016:Q4.

Importantly, ECB-SPF forecast horizons are counted based on the latest month or quarter for which data had been released at the time of the prediction. This requires two timing decisions. First, the HICP and unemployment SPF forecasts have monthly targets, namely in the first, second, third and fourth quarters: December (of the current year), March, June, September for HICP; November (of the current year), February, May, and August for the unemployment rate. As the BVAR uses quarterly data, we treat the forecasts as having a "quarterly" target, associating the target month with the corresponding quarter. We think this timing decision could have at most minor effects on the results for two reasons: firstly, for a one-year-ahead horizon, neighboring months' forecasts would be very similar, and secondly, it would at most put the SPF predictions at a disadvantage relative to the BVAR, as the original SPF forecasts correspond to monthly year-on-year growth rates, while the BVAR is specified in quarterly terms.

Second, the timing convention of the SPF also implies that the BVAR is subject to the "ragged edge" problem, because the BVAR uses quarterly data, and GDP releases are less frequent and

\footnotetext{
${ }^{12}$ The Harmonised Index of Consumer Prices (HICP) is a price index calculated by Eurostat and the national statistical institutes in the euro area, following a common methodology (hence harmonised). The ECB's price stability objective is defined in terms of the aggregate, euro area HICP. The ECB recently added core HICP inflation to the survey questions.

${ }^{13}$ As pointed out by a discussant, the ECB-SPF point forecast is closely correlated with the ECB staff macroeconomic projections, which is published about a month before the respective SPF deadline. However, the ECB staff projections don't provide density forecasts in the form of histograms.
} 
more delayed relative to releases of HICP and the unemployment rate. ${ }^{14}$ In the SPF rounds conducted in the first, second, third, and fourth quarters, the target dates for GDP are the third (of the current year), fourth (of the current year), first (of the next year), and second (of the next year) quarters, respectively. For instance, at the time of the SPF deadline, typically two months of additional unemployment observations are available (three for HICP respectively) relative to the GDP release. We address this issue by using the jumping-off approach as developed in Faust and Wright (2009), which we describe in detail in the next subsection.

\subsection{Data for the Bayesian VAR and the Jumping-Off Method}

The real-time data on real GDP, HICP and the unemployment rate are taken from the ECB Real Time Database (Giannone et al., 2012) and our dataset ranges from 1991:Q1 to 2018:Q3. The vintages we use in the BVAR forecasts are synchronized according to the "deadline-to-reply" for SPF panelists to ensure that the BVAR and the SPF forecasts are based on the same real-time information set. Both real GDP and the unemployment rate are seasonally adjusted. We convert the latter to quarterly frequency by taking the average of the monthly observations. For HICP, the real-time data are not seasonally adjusted. Hence, we first apply the TRAMO-SEATS method to obtain seasonally adjusted data (U.S. Census Bureau (2017), implemented by Lengwiler (2017)), and then convert the monthly, seasonally adjusted HICP series to the quarterly frequency by averaging.

In addition to the real-time data, we use data from the 18th update of the Area-wide Model database (Fagan et al., 2001), that dates back to 1970:Q1 and is provided by the Euro Area Business Cycle Network. We use this dataset for two reasons. First, we obtain the (end-of-quarter) 3-month Euribor interest rate from the database, where the last observation corresponds to 2017:Q4 (the remaining three observations are downloaded from the ECB Statistical Data Warehouse). Second, we use data on all four series between 1980:Q1 and 1990:Q4 to estimate hyperparameters for the prior of the time-varying parameter BVAR in the robustness check, and to provide the scaling factors of the Minnesota-style prior covariance matrix of the BVAR coefficients.

Due to the "ragged edge" problem discussed above, we make use of the ECB's "(Broad) Macroeconomic Projection Exercise" (ECB-MPE) nowcasts, which consist of quarterly ECB and Eurosystem staff forecasts and provide, inter alia, quarter-on-quarter growth nowcasts of real GDP for the euro area. ${ }^{15}$, The macroeconomic projections are published at the beginning of the last month of a given quarter (March, June, September and December), and the nowcasts for quarter one, two, three and four are based on information up to the second half of February, May, August and November, respectively. Given this publication schedule, the MPE projections are at an information disadvantage of one month relative to the SPF. We consider this to be a minor concern for two main reasons. First, for HICP and the unemployment rate we use the same data as available to the SPF. Second, for a forecast horizon of several quarters, the effect of one month of extra information is arguably rather small.

The ECB-MPE nowcasts are plugged-in for the missing GDP data at the forecast origin (missing relative to the latest HICP and unemployment observations) which is called a jumping-

\footnotetext{
${ }^{14}$ In all discussions of the "ragged edge" problem, we omit the Euribor, as it is financial markets data and available on a weekday basis.

${ }^{15}$ In June and December, the projections are performed at the Eurosystem level, and these rounds are called Broad Macroeconomic Projection Exercises. For simplicity, we will refer to all projections rounds as ECB-MPE.
} 
off procedure (Faust and Wright, 2009). Results are robust when dealing with the "ragged edge" problem without the jumping-off procedure and are shown in Appendix C.

The BVAR is estimated in a recursive (expanding window) manner, mainly due to the relatively short initial data sample. To give an example, for the first forecast, the estimation sample of the BVAR is based on the 1999:Q1 vintage (before the release of 1998:Q4 real GDP data), i.e. the in-sample ranges from 1991:Q1 to 1998:Q3. At the time of the SPF 1999:Q1 forecasts, the HICP data for 1998:Q4 and the unemployment rate data for 1998:M10 and 1998:M11 (which we average and take as the 1998:Q4 data) were already released, but not the 1998:Q4 GDP data. Therefore, we apply the jumping-off procedure: for the forecasts the BVAR uses the realized HICP, Euribor and unemployment rate data for 1998:Q4 and we plug-in the ECB-MPE nowcast for the 1998:Q4 GDP growth value as no realization would have been available in real-time.

Consequently, the first prediction of the BVAR for $h=1$ is for 1999:Q1. In total, the out-ofsample forecast errors we evaluate range from 1999:Q1 to 2016:Q4 for the $h=1$ predictions, and from 2000:Q4 to 2018:Q3 for $h=8$ predictions, i.e. the total out-of-sample size is, therefore, $P=72$ for all horizons. Note that $h=1$ corresponds to a nowcast, i.e. predictions for the quarter of the survey round.

\subsection{Evaluation Methods}

The evaluation of the forecasts is based on vintages one quarter after the initial estimate of GDP growth, HICP inflation and the unemployment rate (all at the quarterly frequency), which resembles the timing of second-estimate vintages for the U.S. and is frequently the choice in the literature (Faust and Wright, 2009; Krüger et al., 2017). To evaluate the accuracy of the point predictions, we use the root mean squared forecasts error (RMSFE). For the evaluation of the density forecasts, we use the continuous ranked probability score (CRPS) (Gneiting et al., 2007).

Let $\widehat{y}_{k, t+h}$ denote the mean of the predictive distribution (raw BVAR, or after applying either entropic tilting or soft conditioning) of variable $k$ at horizon $h$ generated at time $t$, and $y_{k, t+h}$ stand for the corresponding realization. Then the forecast error is defined as

$$
\mathrm{FE}_{k, t, h} \equiv y_{k, t+h}-\widehat{y}_{k, t+h}
$$

while the squared forecast error is given by

$$
\mathrm{SFE}_{k, t, h} \equiv\left(y_{k, t+h}-\widehat{y}_{k, t+h}\right)^{2}
$$

and the RMSFE is defined as

$$
\mathrm{RMSFE}_{k, h} \equiv \sqrt{P^{-1} \sum_{t=1}^{P} \mathrm{SFE}_{k, t, h}} .
$$

The CRPS is a popular, strictly proper scoring rule for distribution forecasts, defined as

$$
\mathrm{CRPS}_{k, t, h} \equiv \int_{-\infty}^{\infty}\left(\widetilde{F}_{k, t, h}(y)-\mathbb{1}\left[y_{k, t+h} \leq y\right]\right)^{2} \mathrm{~d} y
$$

where $\widetilde{F}_{k, t, h}(y)$ is the empirical cumulative distribution function of the given forecasting method 
(estimated at time $t$ ) for the $k$ th variable at horizon $h$, and $\mathbb{1}[\cdot]$ is the indicator function. The average CRPS score is then given by

$$
\mathrm{CRPS}_{k, h} \equiv P^{-1} \sum_{t=1}^{P} \mathrm{CRPS}_{k, t, h}
$$

where lower values correspond to more accurate forecasts.

We note that once one has obtained the entropic tilted or soft conditioned distribution, evaluating the RMSFE or CRPS statistics does not require further simulation, as the former can be calculated by taking the (weighted) average of the original BVAR draws, while for the latter the algorithm by Hersbach (2000) can be used.

To compare model performances, we test for equal predictive ability (EPA) using the Diebold and Mariano (1995), henceforth DM test. ${ }^{16}$ Let $L_{t}\left(\widetilde{y}_{k, t+h}^{(z)}, y_{k, t+h}\right)$ denote the forecast loss - either squared forecast error defined in Equation (14) or CRPS in Equation (16) - of forecasting method $z$ at time $t$ for variable $k, h$ quarters ahead, where $\widetilde{y}_{k, t+h}^{(z)}$ is the mean of the predictive distribution (for the RMSFE evaluation) or the full predictive distribution (for the CRPS measure). The null hypothesis of equal predictive accuracy, that forecasting method $m$ is equally good as method $n$, is formally given by $\mathrm{H}_{0}: \mathbb{E}\left(L_{t}\left(\widetilde{y}_{k, t+h}^{(m)}, y_{k, t+h}\right)\right)=\mathbb{E}\left(L_{t}\left(\widetilde{y}_{k, t+h}^{(n)}, y_{k, t+h}\right)\right)$. In our analysis, method $m$ corresponds to the baseline BVAR, while method $n$ will be either entropic tilting or soft conditioning, targeting GDP growth, HICP inflation, the unemployment rate, or a combination of these. Denoting by $d_{t} \equiv L_{t}\left(\widetilde{y}_{k, t+h}^{(m)}, y_{k, t+h}\right)-L_{t}\left(\widetilde{y}_{k, t+h}^{(n)}, y_{k, t+h}\right)$ the forecast loss differential, the test statistic can be written as

$$
\mathrm{DM}_{k, h} \equiv \sqrt{P} \frac{\frac{1}{P} \sum_{t=1}^{P} d_{t}}{\sqrt{\widehat{\sigma}_{d}^{2}}}
$$

where $\widehat{\sigma}_{d}^{2}$ is an estimate of the variance of $d_{t}$, which we obtain using the Bartlett kernel of Newey and West (1987).

\section{Results}

\subsection{Main Results}

In Table 1, Panel A shows the raw BVAR RMSFE results, while Panels B to E show the ratio of the RMSFE of the tilted BVAR and the raw BVAR. The panels' description indicates the variables that were tilted. The last two columns $\left(h=4^{*}\right.$ and $\left.h=8^{*}\right)$ contain results for the one-year-ahead and two-year-ahead growth rates of GDP and HICP, corresponding to the original SPF survey question. Values below one indicate improvements over the raw BVAR. Boldface numbers imply a superior performance of the tilted BVAR. One, two or three asterisks imply rejection of equal predictive ability at the $10 \%, 5 \%$ and $1 \%$ significance level - that is, statistically significant differences in predictive performance. As Panel B shows, tilting towards the SPF GDP predictions yields improvements of the short- as well as medium-term forecasts, which are statistically significant for the first two horizons, as well as the year-on-year predictions. The improvement for the first two horizons is an interesting result because the tilting is applied to quarterly, year-on-year growth

\footnotetext{
${ }^{16}$ For a discussion of asymptotic validity of the DM EPA test in this setup, see Krüger et al. (2017).
} 
rates, i.e. longer horizons. In terms of economic size, the tilting procedure leads to a reduction of the RMSFE of up to $20 \%$. The spillover effects to "other-than-targeted" variables are relevant in size and statistically significant for the unemployment rate and the Euribor. The improvements for tilting HICP, in Panel C, are sizeable in economic terms and, while not statistically significant for HICP itself, show sizeable and statistically significant spillover effects to the Euribor. Tilting unemployment, shown in Panel D, yields improvements for the unemployment rate itself and a slight decrease in predictive accuracy for GDP for longer horizons and HICP. Finally, tilting all three variable jointly in Panel E shows sizeable improvements for all four variables. The appendix additionally shows results of the multi-horizon forecast comparison test of Quaedvlieg (2019), which controls the family-wise error rate of the multiple hypothesis tests displayed in Table 1 and the tables thereafter.

The economic size of the results, implies that the tilted BVAR improves the quarter-on-quarter forecasts of GDP approximately by about 0.1 percentage points, and the year-on-year forecasts approximately by about 0.3 percentage points. ${ }^{17}$

Table C.3 shows the results for the density forecast evaluation. Improvements due to tilting are comparable, and even stronger in terms of economic size and statistical significance, relative to the RMSFE results.

Figure 1 shows the results for tilting all three variables jointly — the plot corresponds to Panel E of Tables 1 and C.3.

Table 3 shows the absolute (Panel A) and relative (Panels B to E) RMSFE statistics for the case of soft conditioning. Results are similar to entropic tilting, but the reductions in the RMSFEs tend to be somewhat smaller.

In terms of density forecasts, Table C.5 demonstrates that incorporating SPF information on GDP growth through soft conditioning considerably improves the raw BVAR forecasts (Panel B), and additionally tilting inflation and unemployment towards SPF forecasts results in some additional gains (Panel E).

\footnotetext{
${ }^{17}$ These numbers are calculated via the product of the RMSFE ratio and the RMSFE of the BVAR, assuming a constant forecast error of the BVAR.
} 
Table 1: Entropic tilting versus BVAR forecasts: RMSFE

\begin{tabular}{|c|c|c|c|c|c|c|c|c|c|c|}
\hline \multicolumn{2}{|c|}{ Forecast horizon $h=1$} & $h=2$ & $h=3$ & $h=4$ & $h=5$ & $h=6$ & $h=7$ & $h=8$ & $h=4^{*}$ & $h=8^{*}$ \\
\hline & \multicolumn{10}{|c|}{ Panel A. BVAR } \\
\hline GDP & 0.55 & 0.59 & 0.59 & 0.58 & 0.58 & 0.58 & 0.57 & 0.57 & 1.68 & 1.93 \\
\hline $\mathrm{HICP}$ & 0.26 & 0.27 & 0.29 & 0.30 & 0.31 & 0.31 & 0.31 & 0.31 & 0.92 & 1.07 \\
\hline Unemployment & 0.29 & 0.46 & 0.65 & 0.83 & 1.00 & 1.16 & 1.30 & 1.43 & $N / A$ & $N / A$ \\
\hline \multirow[t]{2}{*}{ Euribor } & 0.35 & 0.66 & 0.94 & 1.20 & 1.44 & 1.64 & 1.82 & 1.97 & $N / A$ & $N / A$ \\
\hline & \multicolumn{10}{|c|}{ Panel B. Tilting GDP } \\
\hline GDP & $0.80^{* *}$ & $0.86^{*}$ & 0.91 & 0.98 & 1.00 & 1.00 & 1.00 & 1.02 & $0.81^{* *}$ & 1.00 \\
\hline $\mathrm{HICP}$ & 1.00 & 1.00 & 1.01 & 1.01 & 1.02 & 1.02 & 1.01 & 1.01 & 1.01 & 1.02 \\
\hline Unemployment & 0.96 & $0.93^{*}$ & $0.92^{*}$ & 0.93 & 0.93 & 0.94 & 0.95 & 0.96 & $N / A$ & $N / A$ \\
\hline \multirow[t]{2}{*}{ Euribor } & 0.98 & $0.95^{* *}$ & $0.93^{* *}$ & $0.94^{* *}$ & $0.95^{*}$ & 0.97 & 0.99 & 0.99 & $N / A$ & $N / A$ \\
\hline & \multicolumn{10}{|c|}{ Panel C. Tilting HICP } \\
\hline GDP & 0.98 & 0.98 & 0.98 & 0.99 & 0.99 & 1.00 & 1.00 & 1.00 & 0.98 & 0.99 \\
\hline HICP & 0.93 & 0.98 & 0.93 & 0.93 & 0.92 & 0.92 & 0.91 & 0.91 & 0.91 & 0.88 \\
\hline Unemployment & 0.98 & 0.98 & 0.98 & 0.98 & 0.99 & 0.99 & 1.00 & 1.01 & $N / A$ & $N / A$ \\
\hline \multirow[t]{2}{*}{ Euribor } & 0.96 & 0.92 & $0.88^{*}$ & $0.86^{*}$ & $0.85^{*}$ & $0.84^{*}$ & $0.84^{* *}$ & $0.85^{* *}$ & $N / A$ & $N / A$ \\
\hline & \multicolumn{10}{|c|}{ Panel D. Tilting Unemployment } \\
\hline GDP & 0.99 & 0.97 & 0.99 & 0.99 & $1.03^{* *}$ & $1.10^{*}$ & 1.07 & $1.04^{* * *}$ & 0.96 & 1.00 \\
\hline $\mathrm{HICP}$ & $1.03^{* *}$ & $1.02^{* *}$ & 1.02 & 1.02 & 1.02 & 1.03 & 1.02 & 1.03 & $1.03^{* *}$ & 1.04 \\
\hline Unemployment & 0.93 & 0.88 & $0.86^{* *}$ & $0.86^{* *}$ & $0.87^{* * *}$ & $0.88^{* * *}$ & $0.88^{* * *}$ & $0.89^{* * *}$ & $N / A$ & $N / A$ \\
\hline \multirow[t]{2}{*}{ Euribor } & 1.04 & 0.98 & 0.97 & 0.99 & 0.99 & 0.98 & 0.98 & 0.98 & $N / A$ & $N / A$ \\
\hline & \multicolumn{10}{|c|}{ Panel E. Tilting GDP, HICP and Unemployment } \\
\hline GDP & $0.80^{* *}$ & $0.86^{* *}$ & 0.93 & 1.00 & 1.00 & 1.01 & 1.01 & $1.06^{* *}$ & $0.82^{* *}$ & 0.99 \\
\hline $\mathrm{HICP}$ & 0.93 & 0.96 & 0.93 & 0.95 & 0.92 & 0.91 & 0.92 & 0.94 & 0.91 & 0.88 \\
\hline Unemployment & 0.91 & $0.86^{*}$ & $0.84^{* *}$ & $0.86^{* *}$ & $0.87^{* * *}$ & $0.88^{* * *}$ & $0.89^{* * *}$ & $0.90^{* * *}$ & $N / A$ & $N / A$ \\
\hline Euribor & 0.93 & $0.84^{* *}$ & $0.83^{* *}$ & $0.83^{* *}$ & $0.84^{* *}$ & $0.85^{* *}$ & $0.86^{* *}$ & $0.86^{* *}$ & $N / A$ & $N / A$ \\
\hline
\end{tabular}

Note: Panel A displays the raw BVAR RMSFE. Panels B to E show the ratios of the RMSFE of the entropic tilted BVAR to the raw BVAR. Numbers in bold imply that the tilted BVAR improves over the raw BVAR. Asterisks ${ }^{*},{ }^{* *}$ and ${ }^{* * *}$ imply statistical significance of a two-sided Diebold and Mariano (1995) test of equal predictive ability at the 10\%, $5 \%, 1 \%$ level. The variance is estimated using Bartlett kernel weights with bandwidth equal to the forecast horizon $h$. The evaluation sample size is $P=72$ quarters. The columns $h=4^{*}$ and $h=8^{*}$ show the results for the one- and two-year-ahead, year-on-year predictions, respectively. 
Table 2: Entropic tilting versus BVAR forecasts: CRPS

\begin{tabular}{|c|c|c|c|c|c|c|c|c|c|c|}
\hline \multicolumn{2}{|c|}{ Forecast horizon $h=1$} & $h=2$ & $h=3$ & $h=4$ & $h=5$ & $h=6$ & $h=7$ & $h=8$ & $h=4^{*}$ & $h=8^{*}$ \\
\hline & \multicolumn{10}{|c|}{ Panel A. BVAR } \\
\hline GDP & 0.27 & 0.29 & 0.29 & 0.29 & 0.29 & 0.29 & 0.28 & 0.28 & 0.90 & 1.08 \\
\hline HICP & 0.15 & 0.16 & 0.17 & 0.17 & 0.18 & 0.18 & 0.18 & 0.18 & 0.54 & 0.63 \\
\hline Unemployment & 0.18 & 0.28 & 0.40 & 0.54 & 0.66 & 0.78 & 0.89 & 0.99 & $N / A$ & $N / A$ \\
\hline \multirow[t]{2}{*}{ Euribor } & 0.17 & 0.32 & 0.49 & 0.65 & 0.81 & 0.95 & 1.07 & 1.18 & $N / A$ & $N / A$ \\
\hline & \multicolumn{10}{|c|}{ Panel B. Tilting GDP } \\
\hline GDP & $0.79^{* * *}$ & $0.81^{*}$ & 0.86 & 0.93 & 0.95 & 0.96 & 0.97 & 1.00 & $0.76^{* * *}$ & 0.97 \\
\hline HICP & 1.00 & 1.01 & 1.02 & 1.02 & 1.02 & 1.02 & 1.01 & 1.01 & 1.02 & 1.02 \\
\hline Unemployment & 0.97 & 0.95 & 0.93 & 0.93 & 0.93 & 0.94 & 0.95 & 0.96 & $N / A$ & $N / A$ \\
\hline \multirow[t]{2}{*}{ Euribor } & 0.98 & $0.95^{*}$ & $0.93^{* *}$ & $0.93^{* *}$ & $0.94^{* *}$ & $0.95^{* *}$ & 0.96 & 0.96 & $N / A$ & $N / A$ \\
\hline & \multicolumn{10}{|c|}{ Panel C. Tilting HICP } \\
\hline GDP & 0.98 & 0.98 & 0.98 & 0.99 & 0.99 & 0.99 & 0.99 & 1.00 & 0.98 & 0.99 \\
\hline HICP & $0.93^{*}$ & 0.97 & 0.92 & 0.92 & 0.92 & 0.91 & 0.90 & 0.90 & 0.93 & 0.89 \\
\hline Unemployment & 0.98 & 0.98 & 0.98 & 0.99 & 0.99 & 1.00 & 1.01 & 1.01 & $N / A$ & $N / A$ \\
\hline \multirow[t]{2}{*}{ Euribor } & 0.98 & 0.95 & 0.91 & 0.89 & 0.87 & $0.86^{*}$ & $0.86^{*}$ & $0.86^{*}$ & $N / A$ & $N / A$ \\
\hline & \multicolumn{10}{|c|}{ Panel D. Tilting Unemployment } \\
\hline GDP & 1.01 & 1.01 & 1.02 & 1.00 & $1.05^{*}$ & $1.08^{*}$ & 1.10 & $1.06^{* *}$ & 0.96 & 0.99 \\
\hline HICP & $1.03^{*}$ & $1.02^{* *}$ & 1.01 & 1.02 & 1.01 & 1.02 & 1.01 & 1.02 & $1.04^{*}$ & 1.01 \\
\hline Unemployment & $0.91^{* *}$ & $0.88^{* *}$ & $0.83^{* * *}$ & $0.81^{* * *}$ & $0.81^{* * *}$ & $0.80^{* * *}$ & $0.81^{* * *}$ & $0.83^{* * *}$ & $N / A$ & $N / A$ \\
\hline \multirow[t]{2}{*}{ Euribor } & 1.06 & 1.00 & 0.98 & 0.99 & 0.98 & 0.96 & 0.96 & 0.97 & $N / A$ & $N / A$ \\
\hline & \multicolumn{10}{|c|}{ Panel E. Tilting GDP, HICP and Unemployment } \\
\hline GDP & $0.79^{* * *}$ & $0.82^{*}$ & 0.89 & 0.95 & 0.95 & 0.97 & 1.00 & 1.04 & $0.76^{* * *}$ & 0.96 \\
\hline $\mathrm{HICP}$ & 0.93 & 0.96 & 0.94 & 0.95 & 0.93 & 0.90 & 0.91 & 0.92 & 0.93 & 0.89 \\
\hline Unemployment & $0.89^{* * *}$ & $0.85^{* *}$ & $0.80^{* * *}$ & $0.79^{* * *}$ & $0.79^{* * *}$ & $0.79^{* * *}$ & $0.81^{* * *}$ & $0.83^{* * *}$ & $N / A$ & $N / A$ \\
\hline Euribor & 0.98 & 0.89 & $0.86^{*}$ & $0.85^{*}$ & $0.84^{* *}$ & $0.85^{* *}$ & $0.84^{* *}$ & $0.84^{* *}$ & $N / A$ & $N / A$ \\
\hline
\end{tabular}

Note: Panel A displays the raw BVAR CRPS. Panels B to E show the ratios of the CRPS of the entropic tilted BVAR to the raw BVAR. Numbers in bold imply that the tilted BVAR improves over the raw BVAR. Asterisks *, ** and *** imply statistical significance of a two-sided Diebold and Mariano (1995) test of equal predictive ability at the 10\%, $5 \%, 1 \%$ level. The variance is estimated using Bartlett kernel weights with bandwidth equal to the forecast horizon $h$. The evaluation sample size is $P=72$ quarters. The columns $h=4^{*}$ and $h=8^{*}$ show the results for the one- and two-year-ahead, year-on-year predictions, respectively. 
Figure 1: Tilting towards the SPF forecasts jointly

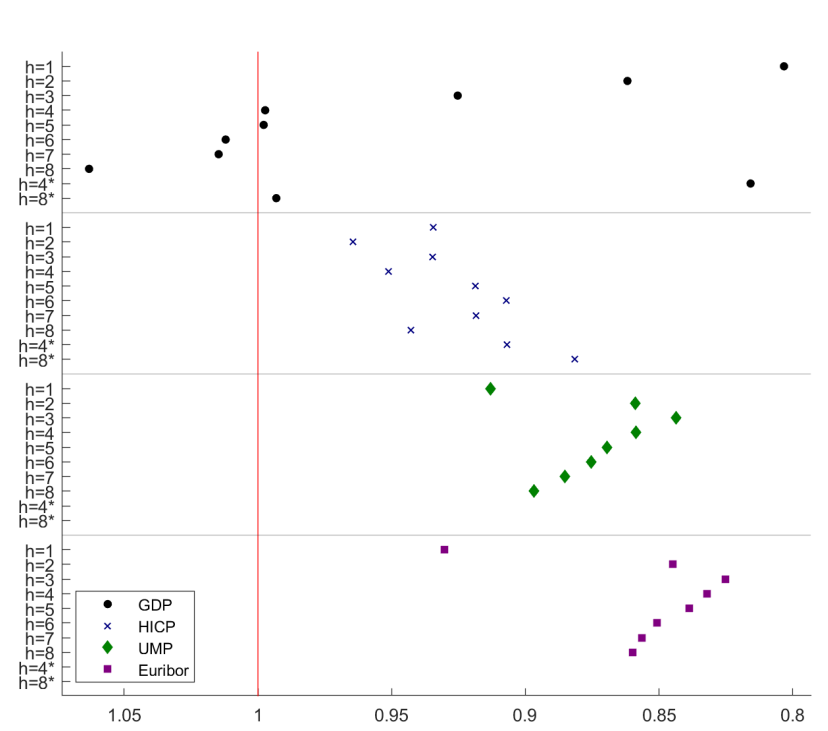

(a) RMSFE Ratio

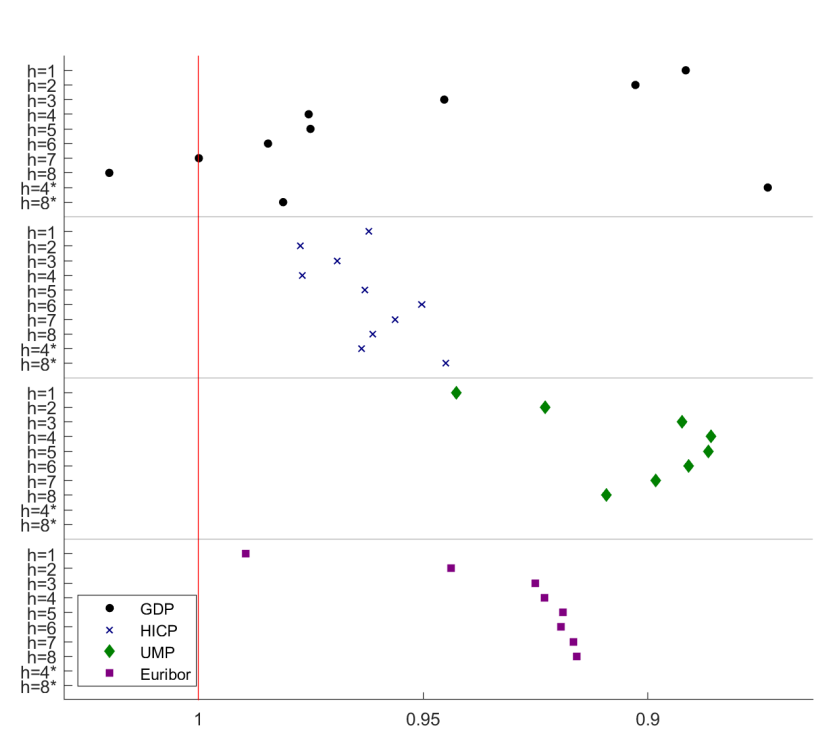

(c) CRPS Ratio

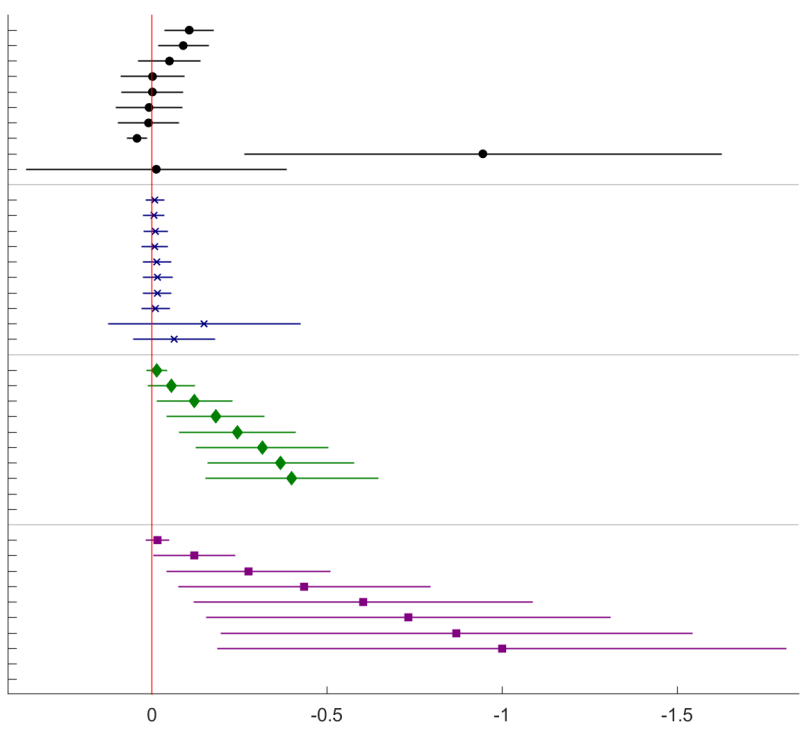

(b) RMSFE Difference

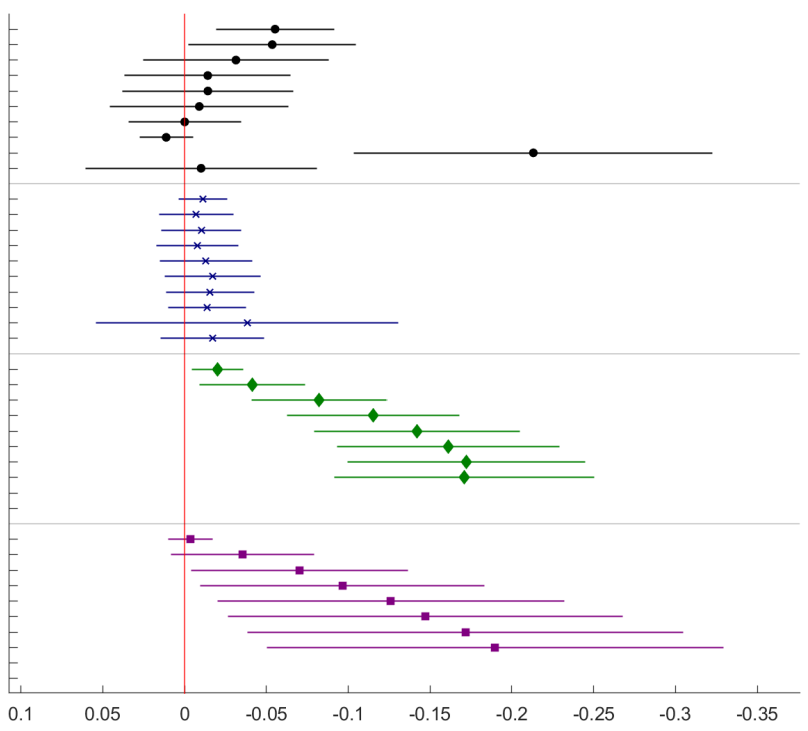

(d) CRPS Difference

Note: The figure shows results when tilting the BVAR predictions jointly towards the one- and two-year-ahead SPF forecasts of real GDP growth, HICP inflation and the unemployment rate. Panel (a) and Panel (c) display the RMSFE ratio of the tilted BVAR and the raw BVAR. A number smaller than one implies that the tilted BVAR outperforms the raw BVAR. Panel (b) and Panel (d) display the RMSFE loss differential of the tilted BVAR minus the raw BVAR. A number smaller than zero implies that the tilted BVAR outperforms the raw BVAR. The axis label $h$ denotes the forecast horizon. The horizontal lines centered around a marker denote $90 \%$ confidence intervals, for which the variance is estimated using Bartlett kernel weights with bandwidth equal to the forecast horizon $h$. To improve legibility, all RSMFE loss differentials of the Euribor have been normalized by the respective value for $h=8 *$. 
Table 3: Soft Conditioning versus BVAR forecasts: RMSFE

\begin{tabular}{|c|c|c|c|c|c|c|c|c|c|c|}
\hline \multicolumn{2}{|c|}{ Forecast horizon $h=1$} & $h=2$ & $h=3$ & $h=4$ & $h=5$ & $h=6$ & $h=7$ & $h=8$ & $h=4^{*}$ & $h=8^{*}$ \\
\hline & \multicolumn{10}{|c|}{ Panel A. BVAR } \\
\hline GDP & 0.55 & 0.59 & 0.59 & 0.58 & 0.58 & 0.58 & 0.57 & 0.57 & 1.68 & 1.93 \\
\hline HICP & 0.26 & 0.27 & 0.29 & 0.30 & 0.31 & 0.31 & 0.31 & 0.31 & 0.92 & 1.07 \\
\hline Unemployment & 0.29 & 0.46 & 0.65 & 0.83 & 1.00 & 1.16 & 1.30 & 1.43 & $N / A$ & $N / A$ \\
\hline \multirow[t]{2}{*}{ Euribor } & 0.35 & 0.66 & 0.94 & 1.20 & 1.44 & 1.64 & 1.82 & 1.97 & $N / A$ & $N / A$ \\
\hline & \multicolumn{10}{|c|}{ Panel B. Soft Conditioning GDP } \\
\hline GDP & $0.88^{* *}$ & $0.91^{* * *}$ & 0.93 & 0.97 & 0.99 & 0.99 & 1.00 & 1.01 & $0.89^{* *}$ & 0.98 \\
\hline HICP & 1.00 & 1.00 & 1.00 & 1.00 & 1.01 & 1.01 & 1.01 & 1.01 & 1.00 & 1.01 \\
\hline Unemployment & 0.98 & $0.96^{*}$ & $0.96^{* *}$ & $0.96^{*}$ & 0.96 & 0.97 & 0.97 & 0.98 & $N / A$ & $N / A$ \\
\hline \multirow[t]{2}{*}{ Euribor } & $0.99^{*}$ & $0.97^{* *}$ & $0.96^{* *}$ & $0.96^{* * *}$ & $0.97^{* * *}$ & $0.98^{*}$ & 0.99 & 0.99 & $N / A$ & $N / A$ \\
\hline & \multicolumn{10}{|c|}{ Panel C. Soft Conditioning HICP } \\
\hline GDP & 0.99 & 0.99 & 0.99 & 1.00 & 1.00 & 1.00 & 1.00 & $1.00^{*}$ & 0.99 & 1.00 \\
\hline HICP & $0.97^{*}$ & 0.98 & 0.95 & 0.94 & 0.94 & 0.93 & $0.92^{*}$ & $0.93^{*}$ & 0.94 & $0.90^{*}$ \\
\hline Unemployment & 0.99 & 0.99 & 0.99 & 0.99 & 0.99 & 1.00 & 1.00 & 1.00 & $N / A$ & $N / A$ \\
\hline \multirow[t]{2}{*}{ Euribor } & 0.99 & $0.96^{*}$ & $0.94^{*}$ & $0.93^{*}$ & $0.92^{*}$ & $0.92^{* *}$ & $0.92^{* *}$ & $0.93^{* *}$ & $N / A$ & $N / A$ \\
\hline & \multicolumn{10}{|c|}{ Panel D. Soft Conditioning Unemployment } \\
\hline GDP & $0.99^{*}$ & $0.99^{*}$ & 1.00 & 1.00 & 1.00 & 1.00 & 1.00 & 1.01 & 1.00 & 1.00 \\
\hline HICP & 1.00 & 1.00 & 1.00 & 1.00 & 1.01 & 1.01 & 1.01 & 1.01 & 1.00 & 1.02 \\
\hline Unemployment & 0.98 & 0.97 & $0.96^{*}$ & $0.97^{*}$ & $0.97^{*}$ & $0.97^{*}$ & $0.97^{*}$ & $0.97^{*}$ & $N / A$ & $N / A$ \\
\hline \multirow[t]{2}{*}{ Euribor } & 1.01 & 1.01 & 1.01 & 1.01 & 1.01 & $1.01^{*}$ & $1.01^{*}$ & $1.01^{*}$ & $N / A$ & $N / A$ \\
\hline & \multicolumn{10}{|c|}{ Panel E. Soft Conditioning GDP, HICP and Unemployment } \\
\hline GDP & $0.87^{* *}$ & $0.90^{* *}$ & 0.93 & 0.97 & 0.99 & 0.99 & 1.00 & 1.01 & $0.88^{* *}$ & 0.98 \\
\hline $\mathrm{HICP}$ & 0.97 & 0.98 & 0.96 & 0.95 & 0.94 & 0.94 & $0.93^{*}$ & $0.93^{* *}$ & 0.95 & $0.91^{*}$ \\
\hline Unemployment & $0.96^{*}$ & $0.94^{*}$ & $0.93^{*}$ & $0.94^{*}$ & $0.94^{*}$ & 0.95 & 0.96 & 0.97 & $N / A$ & $N / A$ \\
\hline Euribor & 0.98 & $0.94^{* *}$ & $0.91^{* *}$ & $0.90^{* *}$ & $0.91^{* *}$ & $0.91^{* *}$ & $0.92^{* *}$ & $0.93^{* *}$ & $N / A$ & $N / A$ \\
\hline
\end{tabular}

Note: Panel A displays the raw BVAR RMSFE. Panels B to E show the ratios of the RMSFE of the soft conditioned BVAR to the raw BVAR. Numbers in bold imply that the soft conditioned BVAR improves over the raw BVAR. Asterisks *, ** and ${ }^{* * *}$ imply statistical significance of a two-sided Diebold and Mariano (1995) test of equal predictive ability at the $10 \%, 5 \%, 1 \%$ level. The variance is estimated using Bartlett kernel weights with bandwidth equal to the forecast horizon $h$. The evaluation sample size is $P=72$ quarters. The columns $h=4^{*}$ and $h=8^{*}$ show the results for the oneand two-year-ahead, year-on-year predictions, respectively. 
Table 4: Soft Conditioning versus BVAR forecasts: CRPS

\begin{tabular}{|c|c|c|c|c|c|c|c|c|c|c|}
\hline \multicolumn{2}{|c|}{ Forecast horizon $h=1$} & $h=2$ & $h=3$ & $h=4$ & $h=5$ & $h=6$ & $h=7$ & $h=8$ & $h=4^{*}$ & $h=8^{*}$ \\
\hline & \multicolumn{10}{|c|}{ Panel A. BVAR } \\
\hline GDP & 0.27 & 0.29 & 0.29 & 0.29 & 0.29 & 0.29 & 0.28 & 0.28 & 0.90 & 1.08 \\
\hline HICP & 0.15 & 0.16 & 0.17 & 0.17 & 0.18 & 0.18 & 0.18 & 0.18 & 0.54 & 0.63 \\
\hline Unemployment & 0.18 & 0.28 & 0.40 & 0.54 & 0.66 & 0.78 & 0.89 & 0.99 & $N / A$ & $N / A$ \\
\hline \multirow[t]{2}{*}{ Euribor } & 0.17 & 0.32 & 0.49 & 0.65 & 0.81 & 0.95 & 1.07 & 1.18 & $N / A$ & $N / A$ \\
\hline & \multicolumn{10}{|c|}{ Panel B. Soft Conditioning GDP } \\
\hline GDP & $0.87^{* * *}$ & $0.88^{* *}$ & $0.91^{* *}$ & 0.95 & 0.97 & 0.97 & 0.98 & 1.00 & $0.89^{*}$ & 1.01 \\
\hline $\mathrm{HICP}$ & 1.00 & 1.00 & 1.01 & 1.01 & 1.01 & 1.01 & 1.01 & 1.01 & 1.01 & 1.02 \\
\hline Unemployment & 0.99 & 0.98 & 0.98 & 0.98 & 0.98 & 0.98 & 0.99 & 0.99 & $N / A$ & $N / A$ \\
\hline \multirow[t]{2}{*}{ Euribor } & $0.98^{* * *}$ & $0.97^{* * *}$ & $0.96^{* * *}$ & $0.96^{* * *}$ & $0.96^{* * *}$ & $0.97^{* *}$ & 0.97 & 0.97 & $N / A$ & $N / A$ \\
\hline & \multicolumn{10}{|c|}{ Panel C. Soft Conditioning HICP } \\
\hline GDP & 0.99 & 0.99 & 0.99 & 0.99 & $0.99^{*}$ & $0.99^{* *}$ & $1.00^{* *}$ & 1.00 & 0.99 & 1.00 \\
\hline HICP & 0.97 & 0.98 & 0.96 & 0.95 & 0.94 & 0.94 & $0.93^{*}$ & $0.93^{*}$ & 0.97 & 0.93 \\
\hline Unemployment & 1.00 & 0.99 & 1.00 & 1.00 & 1.00 & 1.01 & $1.01^{* *}$ & $1.02^{* *}$ & $N / A$ & $N / A$ \\
\hline \multirow[t]{2}{*}{ Euribor } & 0.99 & 0.97 & 0.95 & $0.94^{*}$ & $0.93^{* *}$ & $0.93^{*}$ & $0.93^{* *}$ & $0.93^{* *}$ & $N / A$ & $N / A$ \\
\hline & \multicolumn{10}{|c|}{ Panel D. Soft Conditioning Unemployment } \\
\hline GDP & $0.99^{*}$ & 0.99 & 1.00 & 1.00 & $0.99^{*}$ & 1.00 & 1.00 & 1.00 & 1.00 & 1.00 \\
\hline HICP & 1.00 & $1.00^{*}$ & 1.00 & 1.01 & 1.01 & 1.01 & 1.01 & 1.01 & $1.01^{*}$ & 1.02 \\
\hline Unemployment & 0.99 & 0.98 & 0.97 & 0.97 & 0.98 & 0.98 & 0.99 & 0.99 & $N / A$ & $N / A$ \\
\hline \multirow[t]{2}{*}{ Euribor } & 1.01 & 1.01 & 1.01 & 1.01 & 1.01 & 1.01 & 1.01 & 1.01 & $N / A$ & $N / A$ \\
\hline & \multicolumn{10}{|c|}{ Panel E. Soft Conditioning GDP, HICP and Unemployment } \\
\hline GDP & $0.87^{* * *}$ & $0.88^{* *}$ & $0.91^{* * *}$ & 0.95 & 0.97 & 0.97 & 0.98 & 1.01 & $0.90^{* *}$ & 1.01 \\
\hline HICP & 0.98 & 0.99 & 0.97 & 0.96 & 0.96 & 0.95 & 0.95 & 0.95 & 0.99 & 0.96 \\
\hline Unemployment & 0.98 & 0.96 & 0.96 & 0.96 & 0.97 & 0.98 & 0.99 & 1.00 & $N / A$ & $N / A$ \\
\hline Euribor & 0.98 & $0.95^{* *}$ & $0.92^{* *}$ & $0.91^{* *}$ & $0.91^{* *}$ & $0.91^{* * *}$ & $0.92^{* *}$ & $0.92^{* *}$ & $N / A$ & $N / A$ \\
\hline
\end{tabular}

Note: Panel A displays the raw BVAR CRPS. Panels B to E show the ratios of the CRPS of the soft conditioned BVAR to the raw BVAR. Numbers in bold imply that the soft conditioned BVAR improves over the raw BVAR. Asterisks *, ** and ${ }^{* * *}$ imply statistical significance of a two-sided Diebold and Mariano (1995) test of equal predictive ability at the $10 \%, 5 \%, 1 \%$ level. The variance is estimated using Bartlett kernel weights with bandwidth equal to the forecast horizon $h$. The evaluation sample size is $P=72$ quarters. The columns $h=4^{*}$ and $h=8^{*}$ show the results for the one- and two-year-ahead, year-on-year predictions, respectively. 
The histograms in Panel (a) of Figure 2 show an example of the two-quarter-ahead raw BVAR predictive distribution and its entropically tilted counterpart for real GDP growth based on the 2013:Q3 SPF round (that is, the BVAR's latest observation corresponds to 2013:Q2, and the target date is 2013:Q4). The tilted distribution is shifted to the left, less dispersed and exhibits a higher probability mass around the realization. Panel (b) is the soft conditioning counterpart of Panel (a), showing a very similar effect: the BVAR distribution is shifted closer to the actual realization, and its dispersion is reduced.

Figure 2: The effect of entropic tilting and soft conditioning on two-quarter-ahead (2013:Q4 over 2013:Q3) GDP growth forecast in the 2013:Q3 SPF round

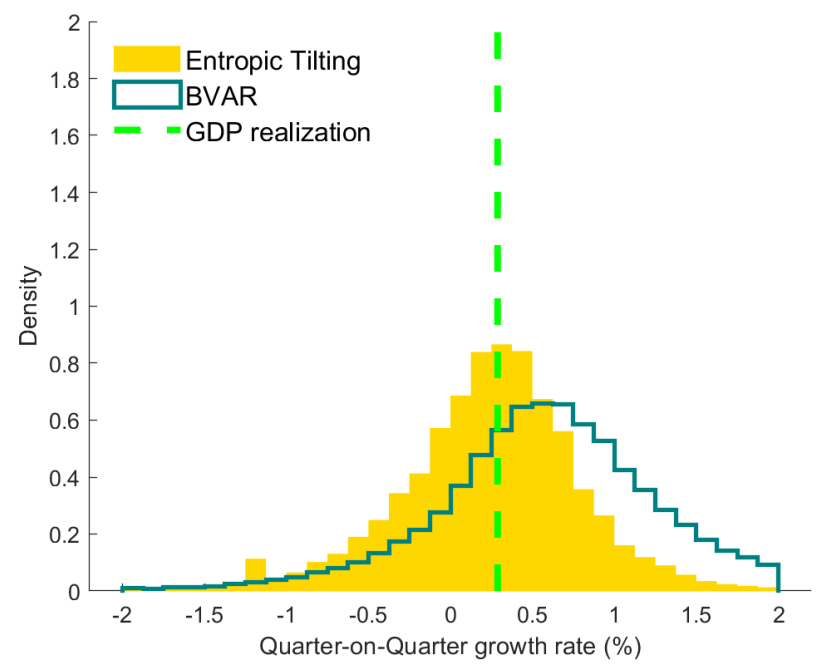

(a) Entropic tilting

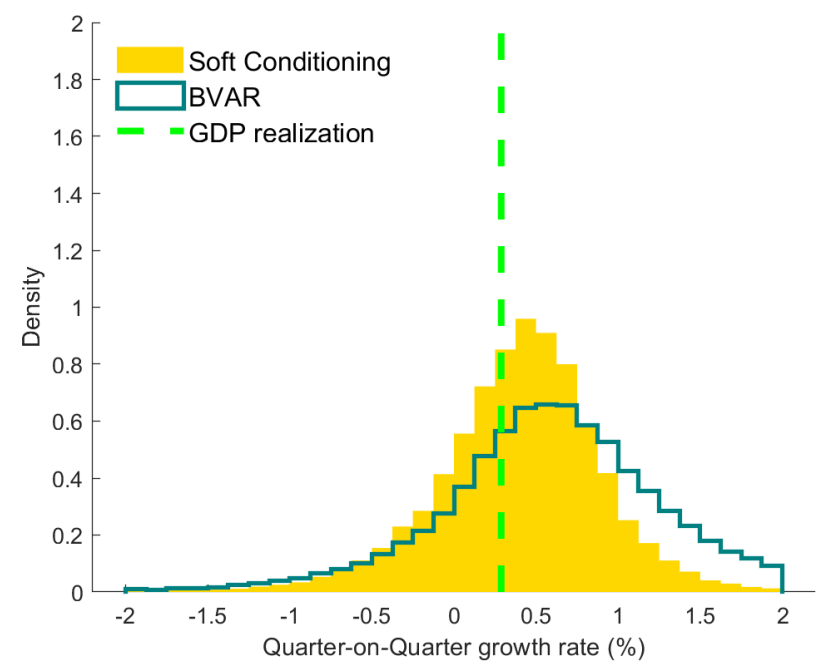

(b) Soft conditioning

Note: Panel (a) displays the histograms of the predictive distribution of GDP growth (2013:Q4 over 2013:Q3) produced by the BVAR and the entropic tilted BVAR (tilted to the SPF GDP growth forecast), at the 2013:Q3 SPF round. The BVAR's latest observation corresponds to 2013:Q2, and its forecast horizon is $h=2$ quarters. Panel (b) shows analogous results for soft conditioning. The vertical dashed line indicates the realization.

\subsection{Tilting Towards the Mean Only}

The previous results are based on jointly tilting towards the SPF mean and variance prediction. To understand better where the main gains in forecasting performance come from, Table 5 shows results when tilting only towards the SPF mean while leaving the second moment of the predictive density unchanged. ${ }^{18}$ Comparing the results to table Table C.3, we see that the main part of the gain comes from tilting towards the SPF mean.

\footnotetext{
${ }^{18}$ We only display results of the CRPS here because the RMSFE results are by definition unchanged.
} 
Table 5: Tilting towards SPF mean and BVAR Variance: CRPS

\begin{tabular}{|c|c|c|c|c|c|c|c|c|c|c|}
\hline \multirow[t]{2}{*}{ Forecast horizon } & $h=1$ & $h=2$ & $h=3$ & $h=4$ & $h=5$ & $h=6$ & $h=7$ & $h=8$ & $h=4^{*}$ & $h=8^{*}$ \\
\hline & \multicolumn{10}{|c|}{ Panel A. Tilting GDP } \\
\hline GDP & 0.27 & 0.29 & 0.29 & 0.29 & 0.29 & 0.29 & 0.28 & 0.28 & 0.90 & 1.08 \\
\hline HICP & 0.15 & 0.16 & 0.17 & 0.17 & 0.18 & 0.18 & 0.18 & 0.18 & 0.54 & 0.63 \\
\hline Unemployment & 0.18 & 0.28 & 0.40 & 0.54 & 0.66 & 0.78 & 0.89 & 0.99 & $N / A$ & $N / A$ \\
\hline \multirow[t]{2}{*}{ Euribor } & 0.17 & 0.32 & 0.49 & 0.65 & 0.81 & 0.95 & 1.07 & 1.18 & $N / A$ & $N / A$ \\
\hline & \multicolumn{10}{|c|}{ Panel B. Tilting GDP } \\
\hline GDP & $0.82^{* * *}$ & $0.83^{* *}$ & 0.88 & 0.97 & 1.00 & 1.00 & 1.01 & 1.01 & $0.76^{* *}$ & 0.99 \\
\hline HICP & 1.00 & 1.01 & 1.01 & 1.01 & 1.00 & 1.00 & 1.00 & 0.99 & 1.01 & 0.99 \\
\hline Unemployment & 0.96 & 0.93 & 0.92 & 0.93 & 0.94 & 0.95 & 0.96 & 0.96 & $N / A$ & $N / A$ \\
\hline \multirow[t]{2}{*}{ Euribor } & 1.00 & 0.98 & 0.97 & $0.96^{*}$ & $0.96^{* *}$ & $0.96^{* *}$ & $0.97^{*}$ & 0.97 & $N / A$ & $N / A$ \\
\hline & \multicolumn{10}{|c|}{ Panel C. Tilting HICP } \\
\hline GDP & 0.99 & 0.99 & 0.99 & 1.00 & 0.99 & 1.00 & 1.00 & 1.00 & 0.98 & 0.99 \\
\hline HICP & 0.95 & 0.98 & 0.93 & 0.93 & $0.93^{*}$ & $0.91^{* *}$ & $0.91^{* *}$ & $0.93^{* *}$ & 0.93 & $0.88^{* *}$ \\
\hline Unemployment & 0.99 & 1.00 & 1.01 & $1.02^{* *}$ & $1.02^{* *}$ & $1.03^{* * *}$ & $1.03^{* * *}$ & $1.03^{* * *}$ & $N / A$ & $N / A$ \\
\hline \multirow[t]{2}{*}{ Euribor } & 0.99 & 0.97 & 0.95 & 0.94 & 0.94 & $0.94^{*}$ & $0.94^{*}$ & $0.95^{*}$ & $N / A$ & $N / A$ \\
\hline & \multicolumn{10}{|c|}{ Panel D. Tilting Unemployment } \\
\hline GDP & $0.94^{* * *}$ & 0.95 & 0.97 & $0.97^{*}$ & 1.01 & 1.01 & $1.04^{* *}$ & $1.04^{* *}$ & $0.92^{* * *}$ & 0.99 \\
\hline HICP & 1.01 & $1.02^{*}$ & $1.02^{*}$ & 1.02 & 1.01 & 1.01 & 1.00 & 1.01 & $1.03^{* *}$ & 1.01 \\
\hline Unemployment & $0.92^{*}$ & $0.88^{* *}$ & $0.83^{* * *}$ & $0.82^{* * *}$ & $0.82^{* * *}$ & $0.83^{* * *}$ & $0.84^{* * *}$ & $0.86^{* * *}$ & $N / A$ & $N / A$ \\
\hline \multirow[t]{2}{*}{ Euribor } & 1.02 & 0.99 & 0.97 & 0.97 & 0.96 & 0.95 & 0.96 & 0.96 & $N / A$ & $N / A$ \\
\hline & \multicolumn{10}{|c|}{ Panel E. Tilting GDP, HICP and Unemployment } \\
\hline GDP & $0.79^{* * *}$ & $0.84^{*}$ & 0.90 & 0.99 & 1.02 & 1.01 & $1.04^{*}$ & 1.08 & $0.76^{* *}$ & 0.99 \\
\hline HICP & 0.95 & 0.98 & 0.94 & 0.93 & 0.93 & 0.93 & 0.94 & 0.94 & 0.94 & 0.90 \\
\hline Unemployment & $0.90^{*}$ & $0.86^{* *}$ & $0.82^{* * *}$ & $0.82^{* * *}$ & $0.82^{* * *}$ & $0.84^{* * *}$ & $0.86^{* * *}$ & $0.87^{* *}$ & $N / A$ & $N / A$ \\
\hline Euribor & 0.98 & 0.92 & $0.90^{*}$ & $0.91^{*}$ & $0.90^{* *}$ & $0.90^{* *}$ & $0.91^{* *}$ & $0.91^{*}$ & $N / A$ & $N / A$ \\
\hline
\end{tabular}

Note: Panel A displays the raw BVAR CRPS. Panels B to E show the ratios of the CRPS of the entropic tilted BVAR to the raw BVAR. Numbers in bold imply that the tilted BVAR improves over the raw BVAR. Asterisks ${ }^{*},{ }^{* *}$ and ${ }^{* * *}$ imply statistical significance of a two-sided Diebold and Mariano (1995) test of equal predictive ability at the $10 \%, 5 \%, 1 \%$ level. The variance is estimated using Bartlett kernel weights with bandwidth equal to the forecast horizon $h$. The evaluation sample size is $P=72$ quarters. The columns $h=4^{*}$ and $h=8^{*}$ show the results for the one- and two-year-ahead, year-on-year predictions, respectively. 


\subsection{Structural Change and Survey Forecasts}

After the Great Recession, the euro area experienced lower real growth and inflation rates than in the pre-crisis years. In what follows, we adopt the CEPR Euro Area Business Cycle Dating Committee's chronology, and take 2008:Q1 as the peak of the business cycle before the Great Recession, and 2009:Q2 as the trough of the crisis. ${ }^{19}$

To investigate the potential bias of the BVAR's and the tilted predictions, we regressed the forecast error in Equation (13) on a constant, and conducted a $t$-test on the coefficient, testing the null hypothesis of a zero coefficient (or unbiasedness). Table 6 shows the bias of the forecast error of the raw BVAR and the tilted BVAR before the Great Recession with the last realized forecast error dated 2008:Q1, and after the Great Recession with forecast origins 2009:Q3 to 2017:Q3. Taking into account the small subsample sizes due to the split, $37-h$ and 30 data points before and after the crisis, respectively, we consider the results of the hypothesis tests as being suggestive rather than decisive evidence. However, Panel A that prior to the financial crisis, the BVAR exhibits no bias for growth, a small positive and statistically significant bias for inflation in the short run, i.e. it underestimated inflation (recall that in Equation (13) we defined the forecast error as the realization minus the point forecast) and a somewhat larger but non-significant bias for the interest rate. However, as Panel D demonstrates, after the crisis, the bias is negative for GDP growth, statistically significant, and considerably larger in size - the BVAR overestimates real GDP growth. After tilting the model forecast, as Panel F displays, the statistical significance of the bias in the real GDP growth predictions disappears for all horizons but $h=8$ and $h=8^{*}$. Importantly, at horizons used directly in the tilting procedure, $(h=1, \ldots, 7)$, the tilted BVAR exhibits virtually no bias, similarly to the one-year-ahead year-on-year predictions $\left(h=4^{*}\right)$. While the raw BVAR is slow to adjust to the structural break, the SPF adapts quickly in real-time. Importantly, in a dynamic system, such as a VAR, a structural break in one of the variables can cause a bias in other variables, as multiple-step-ahead forecasts are conditioned on the draws of the other variables. In fact, Panels $\mathrm{E}$ and $\mathrm{F}$ show that the spillover effects from correcting the bias in real GDP forecasts reduce the size of the bias of the unemployment rate and the Euribor forecasts.

Figure 3 reflects some the results of the previous table by showing the evolution of the point forecasts of the three competing models and the corresponding realizations. The BVAR tends to overestimate real GDP growth after the financial crisis. It also somewhat underestimates inflation before the recession. Figure 4 shows the CRPS loss differential of the raw BVAR and the tilted BVAR. Negative values imply a superior performance of the tilted BVAR. The performance improvement using tilting is similar to what we can observe inFigure 3. Using judgment-based information can help to deal with this type of structural changes and is easily implemented via entropic tilting or soft conditioning.

A potential, model-based remedy to the deteriorating effect of structural changes on the forecasting performance is allowing for time-varying parameters. Therefore, we investigate the performance of a time-varying parameter BVAR with stochastic volatility (TVP-VAR) in the next section.

\footnotetext{
${ }^{19}$ The full chronology and the analyses are available at https://cepr.org/content/ euro-area-business-cycle-dating-committee.
} 
Table 6: Forecast bias — before and after the Great Recession

\begin{tabular}{|c|c|c|c|c|c|c|c|c|c|c|}
\hline \multirow[t]{2}{*}{ Forecast horizon } & $h=1$ & $h=2$ & $h=3$ & $h=4$ & $h=5$ & $h=6$ & $h=7$ & $h=8$ & $h=4^{*}$ & $h=8^{*}$ \\
\hline & \multicolumn{10}{|c|}{ Panel A. BVAR - Before Great Recession } \\
\hline GDP & -0.01 & -0.02 & -0.01 & -0.02 & -0.03 & -0.04 & -0.04 & -0.04 & -0.28 & -0.28 \\
\hline HICP & $0.08^{* *}$ & $0.10^{* *}$ & $0.10^{*}$ & 0.10 & 0.10 & 0.10 & 0.10 & 0.09 & 0.38 & 0.28 \\
\hline Unemployment & -0.05 & -0.07 & -0.10 & -0.13 & -0.14 & -0.15 & -0.15 & -0.14 & $N / A$ & $N / A$ \\
\hline \multirow[t]{2}{*}{ Euribor } & -0.04 & -0.05 & -0.06 & -0.08 & -0.14 & -0.21 & -0.33 & -0.47 & $N / A$ & $N / A$ \\
\hline & \multicolumn{10}{|c|}{ Panel B. Tilting GDP One-Year-Ahead - Before Great Recession } \\
\hline GDP & -0.07 & -0.07 & -0.06 & -0.03 & -0.04 & -0.05 & -0.06 & -0.06 & -0.37 & -0.34 \\
\hline HICP & $0.08^{* *}$ & $0.09^{* *}$ & $0.10^{*}$ & 0.10 & 0.10 & 0.10 & 0.10 & 0.09 & 0.38 & 0.28 \\
\hline Unemployment & -0.04 & -0.06 & -0.08 & -0.11 & -0.13 & -0.14 & -0.14 & -0.13 & $N / A$ & $N / A$ \\
\hline \multirow[t]{2}{*}{ Euribor } & -0.05 & -0.08 & -0.10 & -0.13 & -0.18 & -0.25 & -0.36 & -0.51 & $N / A$ & $N / A$ \\
\hline & \multicolumn{10}{|c|}{ Panel C. Tilting GDP One-Year and Two-Years-Ahead - Before Great Recession } \\
\hline GDP & -0.06 & -0.07 & -0.06 & -0.13 & -0.14 & -0.15 & -0.15 & -0.09 & -0.37 & -0.72 \\
\hline HICP & $0.08^{* *}$ & $0.10^{* *}$ & $0.09^{*}$ & 0.10 & 0.10 & 0.10 & 0.10 & 0.09 & 0.38 & 0.27 \\
\hline Unemployment & -0.05 & -0.07 & -0.10 & -0.13 & -0.14 & -0.14 & -0.12 & -0.11 & $N / A$ & $N / A$ \\
\hline \multirow[t]{2}{*}{ Euribor } & -0.02 & -0.02 & -0.01 & -0.03 & -0.09 & -0.18 & -0.31 & -0.46 & $N / A$ & $N / A$ \\
\hline & \multicolumn{10}{|c|}{ Panel D. BVAR - After Great Recession } \\
\hline GDP & $-0.27^{* *}$ & $-0.33^{* *}$ & $-0.31^{* *}$ & $-0.29^{* *}$ & $-0.30^{* *}$ & $-0.29^{* *}$ & $-0.27^{*}$ & $-0.29^{* *}$ & -0.81 & -1.09 \\
\hline HICP & 0.01 & 0.02 & -0.00 & -0.01 & -0.02 & -0.03 & -0.05 & -0.06 & 0.04 & -0.14 \\
\hline Unemployment & 0.04 & 0.09 & 0.14 & 0.19 & 0.23 & 0.25 & 0.27 & 0.27 & $N / A$ & $N / A$ \\
\hline \multirow[t]{2}{*}{ Euribor } & 0.01 & -0.05 & -0.16 & $-0.27^{*}$ & $-0.39^{* *}$ & $-0.51^{* * *}$ & $-0.64^{* * *}$ & $-0.76^{* * *}$ & $N / A$ & $N / A$ \\
\hline & \multicolumn{10}{|c|}{ Panel E. Tilting GDP One-Year-Ahead - After Great Recession } \\
\hline GDP & -0.06 & -0.07 & -0.07 & $-0.25^{*}$ & $-0.30^{* *}$ & $-0.29^{* *}$ & $-0.28^{*}$ & $-0.29^{* *}$ & -0.09 & -1.06 \\
\hline HICP & 0.01 & 0.03 & 0.02 & 0.01 & -0.00 & -0.01 & -0.02 & -0.04 & 0.10 & -0.06 \\
\hline Unemployment & 0.00 & 0.00 & -0.00 & 0.02 & 0.04 & 0.07 & 0.09 & 0.10 & $N / A$ & $N / A$ \\
\hline \multirow[t]{2}{*}{ Euribor } & 0.06 & 0.10 & 0.12 & 0.07 & -0.02 & -0.14 & -0.27 & $-0.41^{*}$ & $N / A$ & $N / A$ \\
\hline & \multicolumn{10}{|c|}{ Panel F. Tilting GDP One-Year and Two-Years-Ahead - After Great Recession } \\
\hline GDP & -0.06 & -0.08 & $-0.05^{\circ}$ & -0.10 & -0.12 & -0.11 & -0.11 & $-0.26^{*}$ & -0.09 & -0.36 \\
\hline $\mathrm{HICP}$ & 0.01 & 0.03 & 0.02 & 0.01 & 0.01 & 0.00 & -0.01 & -0.02 & 0.10 & 0.01 \\
\hline Unemployment & 0.01 & 0.02 & 0.02 & 0.02 & 0.01 & -0.01 & -0.03 & -0.04 & $N / A$ & $N / A$ \\
\hline Euribor & 0.05 & 0.08 & 0.09 & 0.08 & 0.07 & 0.03 & -0.01 & -0.09 & $N / A$ & $N / A$ \\
\hline
\end{tabular}

Note: Panels A to F display the means of the forecast errors of the BVAR and tilted BVAR, before and after the Great Recession. Asterisks ${ }^{*},{ }^{* *}$ and ${ }^{* * *}$ imply statistical significance of a two-sided $t$-test of unbiasedness at the $10 \%, 5 \%, 1 \%$ level. The variance is estimated using Bartlett kernel weights with bandwidth equal to the forecast horizon $h$. The evaluation sample size is $37-h$ quarters in Panels A to C, and 30 quarters in Panels D to F. The columns labeled $h=4^{*}$ and $h=8^{*}$ show the results for the one-year-ahead and two-year-ahead, year-on-year predictions, respectively. 
Figure 3: Mean predictions with tilting

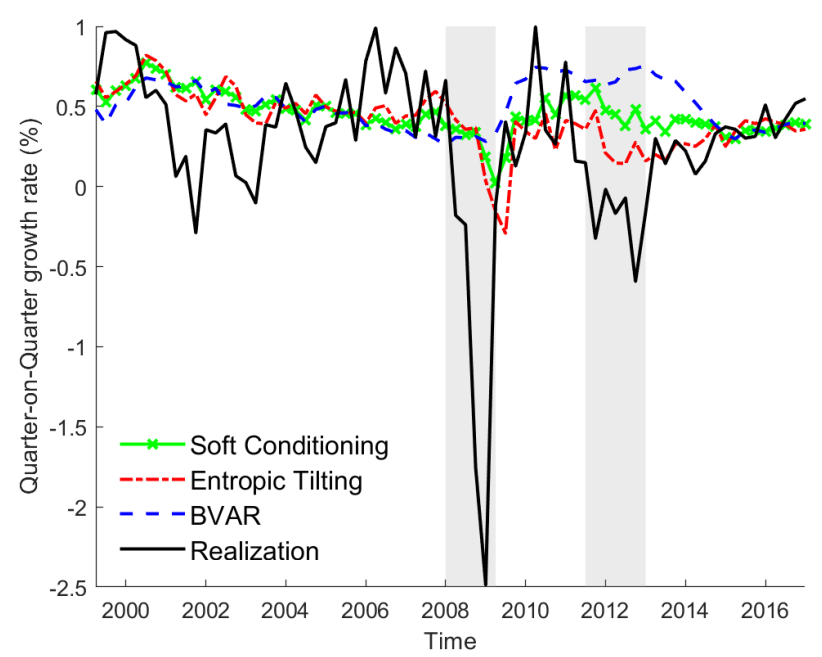

(a) GDP, $h=2$

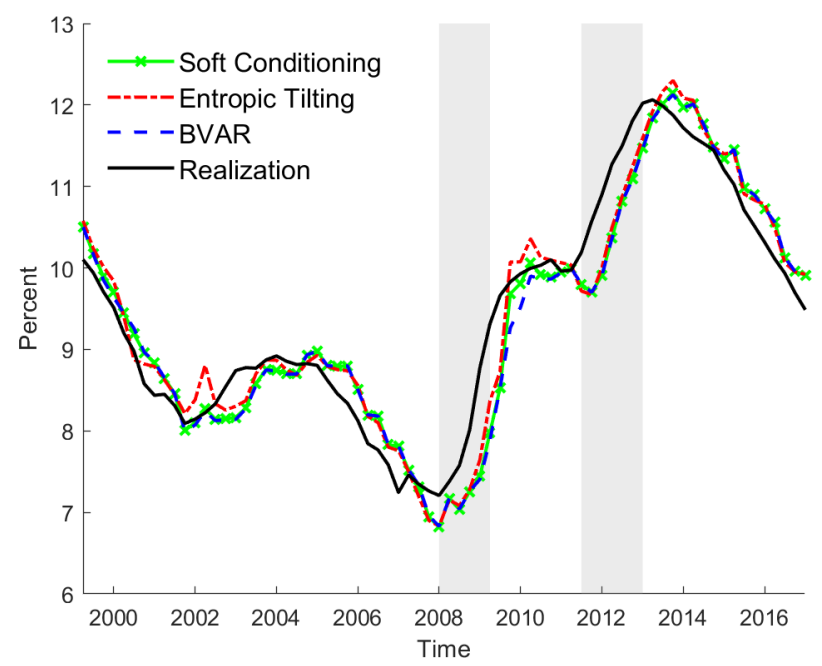

(c) Unemployment rate, $h=2$

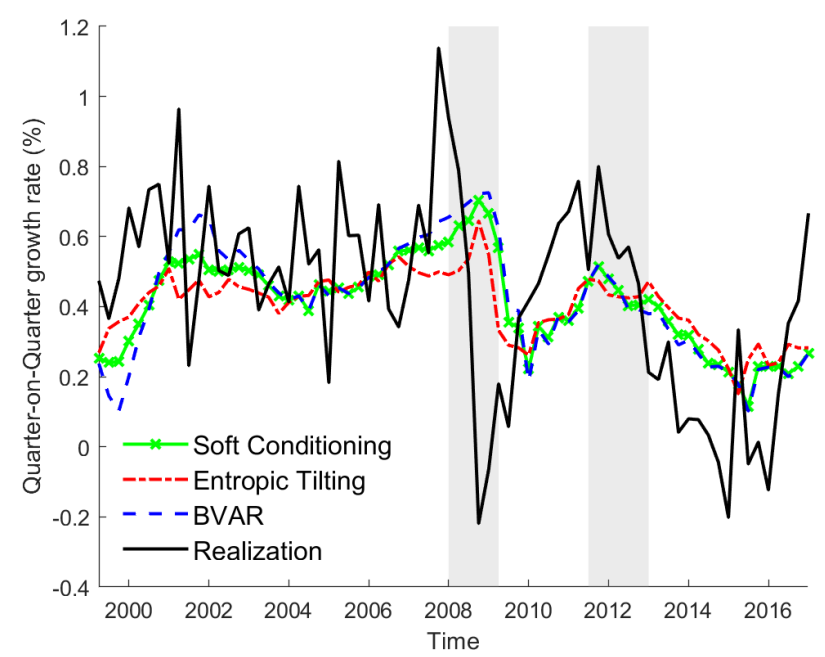

(b) HICP, $h=2$

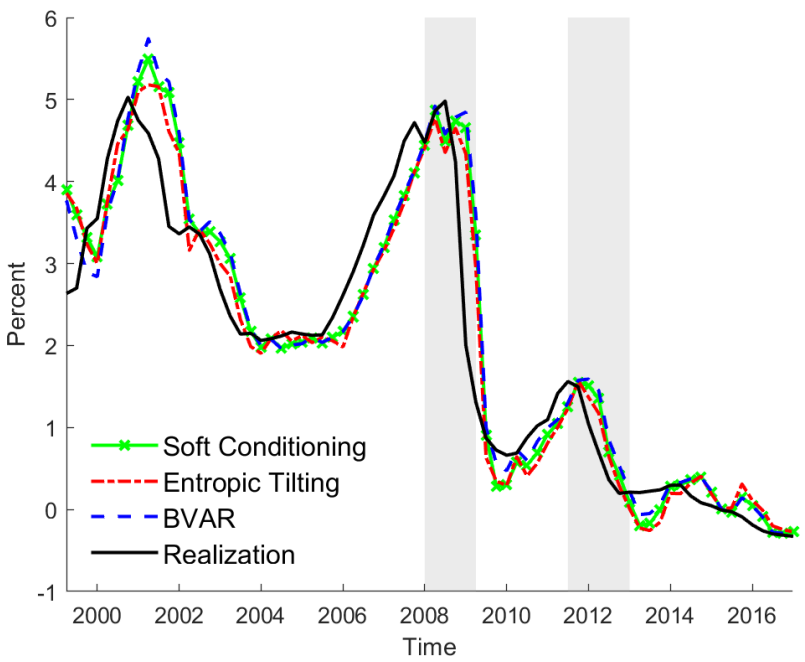

(d) Euribor, $h=2$

Note: The figures show mean predictions of the raw BVAR, the entropic tilted BVAR and soft conditioning. Time on the horizontal axis corresponds to the target dates, $h$ denotes the forecasting horizon. The entropic tilting and soft conditioning results are based on the specification that incorporate SPF GDP forecasts for Panel (a), the SPF HICP forecasts for Panel (b), the SPF unemployment rate forecasts for Panel (c) and the joint tilting for Panel (d). 
Figure 4: CRPS Differences

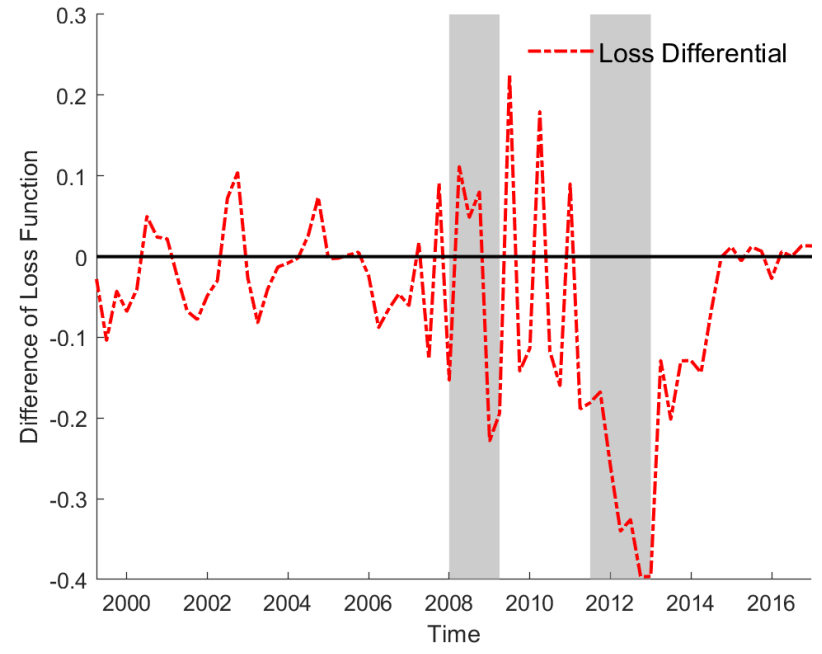

(a) GDP, $h=2$

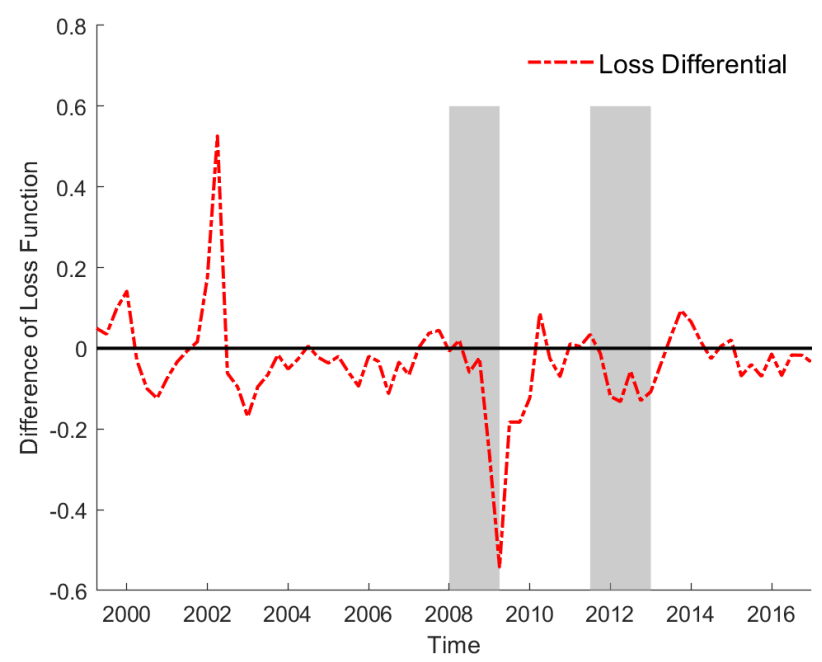

(c) Unemployment rate, $h=2$

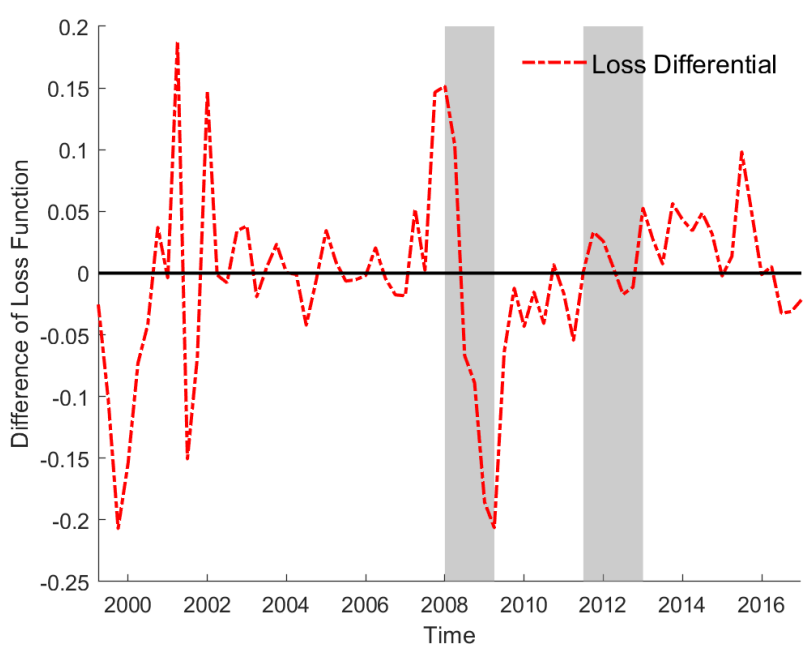

(b) HICP, $h=2$

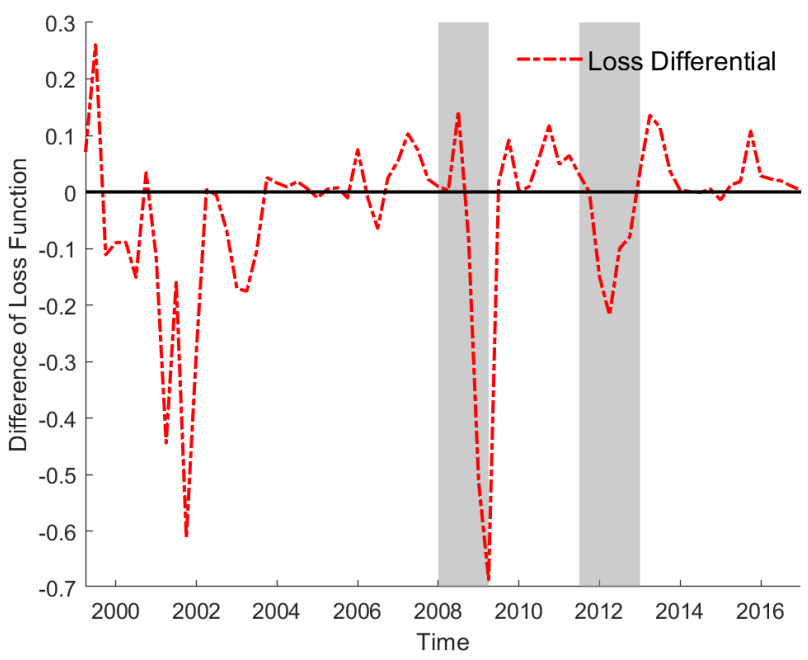

(d) Euribor, $h=2$

Note: The figures show the differences of the CRPS of the raw BVAR and the entropic tilted BVAR. Time on the horizontal axis corresponds to the target dates, $h$ denotes the forecasting horizon. A negative value implies a smaller loss for the tilted BVAR. The entropic tilting results are based on the specification that incorporate SPF GDP forecasts for Panel (a), the SPF HICP forecasts for Panel (b), the SPF unemployment rate forecasts for Panel (c) and the joint tilting for Panel (d). 


\section{Robustness Results}

\subsection{Time-varying parameter VAR}

Using U.S. data, D'Agostino et al. (2013) showed that allowing for time-varying parameters, as in Primiceri (2005), can improve the forecasting performance of a BVAR. The time variation might help mitigate the effect of persistently lower growth rates after the financial crisis on the forecasting performance. We ensured that the resulting VAR is stable by using the multimove sampler of Koop and Potter (2011) that allows for inequality restrictions. Without the inequality restrictions of smaller than unit eigenvalues of the companion form matrices of the time-varying autoregressive coefficients, the predictive densities exhibited very large dispersions which deteriorated the TVP-VARs forecasting performance.

Table 7: TVP-VAR: forecast bias — before and after the Great Recession

\begin{tabular}{|c|c|c|c|c|c|c|c|c|c|c|}
\hline \multirow{2}{*}{\multicolumn{2}{|c|}{ Forecast horizon $h=1$}} & $h=2$ & $h=3$ & $h=4$ & $h=5$ & $h=6$ & $h=7$ & $h=8$ & $h=4^{*}$ & $h=8^{*}$ \\
\hline & & \multicolumn{9}{|c|}{ Panel A. TVP-VAR - Before Great Recession } \\
\hline GDP & -0.10 & -0.15 & -0.15 & -0.16 & -0.17 & -0.17 & -0.18 & -0.17 & -0.60 & -0.87 \\
\hline HICP & 0.01 & -0.01 & -0.05 & -0.06 & -0.09 & -0.11 & -0.13 & -0.15 & -0.15 & -0.62 \\
\hline Unemployment & -0.03 & -0.04 & -0.06 & -0.07 & -0.08 & -0.09 & -0.08 & -0.09 & $N / A$ & $N / A$ \\
\hline \multirow[t]{2}{*}{ Euribor } & $-0.19^{* *}$ & $-0.46^{* *}$ & $-0.76^{* *}$ & $-1.06^{* *}$ & $-1.37^{* *}$ & $-1.66^{* *}$ & $-1.97^{* *}$ & $-2.27^{* *}$ & $N / A$ & $N / A$ \\
\hline & \multicolumn{10}{|c|}{ Panel B. Tilting GDP One-Year and Two-Years-Ahead - Before Great Recession } \\
\hline GDP & -0.04 & -0.08 & -0.08 & -0.13 & -0.14 & -0.15 & -0.16 & -0.17 & -0.37 & -0.72 \\
\hline HICP & -0.01 & -0.03 & -0.07 & -0.09 & -0.11 & $-0.13^{*}$ & $-0.15^{*}$ & $-0.17^{*}$ & -0.24 & -0.71 \\
\hline Unemployment & -0.04 & -0.06 & -0.09 & -0.13 & -0.16 & -0.18 & -0.19 & -0.21 & $N / A$ & $N / A$ \\
\hline \multirow[t]{2}{*}{ Euribor } & $-0.21^{* *}$ & $-0.50^{* *}$ & $-0.81^{* *}$ & $-1.11^{* *}$ & $-1.41^{* *}$ & $-1.71^{* *}$ & $-2.01^{* *}$ & $-2.31^{* *}$ & $N / A$ & $N / A$ \\
\hline & \multicolumn{10}{|c|}{ Panel C. TVP-VAR - After Great Recession } \\
\hline GDP & -0.14 & -0.17 & -0.17 & -0.19 & -0.22 & -0.23 & -0.23 & $-0.25^{*}$ & -0.38 & -0.82 \\
\hline HICP & 0.06 & 0.09 & 0.09 & 0.09 & 0.10 & 0.09 & 0.09 & 0.07 & 0.36 & 0.36 \\
\hline Unemployment & -0.01 & 0.00 & 0.02 & 0.05 & 0.08 & 0.11 & 0.14 & 0.17 & $N / A$ & $N / A$ \\
\hline \multirow[t]{2}{*}{ Euribor } & 0.05 & 0.08 & 0.06 & 0.02 & -0.06 & -0.16 & -0.28 & -0.43 & $N / A$ & $N / A$ \\
\hline & \multicolumn{10}{|c|}{ Panel D. Tilting GDP One-Year and Two-Years-Ahead - After Great Recession } \\
\hline GDP & -0.06 & -0.07 & -0.06 & -0.08 & -0.11 & -0.12 & -0.13 & $-0.20^{*}$ & -0.09 & -0.36 \\
\hline HICP & 0.06 & 0.10 & 0.11 & 0.12 & 0.13 & 0.13 & 0.13 & 0.12 & 0.42 & 0.52 \\
\hline Unemployment & -0.02 & -0.03 & -0.05 & -0.06 & -0.07 & -0.08 & -0.09 & -0.09 & $N / A$ & $N / A$ \\
\hline Euribor & 0.07 & 0.14 & 0.19 & 0.22 & 0.24 & 0.24 & 0.21 & 0.16 & $N / A$ & $N / A$ \\
\hline
\end{tabular}

Note: Panels A to D display forecast error means of the TVP-VAR and tilted TVP-VAR, before and after the Great Recession. Asterisks ${ }^{*},{ }^{* *}$ and ${ }^{* *}$ imply statistical significance of a two-sided $t$-test of unbiasedness at the $10 \%, 5 \%, 1 \%$ level. The variance is estimated using Bartlett kernel weights with bandwidth equal to the forecast horizon $h$. The evaluation sample size is $37-h$ quarters in Panels A and B, and 30 quarters in Panels C and D. The columns labeled $h=4^{*} h=8^{*}$ show the results for the one- and two-year-ahead, year-on-year predictions, respectively.

Table 7 displays the results of the forecast unbiasedness test based on the TVP-VAR predictions. As conjectured, the TVP-VAR exhibits a considerably smaller deviation from a zero mean for all four variables, and the bias is only statistically significant for the case of $h=8$ after the Great Recession (Panels C and D). Figure 5 shows the forecast comparison of the TVP-VAR to the tilted TVP-VAR in terms of RMSFE and CRPS, for the case of a joint tilting towards all three variables. Again, tilting improves the forecast performance in almost all cases, except for short-horizons of the interest rate forecast. Tables C.1 and C.2 in the appendix display the full set of results, including when tilting variables individually. The gains from individual tilting are particularly large for the case of GDP but reductions in the RMSFE and the CRPS economically 
and also statistically significant in almost all cases. Appendix $C$ shows additional results for soft conditioning, which are qualitatively the same.

Figure 5: Results when tilting the TVP-VAR towards the one- and-two-year-ahead SPF forecast

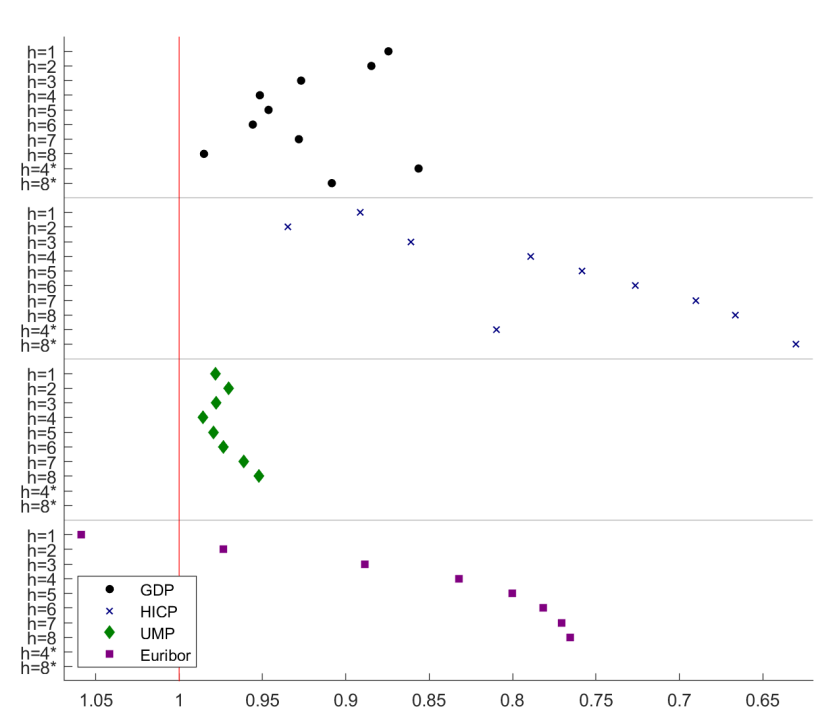

(a) RMSFE Ratio

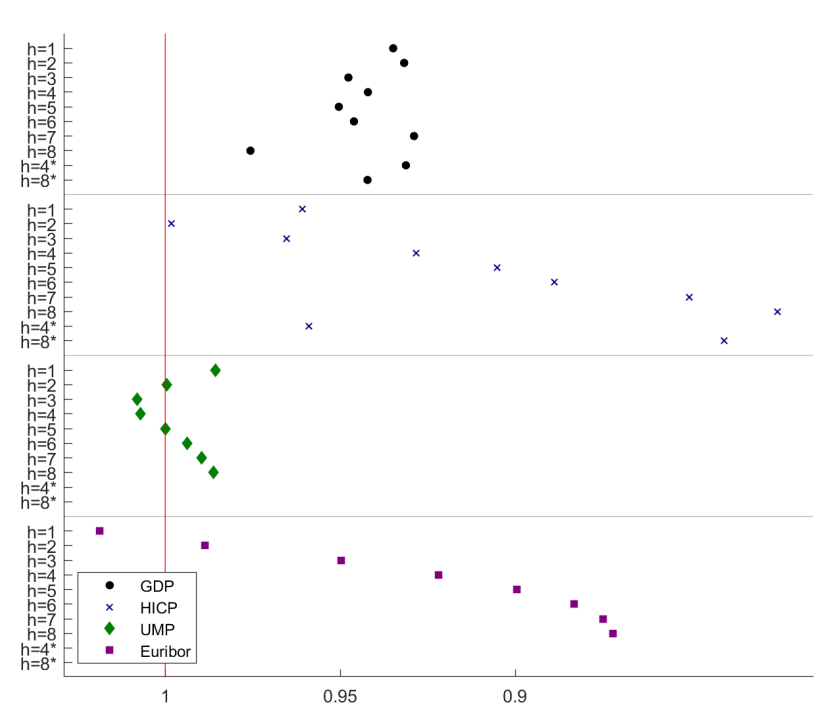

(c) CRPS Ratio

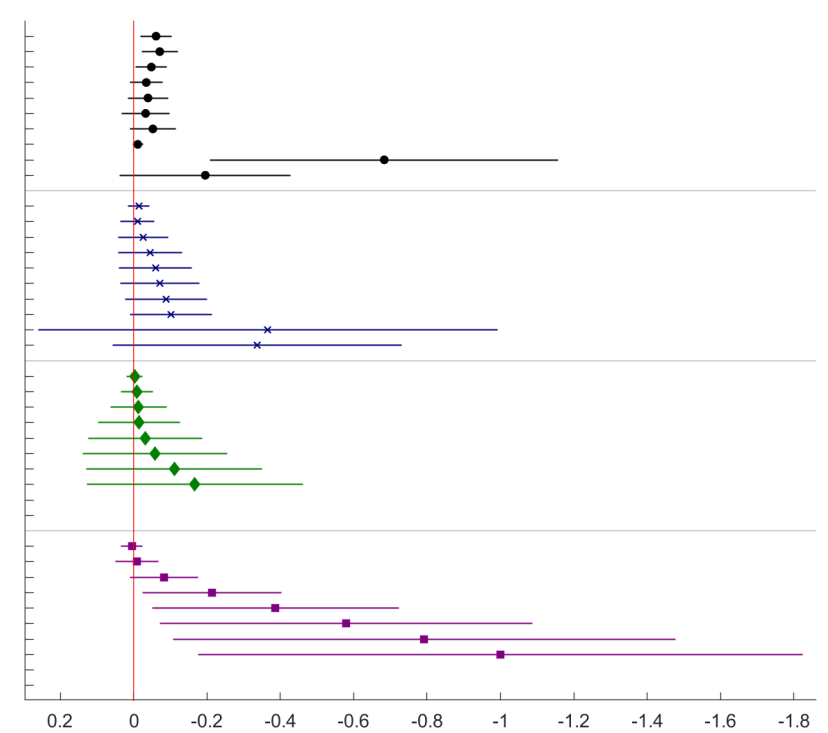

(b) RMSFE Difference

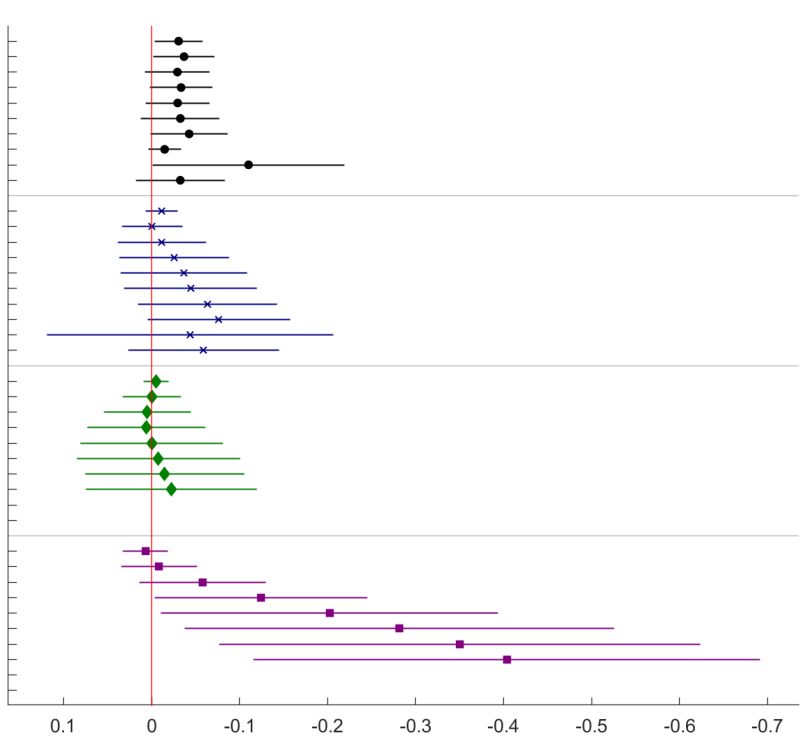

(d) CRPS Difference

Note: The figure shows results when tilting the TVP-VAR predictions jointly towards the one- and two-year-ahead SPF forecasts of real GDP growth, HICP inflation and the unemployment rate. Panel (a) and Panel (c) display the RMSFE ratio of the tilted TVP-VAR and the raw TVP-VAR. A number smaller than one implies that the tilted TVP-VAR outperforms the raw BVAR. Panel (b) and Panel (d) display the RMSFE loss differential of the tilted BVAR minus the raw TVP-VAR. A number smaller than zero implies that the tilted TVP-VAR outperforms the raw TVP-VAR. The axis label $h$ denotes the forecast horizon. The horizontal lines centered around a marker denote $90 \%$ confidence intervals, for which the variance is estimated using Bartlett kernel weights with bandwidth equal to the forecast horizon $h$. To improve legibility, all RSMFE loss differentials of the Euribor have been normalized by the respective value for $h=8 *$.

The stronger forecasting improvement of tilting when looking at density forecasts, instead of point forecasts, is mainly driven by a sharper tilted predictive density. The histograms in Figure 6 display the predictive densities of the TVP-VAR and of the tilted TVP-VAR, when tilting towards all three variables jointly, for the 2013:Q3 SPF round. The tilted and soft conditioned distributions are less dispersed and centered around the realizations. 
Figure 6: Tilted and non-tilted TVP-VAR predictive densities for the 2013:Q3 SPF round

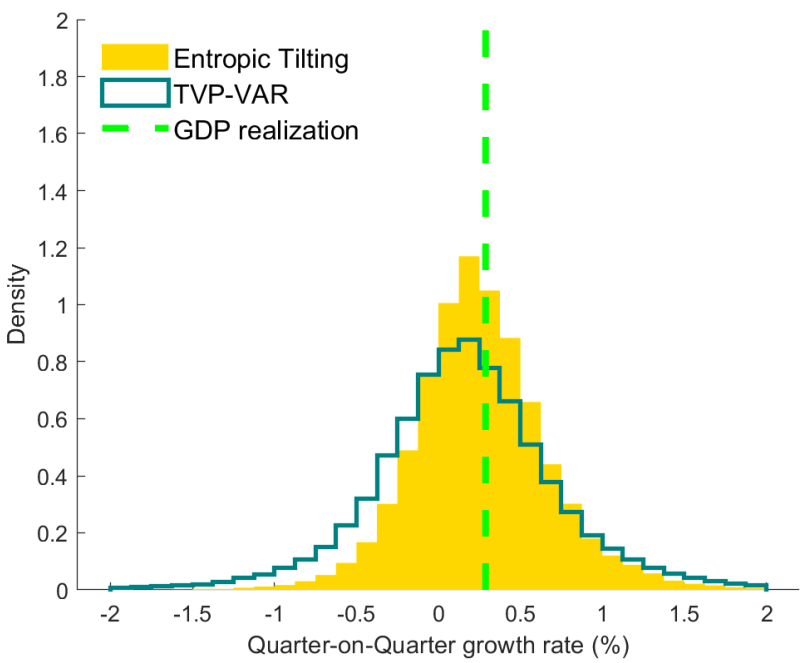

(a) Entropic tilting

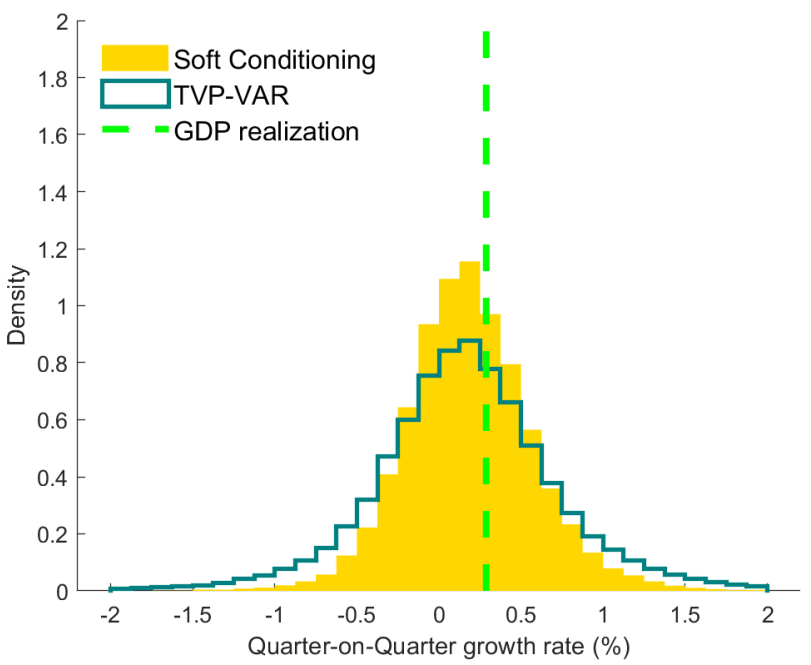

(b) Soft conditioning

Note: Panel (a) displays the histograms of the predictive distribution of GDP growth on 2013:Q4 over 2013:Q3 produced by the TVP-VAR and the tilted TVP-VAR (when tilting towards all three SPF forecasts jointly), for the 2013:Q3 SPF round. The TVP-VAR model's latest observation corresponds to 2013:Q2, and its forecast horizon is $h=2$ quarters. Panel (b) shows analogous results for soft conditioning. The vertical dashed lines indicate the realizations.

\subsection{BVAR with Larger Information Set}

Bańbura et al. (2015) showed that large BVARs are a viable tool for predictions when a large set of predictors is available. We produced real-time forecasts using a medium-sized BVAR with 14 variables. To additionally allow for time-variation in the volatility we used the model proposed by Chan (2018), which contains a common stochastic volatility component. ${ }^{20}$ Following Chan (2018), the own-lag coefficients of variables that are stationary are shrunk towards zero, whereas the coefficients of the first own-lag of variables in levels are shrunk torwards one. ${ }^{21}$ We adopt the lag specification of $p=4$, used by Bańbura et al. (2015) and Chan (2018). Table 8 gives a description of the variables used and which transformation we applied before estimation of the model.

Figure 7 shows the results for the case of jointly tilting real GDP growth, HICP inflation and the unemployment rate. Tables C.5 and C.6 and give detailed results for all four cases of tilting and Appendix $C$ displays results for soft conditioning. We observe that using the information of the SPF survey improves the point and density forecasts of a BVAR, even when the BVAR uses a large set of predictors. Improvements are large for GDP growth in the short horizon and large for HICP inflation, the unemployment rate and the Euribor for longer horizons. Overall, the results are similar to the baseline four-variable BVAR case.

\footnotetext{
${ }^{20}$ The priors that we use are taken from Chan (2018), to which we refer for further details. The priors on the autoregressive coefficients are very similar to Bańbura et al. (2015), but the overall model differs as Bańbura et al. (2015) do not have a common stochastic volatility component.

${ }^{21}$ Results are robust to shrinking level variables towards 0.8 instead of one, as in the baseline specification of the four-variable BVAR.
} 
Figure 7: Results when tilting the large BVAR towards the one- and-two-year-ahead SPF forecast

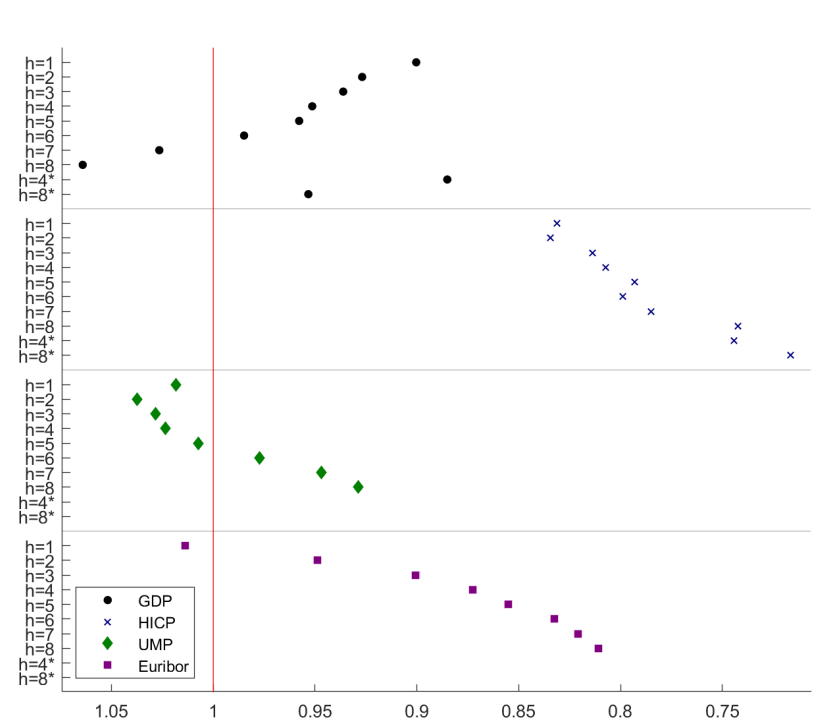

(a) RMSFE Ratio

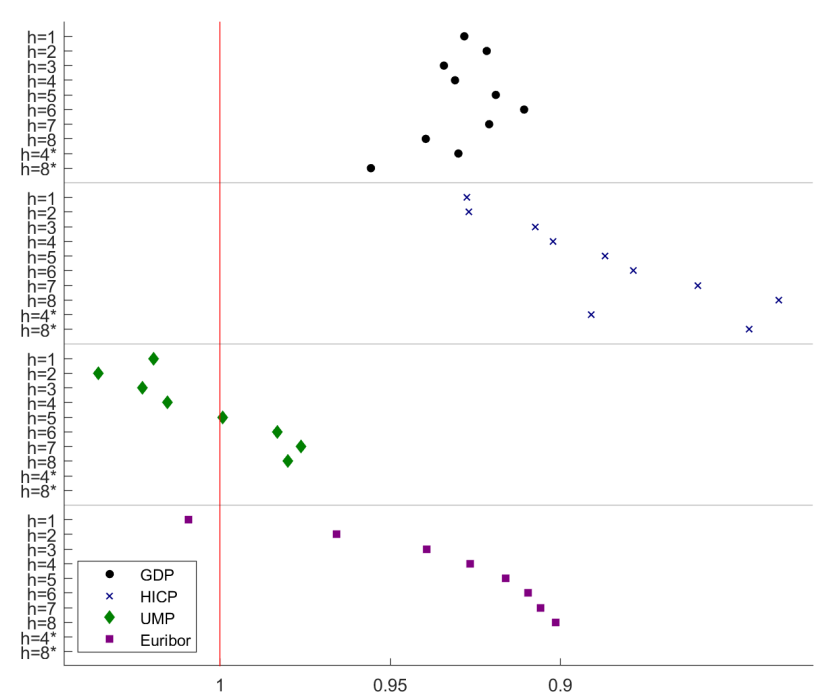

(c) CRPS Ratio

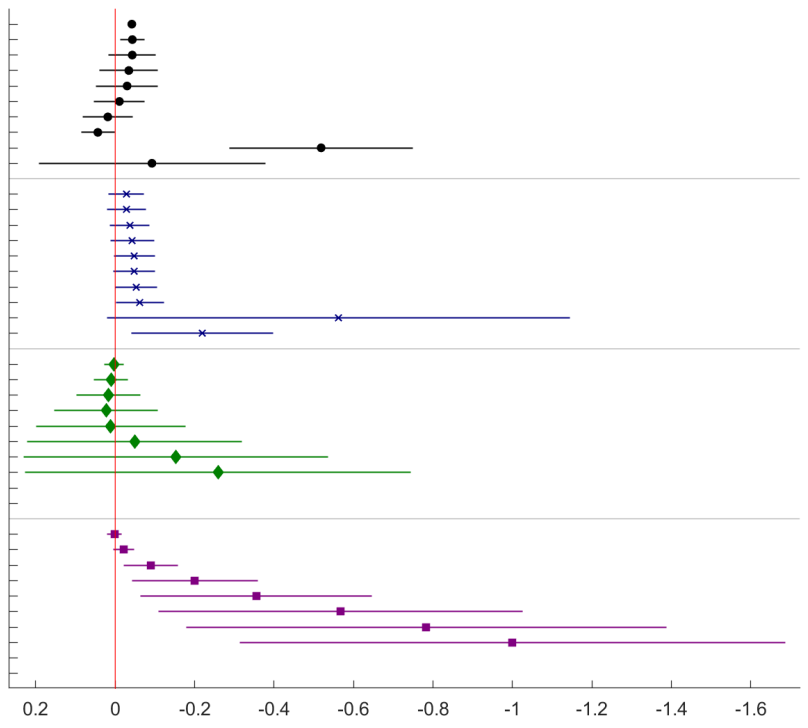

(b) RMSFE Difference

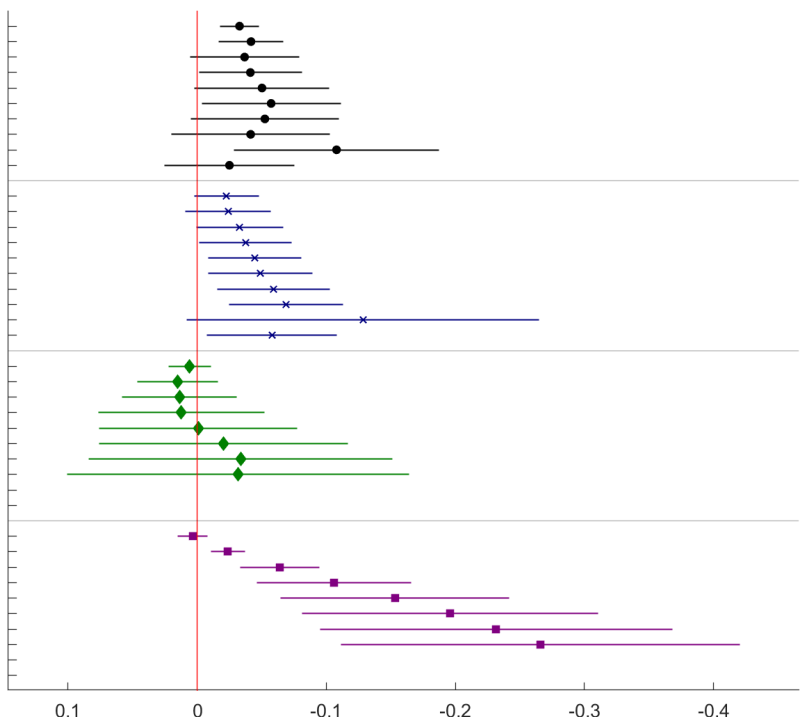

(d) CRPS Difference

Note: The points display the average of the RMSE loss differential of the tilted large BVAR minus the raw large BVAR for different horizons. A negative number implies that the tilted large BVAR outperforms the raw BVAR. The horizontal lines centered around a marker denote $90 \%$ confidence intervals, for which the variance is estimated using Bartlett kernel weights with bandwidth equal to the forecast horizon $h$. Panel (a) displays the results when tilting GDP, Panel (b) when tilting HICP, Panel (c) when tilting the Unemployment rate and Panel (d) when tilting all three variables jointly. To improve legibility, all RSMFE loss differentials of the Euribor have been normalized by the respective value for $h=8^{*}$. 
Table 8: Dataset for BVAR with 14 variables

\begin{tabular}{lccc}
\hline Variable & Data source & Transformation & Identifier \\
\hline Real GDP & ECB RTD & $\Delta$ log-level & RTD.Q.S0.S.G_GDPM_TO_C.E \\
Real Personal Consumption & ECB RTD & $\Delta \log$-level & RTD.Q.S0.S.G_FCHI_TO_C.E \\
Final Government Consumption & ECB RTD & $\Delta$ log-level & RTD.Q.S0.S.G_FCGG_TO_C.E \\
Real Exports & ECB RTD & $\Delta \log$-level & RTD.Q.S0.S.G_XGS_TO_C.E \\
Real Imports & ECB RTD & $\Delta \log$-level & RTD.Q.S0.S.G_MGS_TO_C.E \\
Real Gross Capital Formation & ECB RTD & $\Delta \log$-level & RTD.Q.S0.S.G_GFCF_TO_C.E \\
HICP & ECB RTD & $\Delta \log$-level & RTD.M.S0.N.P_C_OV.X \\
Unemployment Rate & ECB RTD & level & RTD.M.S0.S.L_UNETO.F \\
3-month Euribor & AWM & level & STN \\
10-year benchmark yield & AWM & level & LTN \\
US-EUR Exchange Rate & AWM & level & EXR \\
Commodity Price Index excl. Oil & AWM & $\log$-level & PCOMU \\
Oil Price & AWM & $\log$-level & POILU \\
Euro Stoxx 50 & ECB RTD & $\Delta \log$-level & RTD.M.S0.N.C_DJE50.X \\
\hline
\end{tabular}

Note: ECB RTD denotes the ECB Real Time database and AWM denotes the Area Wide Model database. The column Transformation indicates which variable entered the model in levels, log-levels or in log-differences.

\section{Conclusion}

In a real-time, out-of-sample forecasting exercise based on euro area macroeconomic aggregates, we incorporated survey expectations into the predictive density of a BVAR with stochastic volatility. The forecasting gains are economically and statistically significant when tilting real GDP growth, HICP inflation and the unemployment rate, both individually and jointly. The gains are mainly, but not exclusively driven by using the information of the point predictions. In addition, the incorporation of survey information for one variable exhibits spillover effects to other variables. Overall, the results are in line with previous findings regarding the predictive accuracy of surveys of professional forecasters as well as the usefulness of entropic tilting and soft conditioning. However, different from the existing literature, we impose the restrictions on quarterly year-on-year growth rates, and thereby restrict the possible paths over several horizons.

We documented a bias in real GDP growth forecasts of the BVAR for the period after (but not before) the Great Recession, which we interpreted as a structural change. In turn, the SPF panelists were better than the benchmark macroeconometric model to adapt to the slow GDP growth recovery period after the crisis, which provides evidence that survey expectations can considerably improve model-based forecasts in times of distress and structural change. We consider the tilting procedure as a simple way to correct possible misspecifications of an otherwise useful benchmark model. The results are robust to several alternative specifications, including a time-varying parameter VAR with stochastic volatility, a larger BVAR, estimations in levels and using the hierarchical model of Giannone et al. (2015) with dummy observation priors. 


\section{References}

Altavilla, C., Giacomini, R., and Ragusa, G. (2017). Anchoring the yield curve using survey expectations. Journal of Applied Econometrics, 32(6):1055-1068.

Andersson, M., Palmqvist, S., and Waggoner, D. (2010). Density-conditional forecasts in dynamic multivariate models. Working Paper 247, Sveriges Riksbank.

Ang, A., Bekaert, G., and Wei, M. (2007). Do macro variables, asset markets, or surveys forecast inflation better? Journal of Monetary Economics, 54(4):1163-1212.

Angelini, E., Lalik, M., Lenza, M., and Paredes, J. (2019). Mind the gap: a multi-country BVAR benchmark for the Eurosystem projections. Working Paper 2227, ECB.

Antolín-Díaz, J., Petrella, I., and Rubio-Ramírez, J. (2019). Structural Scenario Analysis with SVARSs. Working Paper.

Bańbura, M., Giannone, D., and Lenza, M. (2015). Conditional forecasts and scenario analysis with vector autoregressions for large cross-sections. International Journal of Forecasting, 31(3):739-756.

Bańbura, M., Giannone, D., and Reichlin, L. (2010). Large Bayesian vector auto regressions. Journal of Applied Econometrics, 25(1):71-92.

Bańbura, M. and van Vlodrop, A. (2018). Forecasting with Bayesian Vector Autoregressions with Time Variation in the Mean. Technical Report 18-025/IV, Tinbergen Institute.

Carriero, A., Clark, T. E., and Marcellino, M. (2015). Bayesian VARs: Specification Choices and Forecast Accuracy. Journal of Applied Econometrics, 30(1):46-73.

Chan, J. C. C. (2018). Large Bayesian VARs: A Flexible Kronecker Error Covariance Structure. Journal of Business \& Economic Statistics, pages 1-12.

Clark, T. E. and Ravazzolo, F. (2015). Macroeconomic Forecasting Performance under Alternative Specifications of Time-Varying Volatility. Journal of Applied Econometrics, 30(4):551-575.

D'Agostino, A., Gambetti, L., and Giannone, D. (2013). Macroeconomic forecasting and structural change. Journal of Applied Econometrics, 28(1):82-101.

Del Negro, M. and Primiceri, G. E. (2015). Time Varying Structural Vector Autoregressions and Monetary Policy: A Corrigendum. The Review of Economic Studies, 82(4):1342-1345.

Del Negro, M. and Schorfheide, F. (2013). DSGE Model-Based Forecasting. In Elliott, G. and Timmermann, A., editors, Handbook of Economic Forecasting, volume 2-A, pages 57 - 140. Elsevier, Amsterdam.

Diebold, F. X. and Mariano, R. S. (1995). Comparing Predictive Accuracy. Journal of Business E Economic Statistics, 13(3):253.

Dieppe, A., Legrand, R., and van Roye, B. (2016). The BEAR Toolbox. Working Paper 1934, European Central Bank. 
European Central Bank (2019). Results of the third special questionnaire for participants in the ECB Survey of Professional Forecasters. Report, European Central Bank.

Fagan, G., Henry, J., and Mestre, R. (2001). An Area-wide Model (AWM) for the euro area. Working Paper 42, European Central Bank.

Faust, J. and Wright, J. H. (2009). Comparing Greenbook and Reduced Form Forecasts Using a Large Realtime Dataset. Journal of Business E Economic Statistics, 27(4):468-479.

Genre, V., Kenny, G., Meyler, A., and Timmermann, A. (2013). Combining expert forecasts: Can anything beat the simple average? International Journal of Forecasting, 29(1):108-121.

Giacomini, R. and Ragusa, G. (2014). Theory-coherent forecasting. Journal of Econometrics, 182(1):145-155.

Giannone, D., Henry, J., Lalik, M., and Modugno, M. (2012). An Area-Wide Real-Time Database for the Euro Area. The Review of Economics and Statistics, 94(4):1000-1013.

Giannone, D., Lenza, M., and Primiceri, G. E. (2015). Prior Selection for Vector Autoregressions. Review of Economics and Statistics, 97(2):436-451.

Giannone, D., Lenza, M., and Primiceri, G. E. (2019). Priors for the Long Run. Journal of the American Statistical Association, 114(526):565-580.

Giordani, P. and Söderlind, P. (2003). Inflation forecast uncertainty. European Economic Review, 47(6):1037-1059.

Gneiting, T., Balabdaoui, F., and Raftery, A. E. (2007). Probabilistic forecasts, calibration and sharpness. Journal of the Royal Statistical Society: Series B (Statistical Methodology), 69(2):243-268.

Hersbach, H. (2000). Decomposition of the Continuous Ranked Probability Score for Ensemble Prediction Systems. Weather and Forecasting, 15(5):559-570.

Jarociński, M. and Lenza, M. (2018). An Inflation-Predicting Measure of the Output Gap in the Euro Area. Journal of Money, Credit and Banking, 50(6):1189-1224.

Kenny, G., Kostka, T., and Masera, F. (2014). How Informative are the Subjective Density Forecasts of Macroeconomists? Journal of Forecasting, 33(3):163-185.

Kenny, G., Kostka, T., and Masera, F. (2015). Can Macroeconomists Forecast Risk? Event-Based Evidence from the Euro-Area SPF. International Journal of Central Banking, 11(4):1-46.

Koop, G. and Potter, S. M. (2011). Time varying VARs with inequality restrictions. Journal of Economic Dynamics and Control, 35(7):1126-1138.

Krüger, F., Clark, T. E., and Ravazzolo, F. (2017). Using Entropic Tilting to Combine BVAR Forecasts With External Nowcasts. Journal of Business \& Economic Statistics, 35(3):470-485.

Lengwiler, Y. (2017). X-13 Toolbox for Matlab, Version 1.33.

McAdam, P. and Warne, A. (2019). Euro area real-time density forecasting with financial or labor market frictions. International Journal of Forecasting, 35(2):580-600. 
Newey, W. K. and West, K. D. (1987). A Simple, Positive Semi-Definite, Heteroskedasticity and Autocorrelation Consistent Covariance Matrix. Econometrica, 55(3):703-708.

Primiceri, G. E. (2005). Time Varying Structural Vector Autoregressions and Monetary Policy. The Review of Economic Studies, 72(3):821-852.

Quaedvlieg, R. (2019). Multi-Horizon Forecast Comparison. Journal of Business E Economic Statistics, 0(0):1-14.

Robertson, J. C., Tallman, E. W., and Whiteman, C. H. (2005). Forecasting Using Relative Entropy. Journal of Money, Credit and Banking, 37(3):20.

Schwarz, G. (1978). Estimating the Dimension of a Model. The Annals of Statistics, 6(2):461-464.

Stark, T. (2010). Realistic Evaluation of Real-Time Forecasts in the Survey of Professional Forecasters. Research Rap, Special Report, Federal Reserve Bank of Philadelphia.

Tallman, E. W. and Zaman, S. (2018). Combining Survey Long-Run Forecasts and Nowcasts with BVAR Forecasts Using Relative Entropy. Working Paper 1809, Federal Reserve Bank of Cleveland.

U.S. Census Bureau (2017). X-13ARIMA-SEATS Seasonal Adjustment Program. U.S. Census Bureau.

Waggoner, D. F. and Zha, T. (1999). Conditional Forecasts in Dynamic Multivariate Models. The Review of Economics and Statistics, 81(4):639-651. 


\section{Appendix A Multi-Horizon Forecast Results}

The results displayed Tables 1, 3, C.3 and C.5 are based on horizon-by-horizon tests and are consequently subject to a multiple testing problem. Therefore, Tables A.1 and A.2 show results of the multi-horizon forecast comparison test of Quaedvlieg (2019), which tests for superior predictive ability of the quarter-on-quarter forecasts while controlling the family-wise error rate of the multiple hypothesis tests. We conduct the test for horizon 1 to 4 and 1 to 8 (note that this is different from the year-on-year tests of $h=4^{*}$ and $h=8^{*}$. Tables A.1 and A.2 show results for tilting different variables. Positive (bold) numbers indicate superior predictive ability of the tilted BVAR relative to the raw BVAR and ${ }^{*}, * *, * * *$ denote statistical significance resulting from the bootstrap procedure of Quaedvlieg (2019). The multi-horizon test confirms the findings in Tables 1, 3, C.3 and C.5. We interpret these results with some caution as the assumptions for the asymptotic theory of the multi-horizon forecast comparison test are a rolling window estimation scheme, i.e. the preservation of parameter estimation error, whereas our BVAR is based on a recursive window estimation scheme because of the small initial sample.

Table A.1: Entropic tilting versus BVAR: Multi-Horizon Test

\begin{tabular}{|c|c|c|c|c|c|c|c|c|}
\hline & \multicolumn{8}{|c|}{ Panel A. Tilting GDP } \\
\hline & \multicolumn{4}{|c|}{ RMSFE } & \multicolumn{4}{|c|}{ CRPS } \\
\hline & GDP & HICP & UMP & INTR & GDP & HICP & UMP & INTR \\
\hline$h=1-$ & $1.89^{* * *}$ & -0.65 & $1.73^{* *}$ & $1.44^{* *}$ & $2.22^{* * *}$ & -0.89 & $1.50^{* *}$ & $1.37^{* *}$ \\
\hline$h=1-8$ & 1.00 & -0.67 & $1.37^{*}$ & 0.71 & $1.51^{*}$ & -0.70 & 1.29 & $1.19^{*}$ \\
\hline
\end{tabular}

\begin{tabular}{|c|c|c|c|c|c|c|c|c|}
\hline & \multirow{2}{*}{\multicolumn{4}{|c|}{ RMSFE }} & \multirow{2}{*}{\multicolumn{4}{|c|}{ CRPS }} \\
\hline & & & & & & & & \\
\hline & GDP & HICP & UMP & INTR & GDP & HICP & UMP & INTR \\
\hline & $1.02^{* *}$ & $0.81^{*}$ & $1.15^{* *}$ & $1.56^{* *}$ & $1.19^{* *}$ & $0.92^{*}$ & $0.98^{*}$ & $1.26^{* *}$ \\
\hline & 0.98 & 1.15 & 0.31 & $2.03^{* *}$ & 1.33 & 1.25 & 0.09 & $1.88^{* *}$ \\
\hline
\end{tabular}

\begin{tabular}{|c|c|c|c|c|c|c|c|c|}
\hline & \multicolumn{8}{|c|}{ Panel C. Tilting Unemployment } \\
\hline & & $R M$ & SFE & & & CRI & & \\
\hline & GDP & HICP & UMP & INTR & GDP & HICP & UMP & INTR \\
\hline & 0.49 & -2.00 & $1.84^{* * *}$ & 0.22 & -0.24 & -1.68 & $2.63^{* * *}$ & 0.05 \\
\hline & -0.78 & -1.06 & $2.41^{* *}$ & 0.27 & -1.01 & -0.89 & $3.17^{* * *}$ & 0.49 \\
\hline
\end{tabular}

\begin{tabular}{|c|c|c|c|c|c|c|c|c|}
\hline & \multicolumn{8}{|c|}{ Panel D. Tilting Jointly } \\
\hline & & $R M$ & & & & $C R$ & & \\
\hline & GDP & HICP & UMP & INTR & GDP & HICP & UMP & INTR \\
\hline 1 & $1.71^{* *}$ & $0.79 *$ & $1.92^{* * *}$ & $1.65^{* *}$ & $1.95^{* *}$ & $0.81^{*}$ & $2.86^{* * *}$ & $1.54^{* *}$ \\
\hline$h=1-$ & 0.75 & 1.10 & $2.39^{* *}$ & $1.82^{* *}$ & 1.22 & 1.15 & $3.16^{* * *}$ & $1.83^{* *}$ \\
\hline
\end{tabular}

Note: The table shows the results of the "multi-horizon forecast comparison test" by Quaedvlieg (2019). Positive (bold) numbers imply that the tilted BVAR improved over the raw BVAR. Asterisks ${ }^{*},{ }^{* *}$ and ${ }^{* * *}$ imply statistical significance of the one-sided multi-horizon test at the $10 \%, 5 \%, 1 \%$ level. The evaluation sample size is $P=72$ quarters. The rows $\mathrm{h}=1-4$ and $\mathrm{h}=1-8$ show the results when applying the multi-horizon test to forecast horizons $\mathrm{h}=1$ to 4 and $\mathrm{h}=1$ to 8 respectively. 
Table A.2: Soft Conditioning versus BVAR: Multi-Horizon Test

\begin{tabular}{|c|c|c|c|c|c|c|c|c|}
\hline \multirow{5}{*}{$\begin{array}{l}h=1-4 \\
h=1-8\end{array}$} & \multicolumn{8}{|c|}{ Panel A. Tilting GDP } \\
\hline & \multicolumn{4}{|c|}{ RMSFE } & \multicolumn{4}{|c|}{ CRPS } \\
\hline & GDP & HICP & UMP & INTR & GDP & $\mathrm{HICP}$ & UMP & INTR \\
\hline & $2.13^{* * *}$ & -0.41 & $1.78^{* *}$ & $1.61^{* *}$ & $2.34^{* * *}$ & -0.74 & $1.02^{*}$ & $1.97^{* *}$ \\
\hline & 1.27 & -0.50 & $1.44^{*}$ & 1.02 & $1.53^{*}$ & -0.78 & 0.74 & $1.67^{* *}$ \\
\hline & \multicolumn{8}{|c|}{ Panel B. Tilting HICP } \\
\hline & \multicolumn{4}{|c|}{ RMSFE } & \multicolumn{4}{|c|}{ CRPS } \\
\hline & GDP & HICP & UMP & INTR & GDP & $\mathrm{HICP}$ & UMP & INTR \\
\hline $\mathrm{h}=1-4$ & $1.29^{* *}$ & $1.38^{* *}$ & $1.21^{* *}$ & $1.89^{* *}$ & $1.51^{* *}$ & $1.15^{* *}$ & 0.32 & $1.70^{* *}$ \\
\hline \multirow[t]{4}{*}{$h=1-8$} & 1.13 & $1.79^{* *}$ & 0.31 & $2.38^{* *}$ & $1.64^{*}$ & $1.64^{* *}$ & -1.11 & $2.38^{* *}$ \\
\hline & \multicolumn{8}{|c|}{ Panel C. Tilting Unemployment } \\
\hline & \multicolumn{4}{|c|}{ RMSFE } & \multicolumn{4}{|c|}{ CRPS } \\
\hline & GDP & $\mathrm{HICP}$ & UMP & INTR & GDP & $\mathrm{HICP}$ & UMP & INTR \\
\hline$h=1-4$ & $1.82^{* *}$ & -1.05 & $1.75^{* * *}$ & -0.75 & $1.85^{* *}$ & -1.22 & $1.19^{* *}$ & -0.78 \\
\hline \multirow[t]{4}{*}{$h=1-8$} & $1.33^{*}$ & -0.96 & $1.79^{* *}$ & -1.02 & $1.71^{*}$ & -1.16 & 0.85 & -1.08 \\
\hline & \multicolumn{8}{|c|}{ Panel D. Tilting Jointly } \\
\hline & \multicolumn{4}{|c|}{ RMSFE } & \multicolumn{4}{|c|}{ CRPS } \\
\hline & GDP & HICP & UMP & INTR & GDP & HICP & UMP & INTR \\
\hline$h=1-4$ & $2.32^{* * *}$ & $1.20^{* *}$ & $2.07^{* * *}$ & $1.97^{* *}$ & $2.64^{* * *}$ & $0.78^{*}$ & $1.36^{* *}$ & $2.38^{* * *}$ \\
\hline$h=1-8$ & $1.34^{*}$ & $1.60^{* *}$ & $1.82^{* *}$ & $2.29 * * *$ & $1.65^{*}$ & 1.17 & 0.73 & $2.88^{* * *}$ \\
\hline
\end{tabular}

Note: The table shows the results of the "multi-horizon forecast comparison test" by Quaedvlieg (2019). Positive (bold) numbers imply that the tilted BVAR improved over the raw BVAR. Asterisks *, ${ }^{* *}$ and ${ }^{* * *}$ imply statistical significance of the one-sided multihorizon test at the $10 \%, 5 \%, 1 \%$ level. The evaluation sample size is $P=72$ quarters. The rows $h=1-4$ and $h=1-8$ show the results when applying the multi-horizon test to forecast horizons $\mathrm{h}=1$ to 4 and $\mathrm{h}=1$ to 8 respectively. 


\section{Appendix B Tilting Towards One-Year-Ahead SPF Forecasts Only}

The previous results are based on tilting towards the one- and two-year-ahead forecasts of the SPF jointly. In turn, Figure B.1 shows results for tilting all three variables jointly towards the one-year-ahead SPF predictions only. Results for individually tilting GDP, HICP and the unemployment rate are displayed in Table B.1 and Table B.2. The forecasting improvement are strong in the short-run for GDP, extend to longer horizons in the case of tilting the inflation rate, the unemployment rate and the Euribor and are often economically relevant in size and statistically significant. Appendix $C$ shows additional results for soft conditioning, which are again qualitatively similar.

Table B.1: Entropic tilting One-Year-Ahead versus BVAR forecasts: RMSFE

\begin{tabular}{|c|c|c|c|c|c|c|c|c|c|c|}
\hline Forecast horizon & $h=1$ & $h=2$ & $h=3$ & $h=4$ & $h=5$ & $h=6$ & $h=7$ & $h=8$ & $h=4^{*}$ & $h=8^{*}$ \\
\hline & \multicolumn{10}{|c|}{ Panel A. BVAR } \\
\hline GDP & 0.55 & 0.59 & 0.59 & 0.58 & 0.58 & 0.58 & 0.57 & 0.57 & 1.68 & 1.93 \\
\hline HICP & 0.26 & 0.27 & 0.29 & 0.30 & 0.31 & 0.31 & 0.31 & 0.31 & 0.92 & 1.07 \\
\hline Unemployment & 0.29 & 0.46 & 0.65 & 0.83 & 1.00 & 1.16 & 1.30 & 1.43 & $N / A$ & $N / A$ \\
\hline \multirow[t]{2}{*}{ Euribor } & 0.35 & 0.66 & 0.94 & 1.20 & 1.44 & 1.64 & 1.82 & 1.97 & $N / A$ & $N / A$ \\
\hline & \multicolumn{10}{|c|}{ Panel B. Tilting GDP } \\
\hline GDP & $0.80^{* *}$ & $0.86^{*}$ & 0.91 & 0.98 & 1.01 & 1.01 & 1.01 & 1.01 & $0.81^{* *}$ & 1.01 \\
\hline HICP & 1.00 & 1.00 & 1.01 & 1.00 & 1.00 & 1.00 & 1.00 & 1.00 & 1.01 & 1.01 \\
\hline Unemployment & 0.95 & $0.92^{*}$ & $0.91^{*}$ & 0.92 & 0.94 & 0.95 & 0.96 & 0.97 & $N / A$ & $N / A$ \\
\hline \multirow[t]{2}{*}{ Euribor } & 0.99 & 0.97 & 0.97 & 0.97 & $0.97^{*}$ & 0.98 & 0.98 & 0.99 & $N / A$ & $N / A$ \\
\hline & \multicolumn{10}{|c|}{ Panel C. Tilting HICP } \\
\hline GDP & 0.99 & 0.99 & 0.99 & 1.00 & $1.00^{*}$ & 1.00 & 1.00 & 1.00 & 0.99 & 1.00 \\
\hline НICP & 0.94 & 0.98 & 0.93 & 0.93 & 0.93 & 0.92 & $0.92^{* * *}$ & $0.93^{*}$ & 0.91 & $0.90^{*}$ \\
\hline Unemployment & 0.99 & 1.00 & 1.00 & $1.01^{* * *}$ & $1.02^{* * *}$ & $1.02^{* * *}$ & $1.02^{* * *}$ & $1.02^{* * *}$ & $N / A$ & $N / A$ \\
\hline \multirow[t]{2}{*}{ Euribor } & 0.98 & $0.95^{*}$ & $0.93^{*}$ & $0.93^{* *}$ & $0.93^{* *}$ & $0.93^{* *}$ & $0.94^{* *}$ & $0.94^{* *}$ & $N / A$ & $N / A$ \\
\hline & \multicolumn{10}{|c|}{ Panel D. Tilting Unemployment } \\
\hline GDP & 0.96 & 0.97 & 0.99 & 0.98 & 0.99 & $1.03^{* *}$ & 1.04 & $1.03^{*}$ & 0.96 & $0.99^{* *}$ \\
\hline HICP & $1.02^{* *}$ & $1.02^{*}$ & 1.02 & 1.02 & 1.02 & 1.02 & 1.01 & 1.03 & $1.03^{* *}$ & 1.03 \\
\hline Unemployment & 0.93 & 0.88 & $0.86^{* *}$ & $0.86^{* *}$ & $0.87^{* *}$ & $0.88^{* *}$ & $0.89^{* *}$ & $0.91^{* *}$ & $N / A$ & $N / A$ \\
\hline \multirow[t]{2}{*}{ Euribor } & 1.05 & 1.02 & 1.01 & 1.02 & 1.01 & 1.00 & 0.98 & 0.98 & $N / A$ & $N / A$ \\
\hline & \multicolumn{10}{|c|}{ Panel E. Tilting GDP, HICP and Unemployment } \\
\hline GDP & $0.79^{* *}$ & $0.86^{*}$ & 0.92 & 1.00 & 1.01 & 1.01 & $1.03^{*}$ & 1.06 & $0.82^{* *}$ & 1.00 \\
\hline HICP & 0.95 & 0.97 & 0.94 & 0.93 & 0.94 & 0.94 & 0.95 & 0.97 & 0.91 & 0.93 \\
\hline Unemployment & $0.89^{*}$ & $0.84^{*}$ & $0.83^{* *}$ & $0.86^{* *}$ & $0.88^{* *}$ & $0.89^{* *}$ & $0.90^{* *}$ & $0.91^{* *}$ & $N / A$ & $N / A$ \\
\hline Euribor & 0.95 & $0.88^{* *}$ & $0.88^{* *}$ & $0.91^{*}$ & $0.92^{*}$ & $0.93^{*}$ & $0.93^{*}$ & 0.94 & $N / A$ & $N / A$ \\
\hline
\end{tabular}

Note: Panel A displays the raw BVAR RMSFE. Panels B to E show the ratios of the RMSFE of the entropic tilted BVAR to the raw BVAR. Numbers in bold imply that the tilted BVAR improves over the raw BVAR. Asterisks *, ${ }^{* *}$ and ${ }^{* * *}$ imply statistical significance of a two-sided Diebold and Mariano (1995) test of equal predictive ability at the 10\%, 5\%, 1\% level. The variance is estimated using Bartlett kernel weights with bandwidth equal to the forecast horizon $h$. The evaluation sample size is $P=72$ quarters. The columns $h=4^{*}$ and $h=8^{*}$ show the results for the one- and two-year-ahead, year-on-year predictions, respectively. 
Figure B.1: Results when tilting towards the one-year-ahead SPF forecast
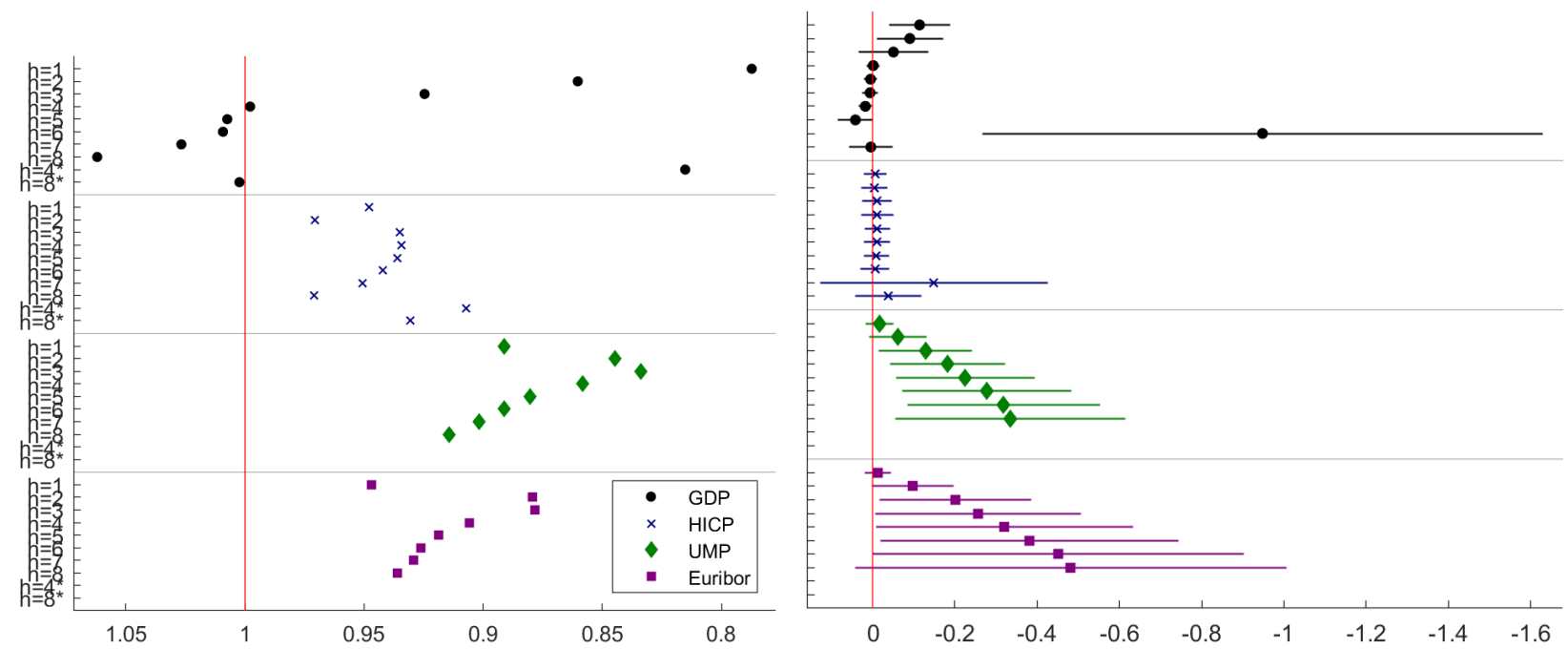

(a) RMSFE Ratio

(b) RMSFE Difference
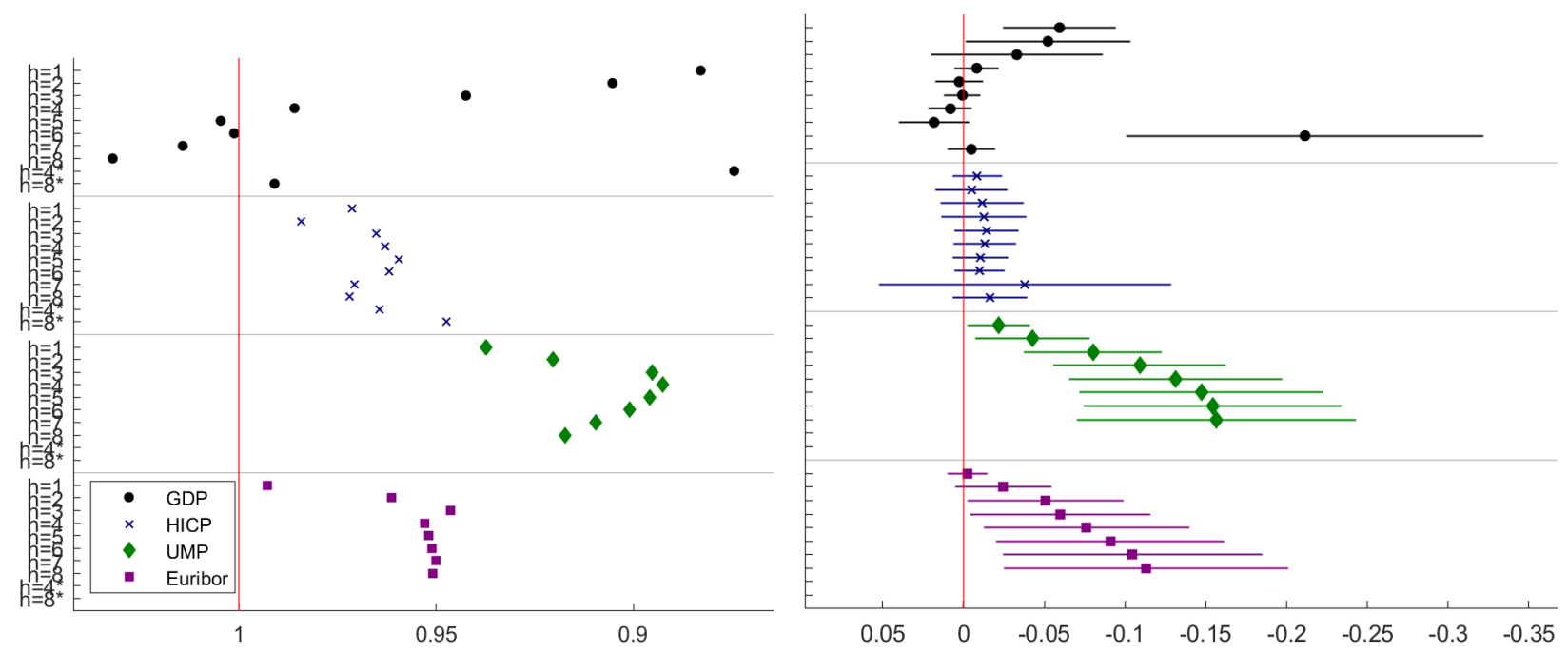

(c) CRPS Ratio

(d) CRPS Difference

Note: The figure shows results when tilting the BVAR predictions jointly towards the one-year-ahead SPF forecasts of real GDP growth, HICP inflation and the unemployment rate. Panel (a) and Panel (c) display the RMSFE ratio of the tilted BVAR and the raw BVAR. A number smaller than one implies that the tilted BVAR outperforms the raw BVAR. Panel (b) and Panel (d) display the RMSFE loss differential of the tilted BVAR minus the raw BVAR. A number smaller than zero implies that the tilted BVAR outperforms the raw BVAR. The axis label $h$ denotes the forecast horizon. The horizontal lines centered around a marker denote $90 \%$ confidence intervals, for which the variance is estimated using Bartlett kernel weights with bandwidth equal to the forecast horizon $h$. To improve legibility, all RSMFE loss differentials of the Euribor have been normalized by the respective value for $h=8 *$. 
Table B.2: Entropic tilting One-Year-Ahead versus BVAR forecasts: CRPS

\begin{tabular}{|c|c|c|c|c|c|c|c|c|c|c|}
\hline \multirow[t]{2}{*}{ Forecast horizon } & $h=1$ & $h=2$ & $h=3$ & $h=4$ & $h=5$ & $h=6$ & $h=7$ & $h=8$ & $h=4^{*}$ & $h=8^{*}$ \\
\hline & \multicolumn{10}{|c|}{ Panel A. BVAR } \\
\hline GDP & 0.27 & 0.29 & 0.29 & 0.29 & 0.29 & 0.29 & 0.28 & 0.28 & 0.90 & 1.08 \\
\hline HICP & 0.15 & 0.16 & 0.17 & 0.17 & 0.18 & 0.18 & 0.18 & 0.18 & 0.54 & 0.63 \\
\hline Unemployment & 0.18 & 0.28 & 0.40 & 0.54 & 0.66 & 0.78 & 0.89 & 0.99 & $N / A$ & $N / A$ \\
\hline \multirow[t]{2}{*}{ Euribor } & 0.17 & 0.32 & 0.49 & 0.65 & 0.81 & 0.95 & 1.07 & 1.18 & $N / A$ & $N / A$ \\
\hline & \multicolumn{10}{|c|}{ Panel B. Tilting GDP } \\
\hline GDP & $0.79^{* * *}$ & $0.81^{*}$ & 0.86 & 0.96 & 0.99 & 0.99 & 1.00 & 1.00 & $0.76^{* * *}$ & 0.99 \\
\hline HICP & 1.00 & 1.01 & 1.02 & 1.01 & 1.00 & 1.00 & 1.00 & 1.00 & 1.01 & 1.00 \\
\hline Unemployment & 0.96 & 0.93 & 0.92 & 0.93 & 0.94 & 0.95 & 0.96 & 0.97 & $N / A$ & $N / A$ \\
\hline \multirow[t]{2}{*}{ Euribor } & 0.99 & 0.98 & 0.97 & $0.96^{*}$ & $0.96^{* *}$ & $0.96^{* *}$ & 0.96 & 0.96 & $N / A$ & $N / A$ \\
\hline & \multicolumn{10}{|c|}{ Panel C. Tilting HICP } \\
\hline GDP & 0.99 & 0.99 & 0.99 & 1.00 & $0.99^{*}$ & $1.00^{*}$ & 1.00 & 1.00 & 0.99 & $0.99^{*}$ \\
\hline $\mathrm{HICP}$ & 0.93 & 0.98 & 0.92 & 0.92 & $0.92^{*}$ & $0.92^{* *}$ & $0.92^{* *}$ & $0.93^{* * *}$ & 0.93 & $0.89^{*}$ \\
\hline Unemployment & 0.99 & 1.00 & $1.01^{*}$ & $1.02^{* *}$ & $1.02^{* *}$ & $1.02^{* *}$ & $1.03^{* *}$ & $1.03^{* *}$ & $N / A$ & $N / A$ \\
\hline \multirow[t]{2}{*}{ Euribor } & 0.99 & 0.96 & 0.95 & 0.94 & 0.94 & $0.94^{*}$ & $0.94^{*}$ & $0.94^{*}$ & $N / A$ & $N / A$ \\
\hline & \multicolumn{10}{|c|}{ Panel D. Tilting Unemployment } \\
\hline GDP & 0.97 & 1.00 & 1.00 & 1.00 & 1.02 & 1.01 & $1.07^{*}$ & $1.06^{* *}$ & $0.95^{* *}$ & 0.99 \\
\hline HICP & $1.02^{*}$ & $1.02^{*}$ & 1.01 & 1.02 & 1.02 & 1.02 & 1.00 & 1.01 & $1.04^{* *}$ & 1.01 \\
\hline Unemployment & $0.91^{*}$ & $0.89^{*}$ & $0.84^{* * *}$ & $0.82^{* * *}$ & $0.82^{* * *}$ & $0.82^{* * *}$ & $0.83^{* * *}$ & $0.85^{* * *}$ & $N / A$ & $N / A$ \\
\hline \multirow[t]{2}{*}{ Euribor } & 1.07 & 1.03 & 1.01 & 1.01 & 0.98 & 0.96 & 0.96 & 0.96 & $N / A$ & $N / A$ \\
\hline & \multicolumn{10}{|c|}{ Panel E. Tilting GDP, HICP and Unemployment } \\
\hline GDP & $0.78^{* * *}$ & $0.82^{*}$ & 0.89 & 0.97 & 1.01 & 1.00 & 1.03 & $1.06^{*}$ & $0.76^{* * *}$ & 0.98 \\
\hline $\mathrm{HICP}$ & 0.94 & 0.97 & 0.93 & 0.93 & 0.92 & 0.93 & 0.94 & 0.94 & 0.93 & 0.90 \\
\hline Unemployment & $0.88^{* *}$ & $0.85^{* *}$ & $0.80^{* * *}$ & $0.80^{* * *}$ & $0.80^{* * *}$ & $0.81^{* * *}$ & $0.83^{* * *}$ & $0.84^{* * *}$ & $N / A$ & $N / A$ \\
\hline Euribor & 0.99 & 0.92 & $0.90^{*}$ & $0.91^{*}$ & $0.91^{* *}$ & $0.90^{* *}$ & $0.90^{* *}$ & $0.90^{* *}$ & $N / A$ & $N / A$ \\
\hline
\end{tabular}

Note: Panel A displays the raw BVAR CRPS. Panels B to E show the ratios of the CRPS of the entropic tilted BVAR to the raw BVAR. Numbers in bold imply that the tilted BVAR improves over the raw BVAR. Asterisks ${ }^{*},{ }^{* *}$ and ${ }^{* * *}$ imply statistical significance of a two-sided Diebold and Mariano (1995) test of equal predictive ability at the $10 \%, 5 \%, 1 \%$ level. The variance is estimated using Bartlett kernel weights with bandwidth equal to the forecast horizon $h$. The evaluation sample size is $P=72$ quarters. The columns $h=4^{*}$ and $h=8^{*}$ show the results for the one- and two-year-ahead, year-on-year predictions, respectively. 
Table B.3: Soft Conditioning versus BVAR forecasts: RMSFE

\begin{tabular}{|c|c|c|c|c|c|c|c|c|c|c|}
\hline \multirow[t]{2}{*}{ Forecast horizon } & $h=1$ & $h=2$ & $h=3$ & $h=4$ & $h=5$ & $h=6$ & $h=7$ & $h=8$ & $h=4 *$ & $h=8 *$ \\
\hline & \multicolumn{10}{|c|}{ Panel A. BVAR } \\
\hline GDP & 0.55 & 0.59 & 0.59 & 0.58 & 0.58 & 0.58 & 0.57 & 0.57 & 1.68 & 1.93 \\
\hline HICP & 0.26 & 0.27 & 0.29 & 0.30 & 0.31 & 0.31 & 0.31 & 0.31 & 0.92 & 1.07 \\
\hline Unemployment & 0.29 & 0.46 & 0.65 & 0.83 & 1.00 & 1.16 & 1.30 & 1.43 & $N / A$ & $N / A$ \\
\hline \multirow[t]{2}{*}{ Euribor } & 0.35 & 0.66 & 0.94 & 1.20 & 1.44 & 1.64 & 1.82 & 1.97 & $N / A$ & $N / A$ \\
\hline & \multicolumn{10}{|c|}{ Panel B. Soft Conditioning GDP } \\
\hline GDP & $0.87^{* *}$ & $0.91^{* * *}$ & 0.93 & 0.99 & 1.00 & 1.01 & 1.01 & 1.01 & $0.88^{* *}$ & 1.00 \\
\hline HICP & 1.00 & 1.00 & 1.00 & 1.00 & 1.00 & 1.00 & 1.00 & 1.00 & 1.00 & 1.00 \\
\hline Unemployment & 0.97 & $0.95^{*}$ & $0.95^{* *}$ & $0.96^{*}$ & 0.97 & 0.97 & 0.98 & 0.98 & $N / A$ & $N / A$ \\
\hline \multirow[t]{2}{*}{ Euribor } & 0.99 & 0.98 & 0.98 & $0.98^{*}$ & $0.98^{* *}$ & $0.99^{* *}$ & 0.99 & 0.99 & $N / A$ & $N / A$ \\
\hline & \multicolumn{10}{|c|}{ Panel C. Soft Conditioning HICP } \\
\hline GDP & 1.00 & 1.00 & 1.00 & 1.00 & $1.00^{* *}$ & 1.00 & 1.00 & 1.00 & $1.00^{*}$ & 1.00 \\
\hline HICP & $0.97^{*}$ & 0.98 & 0.96 & 0.95 & $0.96^{*}$ & $0.96^{*}$ & $0.96^{*}$ & 0.96 & 0.94 & $0.94^{*}$ \\
\hline Unemployment & 1.00 & 1.00 & 1.00 & $1.00^{* *}$ & $1.00^{* *}$ & $1.01^{* *}$ & $1.01^{* * *}$ & $1.01^{* * *}$ & $N / A$ & $N / A$ \\
\hline \multirow[t]{2}{*}{ Euribor } & 0.99 & $0.98^{*}$ & $0.97^{*}$ & $0.97^{*}$ & $0.97^{* *}$ & $0.97^{* *}$ & $0.97^{* *}$ & $0.98^{* *}$ & $N / A$ & $N / A$ \\
\hline & \multicolumn{10}{|c|}{ Panel D. Soft Conditioning Unemployment } \\
\hline GDP & $0.99^{*}$ & $0.99^{*}$ & 1.00 & 1.00 & 1.00 & 1.00 & 1.00 & 1.01 & 1.00 & 1.00 \\
\hline HICP & 1.00 & 1.00 & 1.01 & 1.01 & 1.01 & 1.01 & 1.01 & 1.01 & 1.01 & 1.02 \\
\hline Unemployment & 0.98 & 0.97 & 0.97 & 0.97 & 0.97 & 0.98 & 0.98 & 0.98 & $N / A$ & $N / A$ \\
\hline \multirow[t]{2}{*}{ Euribor } & 1.01 & 1.01 & 1.01 & 1.01 & 1.01 & 1.01 & 1.01 & 1.00 & $N / A$ & $N / A$ \\
\hline & \multicolumn{10}{|c|}{ Panel E. Soft Conditioning GDP, HICP and Unemployment } \\
\hline GDP & $0.87^{* *}$ & $0.90^{* *}$ & 0.93 & 0.99 & 1.00 & 1.01 & 1.01 & 1.01 & $0.88^{* *}$ & 1.00 \\
\hline HICP & 0.97 & 0.98 & 0.96 & 0.96 & 0.97 & 0.97 & 0.97 & 0.97 & 0.95 & 0.96 \\
\hline Unemployment & $0.96^{*}$ & $0.94^{* *}$ & $0.93^{*}$ & $0.94^{*}$ & 0.96 & 0.97 & 0.98 & 0.98 & $N / A$ & $N / A$ \\
\hline Euribor & 0.99 & $0.96^{*}$ & $0.95^{* *}$ & $0.95^{* * *}$ & $0.96^{* * *}$ & $0.96^{* * *}$ & $0.97^{* * *}$ & $0.97^{* * *}$ & $N / A$ & $N / A$ \\
\hline
\end{tabular}

Note: Panel A displays the raw BVAR RMSFE. Panels B to E show the ratios of the RMSFE of the soft conditioned BVAR to the raw BVAR. Numbers in bold imply that the soft conditioned BVAR improves over the raw BVAR. Asterisks *, ** and ${ }^{* * *}$ imply statistical significance of a two-sided Diebold and Mariano (1995) test of equal predictive ability at the $10 \%, 5 \%, 1 \%$ level. The variance is estimated using Bartlett kernel weights with bandwidth equal to the forecast horizon $h$. The evaluation sample size is $P=72$ quarters. The columns $h=4^{*}$ and $h=8^{*}$ show the results for the one- and two-year-ahead, year-on-year predictions, respectively. 
Table B.4: Soft Conditioning versus BVAR forecasts: CRPS

\begin{tabular}{|c|c|c|c|c|c|c|c|c|c|c|}
\hline \multirow[t]{3}{*}{ Forecast horizon } & $h=1$ & $h=2$ & $h=3$ & $h=4$ & $h=5$ & $h=6$ & $h=7$ & $h=8$ & $h=4 *$ & $h=8 *$ \\
\hline & \multicolumn{10}{|c|}{ Panel A. BVAR } \\
\hline & 0.27 & 0.29 & 0.29 & 0.29 & 0.29 & 0.29 & 0.28 & 0.28 & 0.90 & 1.08 \\
\hline HICP & 0.15 & 0.16 & 0.17 & 0.17 & 0.18 & 0.18 & 0.18 & 0.18 & 0.54 & 0.63 \\
\hline Unemployment & 0.18 & 0.28 & 0.40 & 0.54 & 0.66 & 0.78 & 0.89 & 0.99 & $N / A$ & $N / A$ \\
\hline \multirow[t]{2}{*}{ Euribor } & 0.17 & 0.32 & 0.49 & 0.65 & 0.81 & 0.95 & 1.07 & 1.18 & $N / A$ & $N / A$ \\
\hline & \multicolumn{10}{|c|}{ Panel B. Soft Conditioning GDP } \\
\hline GDP & $0.87^{* * *}$ & $0.88^{* *}$ & $0.91^{*}$ & 0.98 & 0.99 & 1.00 & 1.00 & 1.00 & $0.89^{* *}$ & 1.00 \\
\hline HICP & 1.00 & 1.00 & 1.01 & 1.00 & 1.00 & 1.00 & 1.00 & 1.00 & 1.01 & 1.00 \\
\hline Unemployment & 0.98 & 0.97 & 0.96 & 0.97 & 0.97 & 0.98 & 0.98 & 0.99 & $N / A$ & $N / A$ \\
\hline \multirow[t]{2}{*}{ Euribor } & 0.99 & 0.98 & $0.97^{*}$ & $0.97^{* *}$ & $0.97^{* *}$ & $0.98^{*}$ & 0.98 & 0.98 & $N / A$ & $N / A$ \\
\hline & \multicolumn{10}{|c|}{ Panel C. Soft Conditioning HICP } \\
\hline GDP & 1.00 & 0.99 & 0.99 & $1.00^{* *}$ & $1.00^{* *}$ & $1.00^{* * *}$ & $1.00^{*}$ & $1.00^{*}$ & 0.99 & 1.00 \\
\hline HICP & 0.97 & 0.99 & $0.96^{*}$ & $0.96^{*}$ & 0.96 & $0.96^{*}$ & $0.96^{*}$ & $0.97^{*}$ & 0.97 & 0.95 \\
\hline Unemployment & 1.00 & 1.00 & $1.00^{* * *}$ & $1.01^{* * *}$ & $1.01^{* * *}$ & $1.01^{* * *}$ & $1.01^{* * *}$ & $1.01^{* * *}$ & $N / A$ & $N / A$ \\
\hline \multirow[t]{2}{*}{ Euribor } & 1.00 & 0.98 & 0.97 & $0.97^{*}$ & $0.97^{* *}$ & $0.97^{* *}$ & $0.97^{* *}$ & $0.98^{* *}$ & $N / A$ & $N / A$ \\
\hline & \multicolumn{10}{|c|}{ Panel D. Soft Conditioning Unemployment } \\
\hline GDP & $0.99^{*}$ & 0.99 & 1.00 & 1.00 & 1.00 & 1.00 & 1.00 & 1.01 & 1.00 & 1.00 \\
\hline HICP & 1.00 & 1.00 & 1.01 & 1.01 & 1.01 & 1.01 & 1.01 & 1.01 & $1.01^{*}$ & 1.02 \\
\hline Unemployment & 0.99 & 0.98 & 0.97 & 0.98 & 0.98 & 0.98 & 0.98 & 0.99 & $N / A$ & $N / A$ \\
\hline \multirow[t]{2}{*}{ Euribor } & 1.00 & 1.00 & 1.00 & 1.00 & 1.00 & 1.00 & 1.00 & 1.00 & $N / A$ & $N / A$ \\
\hline & \multicolumn{10}{|c|}{ Panel E. Soft Conditioning GDP, HICP and Unemployment } \\
\hline GDP & $0.87^{* * *}$ & $0.87^{* * *}$ & $0.91^{*}$ & $0.98^{*}$ & 0.99 & 1.00 & 1.00 & 1.00 & $0.89^{* *}$ & 1.00 \\
\hline HICP & 0.98 & 0.99 & 0.97 & 0.96 & 0.97 & 0.97 & 0.97 & 0.97 & 0.99 & 0.97 \\
\hline Unemployment & 0.97 & 0.96 & 0.95 & 0.96 & 0.97 & 0.98 & 0.99 & 0.99 & $N / A$ & $N / A$ \\
\hline Euribor & 0.99 & $0.97^{* *}$ & $0.95^{* * *}$ & $0.95^{* * *}$ & $0.95^{* * *}$ & $0.95^{* * *}$ & $0.96^{* * *}$ & $0.96^{* *}$ & $N / A$ & $N / A$ \\
\hline
\end{tabular}

Note: Panel A displays the raw BVAR CRPS. Panels B to E show the ratios of the CRPS of the soft conditioned BVAR to the raw BVAR. Numbers in bold imply that the soft conditioned BVAR improves over the raw BVAR. Asterisks ${ }^{*}, * *$ and ${ }^{* * *}$ imply statistical significance of a two-sided Diebold and Mariano (1995) test of equal predictive ability at the $10 \%, 5 \%, 1 \%$ level. The variance is estimated using Bartlett kernel weights with bandwidth equal to the forecast horizon $h$. The evaluation sample size is $P=72$ quarters. The columns $h=4^{*}$ and $h=8^{*}$ show the results for the one- and two-year-ahead, year-on-year predictions, respectively.

\section{Appendix C Alternative Model Specifications}

\section{C.1 BVAR without GDP jumping-off}

To ensure that our results are not driven by the properties of the ECB nowcasts, we also produce forecasts of the BVAR without a jumping-off procedure. To accommodate the timing of the GDP releases (see the Data section of the main paper for details), the timing of the BVAR predictions has to change. As before, for the first forecast, the in-sample estimation of the BVAR is based on the 1999:Q1 vintage (before the release of 1998:Q4 real GDP data), which is aligned with the SPF 1999:Q1 predictions, and the in-sample estimation ranges from 1991:Q1 to 1998:Q3. Different from 
the jumping-off approach, the first prediction of the BVAR, denoted by $h=0$, is for 1998:Q4 and based on GDP, HICP, unemployment and Euribor realizations of 1998:Q3. We then substitute the released values of HICP, the unemployment rate and the Euribor for their forecasts for 1998:Q4 and retain the GDP forecast for which there is no realized data available. In other words, we use a jumping-off approach for all variables but GDP growth. An alternative would be to use a Kalman filter to impute the missing values of real GDP growth at the end of the sample (see Bańbura et al. (2015) for an example). While using the Kalman filter would be the most efficient approach as it uses the information of the most recent HICP and unemployment releases to impute the missing GDP value, we would expect that the differences to the results presented here are small.

As before, the horizon $h=1$ corresponds to a nowcast, that is predictions for the quarter of the survey round. The horizon $h=0$ corresponds to a backcast, but only for GDP which we subsequently don't evaluate. The total out-of-sample forecast errors we evaluate range from 1999:Q1 to 2016:Q4 for the $h=1$ predictions, and from 2000:Q1 to 2018:Q3 for $h=8$ predictions, i.e. the total out-of-sample size is again $P=72$ for all horizons.

Table C.1 summarizes and illustrates the timing of the data available to SPF panelists and the BVAR model, along with the forecast targets through the example of the 2007:Q4 survey round.

Table C.1: Timing of the ECB-SPF and the BVAR model, without GDP jumping-off, for the 2007:Q4 round

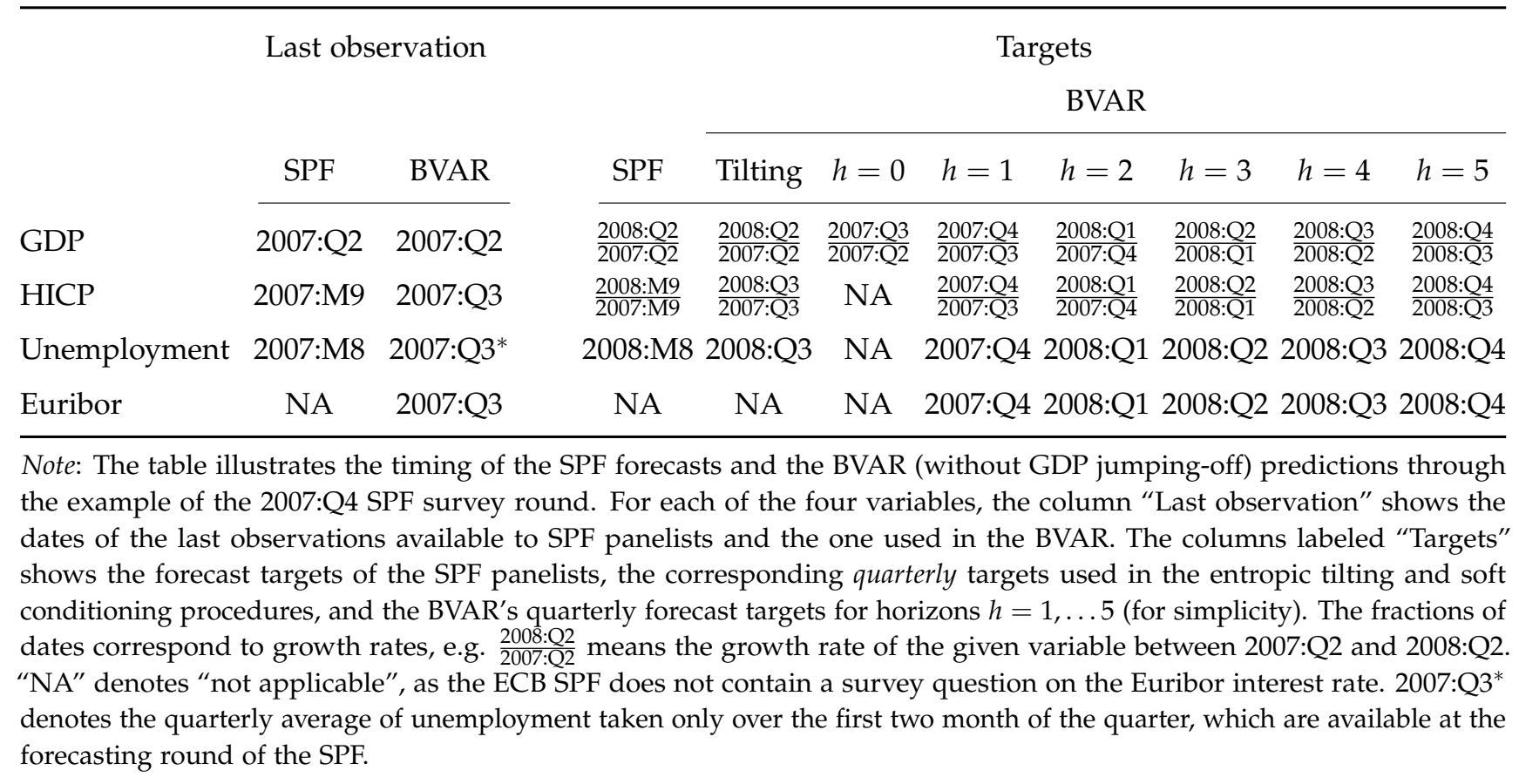


Table C.2: Entropic tilting versus BVAR without jumping-off forecasts: RMSFE

\begin{tabular}{|c|c|c|c|c|c|c|c|c|c|c|}
\hline \multirow[t]{3}{*}{ Forecast horizon } & $h=1$ & $h=2$ & $h=3$ & $h=4$ & $h=5$ & $h=6$ & $h=7$ & $h=8$ & $h=4 *$ & $h=8 *$ \\
\hline & \multicolumn{10}{|c|}{ Panel A. BVAR } \\
\hline & 0.57 & 0.59 & 0.59 & 0.58 & 0.58 & 0.58 & 0.57 & 0.57 & 1.93 & 1.92 \\
\hline HICP & 0.26 & 0.27 & 0.29 & 0.30 & 0.31 & 0.31 & 0.31 & 0.31 & 0.92 & 1.07 \\
\hline Unemployment & 0.29 & 0.47 & 0.65 & 0.84 & 1.01 & 1.16 & 1.31 & 1.43 & $N / A$ & $N / A$ \\
\hline \multirow[t]{2}{*}{ Euribor } & 0.36 & 0.68 & 0.96 & 1.22 & 1.46 & 1.66 & 1.84 & 1.99 & $N / A$ & $N / A$ \\
\hline & \multicolumn{10}{|c|}{ Panel B. Tilting GDP } \\
\hline GDP & $0.75^{* *}$ & $0.85^{* *}$ & 0.89 & 0.97 & 1.00 & 1.01 & 1.01 & 1.02 & $0.71^{* *}$ & 1.00 \\
\hline $\mathrm{HICP}$ & 1.00 & 1.00 & 1.01 & 1.01 & 1.01 & 1.01 & 1.01 & 1.01 & 1.01 & 1.02 \\
\hline Unemployment & $0.95^{*}$ & $0.91^{*}$ & $0.90^{* *}$ & $0.91^{*}$ & 0.92 & 0.93 & 0.95 & 0.96 & $N / A$ & $N / A$ \\
\hline \multirow[t]{2}{*}{ Euribor } & $0.94^{* *}$ & $0.91^{* * *}$ & $0.89^{* * *}$ & $0.91^{* * *}$ & $0.93^{* *}$ & $0.95^{*}$ & 0.97 & 0.97 & $N / A$ & $N / A$ \\
\hline & \multicolumn{10}{|c|}{ Panel C. Tilting HICP } \\
\hline GDP & 0.98 & 0.98 & 0.98 & 0.99 & 0.99 & 1.00 & 1.00 & 1.00 & 0.98 & 0.99 \\
\hline HICP & 0.93 & 0.98 & 0.93 & 0.93 & 0.92 & 0.92 & 0.91 & 0.91 & 0.91 & 0.88 \\
\hline Unemployment & 0.98 & 0.97 & 0.98 & 0.98 & 0.99 & 0.99 & 1.00 & 1.01 & $N / A$ & $N / A$ \\
\hline \multirow[t]{2}{*}{ Euribor } & 0.96 & 0.92 & $0.88^{*}$ & $0.86^{*}$ & $0.85^{*}$ & $0.84^{* *}$ & $0.84^{* *}$ & $0.85^{* *}$ & $N / A$ & $N / A$ \\
\hline & \multicolumn{10}{|c|}{ Panel D. Tilting Unemployment } \\
\hline GDP & 0.97 & 0.97 & 0.99 & 0.98 & $1.02^{* * *}$ & $1.09^{*}$ & $1.06^{*}$ & $1.04^{* *}$ & $0.93^{* *}$ & 1.00 \\
\hline HICP & $1.04^{* *}$ & $1.02^{* *}$ & 1.02 & 1.02 & 1.02 & 1.02 & 1.02 & 1.03 & $1.03^{* *}$ & 1.04 \\
\hline Unemployment & 0.92 & 0.88 & $0.85^{* *}$ & $0.85^{* *}$ & $0.86^{* * *}$ & $0.87^{* * *}$ & $0.88^{* * *}$ & $0.89^{* * *}$ & $N / A$ & $N / A$ \\
\hline \multirow[t]{2}{*}{ Euribor } & 1.02 & $0.95^{*}$ & 0.95 & 0.98 & 0.98 & 0.97 & 0.97 & 0.98 & $N / A$ & $N / A$ \\
\hline & \multicolumn{10}{|c|}{ Panel E. Tilting GDP, HICP and Unemployment } \\
\hline GDP & $0.77^{* *}$ & $0.86^{*}$ & 0.91 & 1.01 & 1.00 & 1.01 & 1.01 & $1.06^{* * *}$ & $0.71^{* *}$ & 1.00 \\
\hline HICP & 0.94 & 0.96 & 0.94 & 0.95 & 0.91 & 0.92 & 0.92 & 0.93 & 0.91 & 0.88 \\
\hline Unemployment & $0.91^{*}$ & $0.85^{*}$ & $0.84^{* *}$ & $0.85^{* *}$ & $0.87^{* * *}$ & $0.87^{* * *}$ & $0.89^{* * *}$ & $0.90^{* * *}$ & $N / A$ & $N / A$ \\
\hline Euribor & $0.90^{*}$ & $0.82^{* *}$ & $0.80^{* *}$ & $0.82^{* *}$ & $0.83^{* *}$ & $0.85^{* *}$ & $0.85^{* *}$ & $0.86^{* *}$ & $N / A$ & $N / A$ \\
\hline
\end{tabular}

Note: Panel A displays the raw BVAR without jumping-off RMSFE. Panels B to E show the ratios of the RMSFE of the entropic tilted BVAR without jumping-off to the raw BVAR without jumping-off. Numbers in bold imply that the tilted BVAR without jumping-off improves over the raw BVAR without jumping-off. Asterisks ${ }^{*},{ }^{* *}$ and ${ }^{* *}$ imply statistical significance of a two-sided Diebold and Mariano (1995) test of equal predictive ability at the $10 \%, 5 \%, 1 \%$ level. The variance is estimated using Bartlett kernel weights with bandwidth equal to the forecast horizon $h$. The evaluation sample size is $P=72$ quarters. The columns $h=4^{*}$ and $h=8^{*}$ show the results for the one- and two-year-ahead, year-on-year predictions, respectively. 
Table C.3: Entropic tilting versus BVAR without jumping-off forecasts: CRPS

\begin{tabular}{|c|c|c|c|c|c|c|c|c|c|c|}
\hline \multirow[t]{2}{*}{ Forecast horizon } & $h=1$ & $h=2$ & $h=3$ & $h=4$ & $h=5$ & $h=6$ & $h=7$ & $h=8$ & $h=4 *$ & $h=8 *$ \\
\hline & \multicolumn{10}{|c|}{ Panel A. BVAR } \\
\hline GDP & 0.28 & 0.29 & 0.29 & 0.29 & 0.29 & 0.29 & 0.28 & 0.28 & 1.06 & 1.08 \\
\hline HICP & 0.15 & 0.16 & 0.17 & 0.17 & 0.18 & 0.18 & 0.18 & 0.18 & 0.54 & 0.63 \\
\hline Unemployment & 0.18 & 0.28 & 0.41 & 0.54 & 0.67 & 0.78 & 0.89 & 0.99 & $N / A$ & $N / A$ \\
\hline \multirow[t]{2}{*}{ Euribor } & 0.17 & 0.34 & 0.50 & 0.67 & 0.83 & 0.97 & 1.09 & 1.19 & $N / A$ & $N / A$ \\
\hline & \multicolumn{10}{|c|}{ Panel B. Tilting GDP } \\
\hline GDP & $0.74^{* * *}$ & $0.79^{* *}$ & 0.84 & 0.93 & 0.95 & 0.96 & 0.97 & 1.00 & $0.66^{* * *}$ & 0.97 \\
\hline HICP & 1.00 & 1.01 & 1.02 & 1.02 & 1.02 & 1.01 & 1.01 & 1.00 & 1.02 & 1.02 \\
\hline Unemployment & $0.95^{* *}$ & 0.93 & 0.91 & 0.91 & 0.92 & 0.93 & 0.94 & 0.95 & $N / A$ & $N / A$ \\
\hline \multirow[t]{2}{*}{ Euribor } & $0.94^{* *}$ & $0.91^{* * *}$ & $0.89^{* * *}$ & $0.90^{* * *}$ & $0.91^{* * *}$ & $0.93^{* * *}$ & $0.94^{*}$ & 0.94 & $N / A$ & $N / A$ \\
\hline & \multicolumn{10}{|c|}{ Panel C. Tilting HICP } \\
\hline GDP & 0.98 & 0.98 & 0.98 & 0.99 & 0.99 & 0.99 & 0.99 & 1.00 & 0.98 & 0.99 \\
\hline $\mathrm{HICP}$ & $0.93^{*}$ & 0.97 & 0.92 & 0.92 & 0.92 & 0.91 & 0.90 & 0.90 & 0.93 & 0.88 \\
\hline Unemployment & 0.98 & 0.98 & 0.98 & 0.99 & 0.99 & 1.00 & 1.01 & 1.01 & $N / A$ & $N / A$ \\
\hline \multirow[t]{2}{*}{ Euribor } & 0.98 & 0.95 & 0.91 & 0.89 & 0.87 & $0.86^{*}$ & $0.86^{*}$ & $0.86^{*}$ & $N / A$ & $N / A$ \\
\hline & \multicolumn{10}{|c|}{ Panel D. Tilting Unemployment } \\
\hline GDP & 0.99 & 1.01 & 1.01 & 0.99 & $1.04^{* *}$ & $1.06^{* *}$ & $1.09^{*}$ & $1.06^{* *}$ & $0.91^{* * *}$ & 0.99 \\
\hline $\mathrm{HICP}$ & 1.04 & $1.02^{* *}$ & 1.01 & 1.02 & 1.01 & 1.02 & 1.01 & 1.01 & $1.04^{*}$ & 1.01 \\
\hline Unemployment & $0.91^{* *}$ & $0.87^{* *}$ & $0.83^{* * *}$ & $0.81^{* * *}$ & $0.80^{* * *}$ & $0.80^{* * *}$ & $0.81^{* * *}$ & $0.82^{* * *}$ & $N / A$ & $N / A$ \\
\hline \multirow[t]{2}{*}{ Euribor } & 1.05 & 0.97 & 0.96 & 0.97 & 0.96 & 0.95 & 0.95 & 0.96 & $N / A$ & $N / A$ \\
\hline & \multicolumn{10}{|c|}{ Panel E. Tilting GDP, HICP and Unemployment } \\
\hline GDP & $0.76^{* * *}$ & $0.82^{*}$ & 0.88 & 0.96 & 0.96 & 0.97 & 1.00 & 1.04 & $0.67^{* *}$ & 0.97 \\
\hline HICP & 0.94 & 0.95 & 0.94 & 0.96 & 0.92 & 0.91 & 0.91 & 0.92 & 0.93 & 0.89 \\
\hline Unemployment & $0.88^{* * *}$ & $0.84^{* *}$ & $0.79^{* * *}$ & $0.78^{* * *}$ & $0.78^{* * *}$ & $0.79^{* * *}$ & $0.80^{* * *}$ & $0.82^{* * *}$ & $N / A$ & $N / A$ \\
\hline Euribor & 0.94 & $0.86^{*}$ & $0.82^{* *}$ & $0.83^{* *}$ & $0.83^{* *}$ & $0.84^{* *}$ & $0.83^{* *}$ & $0.84^{* *}$ & $N / A$ & $N / A$ \\
\hline
\end{tabular}

Note: Panel A displays the raw BVAR without jumping-off CRPS. Panels B to E show the ratios of the CRPS of the entropic tilted BVAR without jumping-off to the raw BVAR without jumping-off. Numbers in bold imply that the tilted BVAR without jumping-off improves over the raw BVAR without jumping-off. Asterisks ${ }^{*},{ }^{* *}$ and ${ }^{* * *}$ imply statistical significance of a two-sided Diebold and Mariano (1995) test of equal predictive ability at the $10 \%, 5 \%, 1 \%$ level. The variance is estimated using Bartlett kernel weights with bandwidth equal to the forecast horizon $h$. The evaluation sample size is $P=72$ quarters. The columns $h=4^{*}$ and $h=8^{*}$ show the results for the one- and two-year-ahead, year-on-year predictions, respectively. 
Table C.4: Soft Conditioning versus BVAR without jumping-off forecasts: RMSFE

\begin{tabular}{|c|c|c|c|c|c|c|c|c|c|c|}
\hline Forecast horizon & $h=1$ & $h=2$ & $h=3$ & $h=4$ & $h=5$ & $h=6$ & $h=7$ & $h=8$ & $h=4 *$ & $h=8 *$ \\
\hline & \multicolumn{10}{|c|}{ Panel A. BVAR } \\
\hline GDP & 0.57 & 0.59 & 0.59 & 0.58 & 0.58 & 0.58 & 0.57 & 0.57 & 1.93 & 1.92 \\
\hline HICP & 0.26 & 0.27 & 0.29 & 0.30 & 0.31 & 0.31 & 0.31 & 0.31 & 0.92 & 1.07 \\
\hline Unemployment & 0.29 & 0.47 & 0.65 & 0.84 & 1.01 & 1.16 & 1.31 & 1.43 & $N / A$ & $N / A$ \\
\hline \multirow[t]{2}{*}{ Euribor } & 0.36 & 0.68 & 0.96 & 1.22 & 1.46 & 1.66 & 1.84 & 1.99 & $N / A$ & $N / A$ \\
\hline & \multicolumn{10}{|c|}{ Panel B. Soft Conditioning GDP } \\
\hline GDP & $0.82^{* *}$ & $0.88^{* * *}$ & 0.91 & 0.97 & 0.99 & 0.99 & 1.00 & 1.01 & $0.79^{* *}$ & 0.98 \\
\hline НICP & 1.00 & 1.00 & 1.01 & 1.00 & 1.01 & 1.01 & 1.00 & 1.01 & 1.00 & 1.01 \\
\hline Unemployment & $0.96^{*}$ & $0.94^{*}$ & $0.93^{* *}$ & $0.94^{*}$ & 0.95 & 0.96 & 0.96 & 0.97 & $N / A$ & $N / A$ \\
\hline \multirow[t]{2}{*}{ Euribor } & $0.96^{* *}$ & $0.94^{* *}$ & $0.93^{* * *}$ & $0.94^{* * *}$ & $0.95^{* * *}$ & $0.96^{* * *}$ & 0.97 & 0.98 & $N / A$ & $N / A$ \\
\hline & \multicolumn{10}{|c|}{ Panel C. Soft Conditioning HICP } \\
\hline GDP & 0.99 & 0.99 & 0.99 & 0.99 & 1.00 & 1.00 & 1.00 & $1.00^{* *}$ & 0.99 & 1.00 \\
\hline НICP & $0.96^{*}$ & 0.98 & 0.95 & 0.94 & 0.93 & 0.93 & $0.92^{*}$ & $0.93^{*}$ & 0.94 & $0.90^{*}$ \\
\hline Unemployment & 0.99 & 0.99 & 0.99 & 0.99 & 0.99 & 1.00 & 1.00 & 1.00 & $N / A$ & $N / A$ \\
\hline \multirow[t]{2}{*}{ Euribor } & 0.98 & $0.96^{*}$ & $0.94^{*}$ & $0.93^{*}$ & $0.92^{*}$ & $0.92^{* *}$ & $0.92^{* *}$ & $0.92^{* *}$ & $N / A$ & $N / A$ \\
\hline & \multicolumn{10}{|c|}{ Panel D. Soft Conditioning Unemployment } \\
\hline GDP & $0.99^{*}$ & $0.99^{*}$ & 1.00 & 1.00 & 1.00 & 1.00 & 1.00 & 1.01 & $0.99^{* *}$ & 1.00 \\
\hline HICP & 1.00 & 1.00 & 1.00 & 1.00 & 1.01 & 1.01 & 1.01 & 1.01 & 1.00 & 1.02 \\
\hline Unemployment & 0.98 & 0.97 & $0.96^{*}$ & $0.96^{*}$ & $0.97^{*}$ & $0.97^{*}$ & $0.97^{*}$ & $0.97^{*}$ & $N / A$ & $N / A$ \\
\hline \multirow[t]{2}{*}{ Euribor } & 1.01 & 1.00 & 1.00 & 1.01 & 1.01 & 1.01 & 1.01 & $1.01^{*}$ & $N / A$ & $N / A$ \\
\hline & \multicolumn{10}{|c|}{ Panel E. Soft Conditioning GDP, HICP and Unemployment } \\
\hline GDP & $0.81^{* *}$ & $0.88^{* *}$ & 0.91 & 0.97 & 0.99 & 0.99 & 1.00 & 1.02 & $0.79^{* *}$ & 0.98 \\
\hline HICP & 0.97 & 0.98 & 0.96 & 0.95 & 0.94 & 0.93 & $0.93^{*}$ & $0.94^{*}$ & 0.94 & $0.91^{*}$ \\
\hline Unemployment & $0.95^{*}$ & $0.92^{* *}$ & $0.91^{* *}$ & $0.92^{* *}$ & $0.93^{*}$ & 0.94 & 0.95 & 0.96 & $N / A$ & $N / A$ \\
\hline Euribor & $0.95^{*}$ & $0.91^{* *}$ & $0.88^{* * *}$ & $0.88^{* * *}$ & $0.89^{* * *}$ & $0.89^{* * *}$ & $0.91^{* * *}$ & $0.92^{* * *}$ & $N / A$ & $N / A$ \\
\hline
\end{tabular}

Note: Panel A displays the raw BVAR without jumping-off RMSFE. Panels B to E show the ratios of the RMSFE of the soft conditioned BVAR without jumping-off to the raw BVAR without jumping-off. Numbers in bold imply that the soft conditioned BVAR without jumping-off improves over the raw BVAR without jumping-off. Asterisks ${ }^{*},{ }^{* *}$ and ${ }^{* * *}$ imply statistical significance of a two-sided Diebold and Mariano (1995) test of equal predictive ability at the 10\%, 5\%, 1\% level. The variance is estimated using Bartlett kernel weights with bandwidth equal to the forecast horizon $h$. The evaluation sample size is $P=72$ quarters. The columns $h=4^{*}$ and $h=8^{*}$ show the results for the one- and two-year-ahead, year-on-year predictions, respectively. 
Table C.5: Soft Conditioning versus BVAR without jumping-off forecasts: CRPS

\begin{tabular}{|c|c|c|c|c|c|c|c|c|c|c|}
\hline \multirow[t]{2}{*}{ Forecast horizon } & $h=1$ & $h=2$ & $h=3$ & $h=4$ & $h=5$ & $h=6$ & $h=7$ & $h=8$ & $h=4 *$ & $h=8 *$ \\
\hline & \multicolumn{10}{|c|}{ Panel A. BVAR } \\
\hline GDP & 0.28 & 0.29 & 0.29 & 0.29 & 0.29 & 0.29 & 0.28 & 0.28 & 1.06 & 1.08 \\
\hline HICP & 0.15 & 0.16 & 0.17 & 0.17 & 0.18 & 0.18 & 0.18 & 0.18 & 0.54 & 0.63 \\
\hline Unemployment & 0.18 & 0.28 & 0.41 & 0.54 & 0.67 & 0.78 & 0.89 & 0.99 & $N / A$ & $N / A$ \\
\hline \multirow[t]{2}{*}{ Euribor } & 0.17 & 0.34 & 0.50 & 0.67 & 0.83 & 0.97 & 1.09 & 1.19 & $N / A$ & $N / A$ \\
\hline & \multicolumn{10}{|c|}{ Panel B. Soft Conditioning GDP } \\
\hline GDP & $0.81^{* * *}$ & $0.84^{* * *}$ & $0.88^{*}$ & 0.94 & 0.96 & 0.97 & 0.97 & 1.00 & $0.79^{* *}$ & 1.00 \\
\hline HICP & 1.00 & 1.01 & 1.01 & 1.01 & 1.01 & 1.01 & 1.01 & 1.01 & 1.02 & 1.02 \\
\hline Unemployment & 0.98 & 0.96 & 0.95 & 0.95 & 0.96 & 0.97 & 0.97 & 0.98 & $N / A$ & $N / A$ \\
\hline \multirow[t]{2}{*}{ Euribor } & $0.95^{* * *}$ & $0.93^{* * *}$ & $0.92^{* * *}$ & $0.93^{* * *}$ & $0.94^{* * *}$ & $0.95^{* * *}$ & 0.96 & 0.96 & $N / A$ & $N / A$ \\
\hline & \multicolumn{10}{|c|}{ Panel C. Soft Conditioning HICP } \\
\hline GDP & 0.99 & 0.99 & 0.99 & 0.99 & $0.99^{*}$ & $0.99^{* *}$ & $1.00^{* *}$ & 1.00 & 0.99 & 1.00 \\
\hline HICP & 0.97 & 0.98 & $0.96^{*}$ & 0.95 & $0.94^{*}$ & $0.94^{*}$ & $0.93^{*}$ & $0.93^{*}$ & 0.97 & 0.93 \\
\hline Unemployment & 1.00 & 0.99 & 1.00 & 1.00 & 1.00 & 1.01 & $1.01^{*}$ & $1.02^{* *}$ & $N / A$ & $N / A$ \\
\hline \multirow[t]{2}{*}{ Euribor } & 0.99 & 0.97 & 0.95 & 0.94 & $0.93^{*}$ & $0.93^{*}$ & $0.93^{* *}$ & $0.93^{* *}$ & $N / A$ & $N / A$ \\
\hline & \multicolumn{10}{|c|}{ Panel D. Soft Conditioning Unemployment } \\
\hline GDP & $0.99^{*}$ & $0.99^{* *}$ & 1.00 & 1.00 & $1.00^{*}$ & 1.00 & 1.00 & 1.00 & 0.99 & 1.00 \\
\hline HICP & 1.00 & $1.00^{*}$ & 1.00 & 1.01 & 1.01 & 1.01 & 1.01 & 1.01 & $1.01^{*}$ & 1.02 \\
\hline Unemployment & 0.99 & 0.97 & 0.97 & 0.97 & 0.98 & 0.98 & 0.99 & 0.99 & $N / A$ & $N / A$ \\
\hline \multirow[t]{2}{*}{ Euribor } & 1.00 & 1.00 & 1.00 & 1.01 & 1.01 & 1.01 & 1.01 & 1.01 & $N / A$ & $N / A$ \\
\hline & \multicolumn{10}{|c|}{ Panel E. Soft Conditioning GDP, HICP and Unemployment } \\
\hline GDP & $0.80^{* * *}$ & $0.84^{* * *}$ & $0.88^{*}$ & 0.94 & 0.96 & 0.97 & 0.97 & 1.00 & $0.80^{* *}$ & 1.01 \\
\hline HICP & 0.98 & 0.99 & 0.97 & 0.97 & 0.96 & 0.95 & 0.95 & 0.95 & 0.99 & 0.96 \\
\hline Unemployment & $0.97^{*}$ & 0.94 & 0.93 & 0.94 & 0.95 & 0.97 & 0.98 & 0.99 & $N / A$ & $N / A$ \\
\hline Euribor & $0.95^{* *}$ & $0.91^{* * *}$ & $0.89^{* * *}$ & $0.88^{* * *}$ & $0.89^{* * *}$ & $0.89^{* * *}$ & $0.90^{* * *}$ & $0.91^{* *}$ & $N / A$ & $N / A$ \\
\hline
\end{tabular}

Note: Panel A displays the raw BVAR without jumping-off CRPS. Panels B to E show the ratios of the CRPS of the soft conditioned BVAR without jumping-off to the raw BVAR without jumping-off. Numbers in bold imply that the soft conditioned BVAR without jumping-off improves over the raw BVAR without jumping-off. Asterisks *, ${ }^{* *}$ and ${ }^{* * *}$ imply statistical significance of a two-sided Diebold and Mariano (1995) test of equal predictive ability at the 10\%, 5\%, 1\% level. The variance is estimated using Bartlett kernel weights with bandwidth equal to the forecast horizon $h$. The evaluation sample size is $P=72$ quarters. The columns $h=4^{*}$ and $h=8^{*}$ show the results for the one- and two-year-ahead, year-on-year predictions, respectively. 


\section{C.2 TVP-VAR Results}

Table C.1: Entropic tilting versus TVP-VAR forecasts: RMSFE

\begin{tabular}{|c|c|c|c|c|c|c|c|c|c|c|}
\hline \multirow[t]{2}{*}{ Forecast horizon } & $h=1$ & $h=2$ & $h=3$ & $h=4$ & $h=5$ & $h=6$ & $h=7$ & $h=8$ & $h=4^{*}$ & $h=8^{*}$ \\
\hline & \multicolumn{10}{|c|}{ Panel A. TVP-VAR } \\
\hline GDP & 0.51 & 0.57 & 0.59 & 0.60 & 0.61 & 0.61 & 0.62 & 0.61 & 1.60 & 2.11 \\
\hline HICP & 0.26 & 0.28 & 0.32 & 0.35 & 0.37 & 0.39 & 0.41 & 0.43 & 1.03 & 1.50 \\
\hline Unemployment & 0.26 & 0.40 & 0.56 & 0.73 & 0.89 & 1.05 & 1.20 & 1.34 & $N / A$ & $N / A$ \\
\hline \multirow[t]{2}{*}{ Euribor } & 0.38 & 0.78 & 1.17 & 1.55 & 1.93 & 2.27 & 2.60 & 2.89 & $N / A$ & $N / A$ \\
\hline & \multicolumn{10}{|c|}{ Panel B. Tilting GDP } \\
\hline GDP & $0.86^{* *}$ & $0.89^{* *}$ & $0.92^{* *}$ & 0.93 & 0.94 & 0.94 & 0.93 & $0.95^{* *}$ & $0.86^{* *}$ & 0.91 \\
\hline HICP & 1.00 & 1.00 & $1.02^{*}$ & 1.01 & 1.02 & 1.02 & 1.01 & 1.01 & 1.01 & 1.02 \\
\hline Unemployment & $0.97^{*}$ & 0.93 & 0.92 & 0.91 & 0.91 & 0.92 & 0.92 & 0.92 & $N / A$ & $N / A$ \\
\hline \multirow[t]{2}{*}{ Euribor } & 0.99 & 0.98 & 0.98 & 0.97 & 0.97 & 0.97 & 0.97 & 0.97 & $N / A$ & $N / A$ \\
\hline & \multicolumn{10}{|c|}{ Panel C. Tilting HICP } \\
\hline GDP & 0.99 & 0.98 & 0.98 & 0.99 & 0.99 & 1.01 & 1.01 & 1.01 & 0.97 & 0.99 \\
\hline HICP & $0.90^{*}$ & 0.93 & 0.85 & 0.80 & 0.77 & 0.74 & 0.70 & $0.66^{*}$ & 0.81 & 0.63 \\
\hline Unemployment & 0.99 & 0.97 & 0.97 & 0.97 & 0.97 & 0.98 & 0.98 & 0.99 & $N / A$ & $N / A$ \\
\hline \multirow[t]{2}{*}{ Euribor } & 0.98 & $0.93^{*}$ & $0.89^{* *}$ & $0.87^{* *}$ & $0.86^{* *}$ & $0.84^{* *}$ & $0.84^{* *}$ & $0.84^{* *}$ & $N / A$ & $N / A$ \\
\hline & \multicolumn{10}{|c|}{ Panel D. Tilting Unemployment } \\
\hline GDP & 0.98 & 0.99 & 1.01 & 0.99 & 1.00 & 1.01 & 0.99 & 1.01 & 0.99 & 0.98 \\
\hline HICP & 1.00 & 1.01 & 1.02 & 1.02 & 1.01 & 1.01 & 1.02 & 1.01 & 1.02 & 1.01 \\
\hline Unemployment & 0.98 & 0.98 & 0.98 & 0.99 & 0.98 & 0.98 & 0.96 & 0.95 & $N / A$ & $N / A$ \\
\hline \multirow[t]{2}{*}{ Euribor } & 1.09 & 1.10 & 1.06 & 1.03 & 1.01 & 0.99 & 0.98 & 0.97 & $N / A$ & $N / A$ \\
\hline & \multicolumn{10}{|c|}{ Panel E. Tilting GDP, HICP and Unemployment } \\
\hline GDP & $0.87^{* *}$ & $0.88^{* *}$ & $0.93^{*}$ & 0.95 & 0.95 & 0.96 & 0.93 & 0.99 & $0.86^{* *}$ & 0.91 \\
\hline $\mathrm{HICP}$ & $0.89^{*}$ & 0.93 & 0.86 & 0.79 & 0.76 & 0.73 & 0.69 & $0.67^{*}$ & 0.81 & 0.63 \\
\hline Unemployment & 0.98 & 0.97 & 0.98 & 0.99 & 0.98 & 0.97 & 0.96 & 0.95 & $N / A$ & $N / A$ \\
\hline Euribor & 1.06 & 0.97 & $0.89^{*}$ & $0.83^{* *}$ & $0.80^{* *}$ & $0.78^{*}$ & $0.77^{*}$ & $0.77^{* *}$ & $N / A$ & $N / A$ \\
\hline
\end{tabular}

Note: Panel A displays the raw TVP-VAR RMSFE. Panels B to E show the ratios of the RMSFE of the entropic tilted TVP-VAR to the raw TVP-VAR. Numbers in bold imply that the tilted TVP-VAR improves over the raw TVP-VAR. Asterisks *,** and ${ }^{* * *}$ imply statistical significance of a two-sided Diebold and Mariano (1995) test of equal predictive ability at the $10 \%, 5 \%, 1 \%$ level. The variance is estimated using Bartlett kernel weights with bandwidth equal to the forecast horizon $h$. The evaluation sample size is $P=72$ quarters. The columns $h=4^{*}$ and $h=8^{*}$ show the results for the one- and two-year-ahead, year-on-year predictions, respectively. 
Table C.2: Entropic tilting versus TVP-VAR forecasts: CRPS

\begin{tabular}{|c|c|c|c|c|c|c|c|c|c|c|}
\hline \multirow[t]{3}{*}{ Forecast horizon } & $h=1$ & $h=2$ & $h=3$ & $h=4$ & $h=5$ & $h=6$ & $h=7$ & $h=8$ & $h=4^{*}$ & $h=8^{*}$ \\
\hline & \multicolumn{10}{|c|}{ Panel A. TVP-VAR } \\
\hline & 0.24 & 0.28 & 0.29 & 0.30 & 0.31 & 0.31 & 0.31 & 0.31 & 0.83 & 1.16 \\
\hline HICP & 0.15 & 0.15 & 0.17 & 0.19 & 0.20 & 0.21 & 0.23 & 0.24 & 0.54 & 0.80 \\
\hline Unemployment & 0.17 & 0.23 & 0.31 & 0.41 & 0.52 & 0.62 & 0.72 & 0.81 & $N / A$ & $N / A$ \\
\hline \multirow[t]{2}{*}{ Euribor } & 0.18 & 0.38 & 0.59 & 0.83 & 1.06 & 1.28 & 1.49 & 1.69 & $N / A$ & $N / A$ \\
\hline & \multicolumn{10}{|c|}{ Panel B. Tilting GDP } \\
\hline GDP & $0.85^{* * *}$ & $0.86^{* *}$ & $0.88^{*}$ & $0.88^{*}$ & $0.88^{*}$ & 0.88 & $0.87^{*}$ & $0.90^{* * *}$ & $0.86^{*}$ & 0.88 \\
\hline HICP & 1.00 & 1.00 & 1.02 & 1.01 & 1.02 & 1.02 & 1.02 & 1.00 & 1.02 & 1.02 \\
\hline Unemployment & 0.98 & 0.96 & 0.95 & 0.94 & 0.94 & 0.94 & 0.94 & 0.94 & $N / A$ & $N / A$ \\
\hline \multirow[t]{2}{*}{ Euribor } & 0.99 & 0.99 & 0.98 & 0.97 & 0.97 & 0.97 & 0.96 & 0.96 & $N / A$ & $N / A$ \\
\hline & \multicolumn{10}{|c|}{ Panel C. Tilting HICP } \\
\hline GDP & 0.99 & 0.99 & 0.99 & 0.99 & 1.00 & 1.02 & 1.01 & 1.02 & 0.99 & 1.01 \\
\hline HICP & 0.92 & 0.99 & 0.91 & 0.87 & 0.83 & 0.79 & 0.72 & $0.67^{*}$ & 0.92 & 0.70 \\
\hline Unemployment & 0.99 & 0.97 & 0.98 & 0.98 & 0.98 & 0.98 & 0.99 & 0.99 & $N / A$ & $N / A$ \\
\hline \multirow[t]{2}{*}{ Euribor } & 0.98 & $0.94^{*}$ & $0.90^{*}$ & $0.88^{*}$ & $0.86^{*}$ & $0.85^{*}$ & $0.84^{*}$ & $0.84^{*}$ & $N / A$ & $N / A$ \\
\hline & \multicolumn{10}{|c|}{ Panel D. Tilting Unemployment } \\
\hline GDP & 0.99 & 1.00 & 1.01 & $0.97^{*}$ & 0.98 & 1.00 & 0.98 & 0.99 & 1.01 & 0.97 \\
\hline $\mathrm{HICP}$ & 1.00 & 1.01 & 1.02 & 1.01 & 1.00 & 1.01 & 1.01 & 1.00 & 1.02 & 1.01 \\
\hline Unemployment & 0.98 & 1.01 & 1.02 & 1.02 & 1.01 & 0.99 & 0.98 & 0.97 & $N / A$ & $N / A$ \\
\hline \multirow[t]{2}{*}{ Euribor } & 1.07 & 1.09 & $1.06^{*}$ & 1.03 & 1.00 & 0.97 & 0.96 & 0.95 & $N / A$ & $N / A$ \\
\hline & \multicolumn{10}{|c|}{ Panel E. Tilting GDP, HICP and Unemployment } \\
\hline GDP & $0.87^{* *}$ & $0.87^{* *}$ & 0.90 & $0.89^{*}$ & 0.90 & 0.90 & $0.86^{*}$ & $0.95^{* *}$ & $0.87^{*}$ & 0.89 \\
\hline $\mathrm{HICP}$ & 0.92 & 1.00 & 0.93 & 0.86 & 0.82 & 0.79 & 0.72 & $0.68^{*}$ & 0.92 & 0.71 \\
\hline Unemployment & 0.97 & 1.00 & 1.02 & 1.01 & 1.00 & 0.99 & 0.98 & 0.97 & $N / A$ & $N / A$ \\
\hline Euribor & 1.04 & 0.98 & 0.90 & $0.85^{*}$ & $0.81^{*}$ & $0.78^{*}$ & $0.77^{* *}$ & $0.76^{* *}$ & $N / A$ & $N / A$ \\
\hline
\end{tabular}

Note: Panel A displays the raw TVP-VAR CRPS. Panels B to E show the ratios of the CRPS of the entropic tilted TVP-VAR to the raw TVP-VAR. Numbers in bold imply that the tilted TVP-VAR improves over the raw TVP-VAR. Asterisks *, ** and ${ }^{* * *}$ imply statistical significance of a two-sided Diebold and Mariano (1995) test of equal predictive ability at the $10 \%, 5 \%, 1 \%$ level. The variance is estimated using Bartlett kernel weights with bandwidth equal to the forecast horizon $h$. The evaluation sample size is $P=72$ quarters. The columns $h=4^{*}$ and $h=8^{*}$ show the results for the one- and two-year-ahead, year-on-year predictions, respectively. 
Table C.3: Soft Conditioning versus TVP-VAR forecasts: RMSFE

\begin{tabular}{|c|c|c|c|c|c|c|c|c|c|c|}
\hline \multirow[t]{2}{*}{ Forecast horizon } & $h=1$ & $h=2$ & $h=3$ & $h=4$ & $h=5$ & $h=6$ & $h=7$ & $h=8$ & $h=4 *$ & $h=8 *$ \\
\hline & \multicolumn{10}{|c|}{ Panel A. TVP-VAR } \\
\hline GDP & 0.51 & 0.57 & 0.59 & 0.60 & 0.61 & 0.61 & 0.62 & 0.61 & 1.60 & 2.11 \\
\hline HICP & 0.26 & 0.28 & 0.32 & 0.35 & 0.37 & 0.39 & 0.41 & 0.43 & 1.03 & 1.50 \\
\hline Unemployment & 0.26 & 0.40 & 0.56 & 0.73 & 0.89 & 1.05 & 1.20 & 1.34 & $N / A$ & $N / A$ \\
\hline \multirow[t]{2}{*}{ Euribor } & 0.38 & 0.78 & 1.17 & 1.55 & 1.93 & 2.27 & 2.60 & 2.89 & $N / A$ & $N / A$ \\
\hline & \multicolumn{10}{|c|}{ Panel B. Soft Conditioning GDP } \\
\hline GDP & $0.92^{* *}$ & $0.94^{* * *}$ & $0.95^{* *}$ & $0.96^{*}$ & 0.96 & 0.96 & 0.95 & $0.96^{* *}$ & $0.92^{* * *}$ & 0.93 \\
\hline HICP & 1.00 & 1.00 & 1.00 & 1.00 & 1.01 & 1.01 & 1.01 & 1.01 & 1.00 & 1.01 \\
\hline Unemployment & 0.98 & 0.97 & 0.96 & 0.96 & 0.96 & 0.96 & 0.96 & 0.96 & $N / A$ & $N / A$ \\
\hline \multirow[t]{2}{*}{ Euribor } & 1.00 & 0.99 & 0.99 & 0.98 & 0.98 & 0.98 & 0.98 & 0.98 & $N / A$ & $N / A$ \\
\hline & \multicolumn{10}{|c|}{ Panel C. Soft Conditioning HICP } \\
\hline GDP & 0.99 & 0.98 & 0.98 & 0.98 & 0.99 & 1.00 & 1.00 & 1.01 & 0.98 & 0.99 \\
\hline HICP & $0.93^{*}$ & 0.92 & 0.85 & 0.78 & 0.74 & $0.70^{*}$ & $0.67^{*}$ & $0.65^{* *}$ & 0.81 & $0.60^{*}$ \\
\hline Unemployment & 0.99 & 0.98 & 0.97 & 0.97 & 0.97 & 0.98 & 0.98 & 0.99 & $N / A$ & $N / A$ \\
\hline \multirow[t]{2}{*}{ Euribor } & 0.98 & $0.94^{* *}$ & $0.91^{* *}$ & $0.88^{* *}$ & $0.87^{* *}$ & $0.86^{* *}$ & $0.85^{* *}$ & $0.85^{* *}$ & $N / A$ & $N / A$ \\
\hline & \multicolumn{10}{|c|}{ Panel D. Soft Conditioning Unemployment } \\
\hline GDP & 0.99 & 1.00 & 1.00 & 0.99 & $0.99^{*}$ & 0.99 & $0.98^{*}$ & $0.98^{*}$ & 1.00 & $0.98^{*}$ \\
\hline HICP & 1.00 & 1.00 & 1.01 & 1.01 & 1.01 & 1.02 & 1.02 & 1.02 & 1.01 & 1.02 \\
\hline Unemployment & 0.99 & 0.99 & 0.99 & 0.99 & 0.99 & 0.99 & 0.99 & 0.99 & $N / A$ & $N / A$ \\
\hline \multirow[t]{2}{*}{ Euribor } & 1.04 & 1.05 & $1.04^{*}$ & $1.03^{*}$ & $1.02^{* *}$ & 1.01 & 1.01 & 1.00 & $N / A$ & $N / A$ \\
\hline & \multicolumn{10}{|c|}{ Panel E. Soft Conditioning GDP, HICP and Unemployment } \\
\hline GDP & $0.91^{* *}$ & $0.92^{* * *}$ & $0.95^{* * *}$ & $0.94^{* * *}$ & $0.95^{* *}$ & 0.96 & $0.95^{*}$ & $0.97^{* * *}$ & $0.91^{* *}$ & $0.93^{* *}$ \\
\hline HICP & $0.93^{*}$ & 0.92 & 0.85 & 0.78 & $0.73^{*}$ & $0.70^{*}$ & $0.68^{*}$ & $0.66^{* *}$ & 0.81 & $0.61^{*}$ \\
\hline Unemployment & 0.98 & 0.95 & 0.95 & 0.95 & 0.95 & 0.96 & 0.96 & 0.96 & $N / A$ & $N / A$ \\
\hline Euribor & 1.00 & 0.96 & $0.92^{* *}$ & $0.88^{* *}$ & $0.86^{* *}$ & $0.85^{* *}$ & $0.84^{* *}$ & $0.83^{* *}$ & $N / A$ & $N / A$ \\
\hline
\end{tabular}

Note: Panel A displays the raw TVP-VAR RMSFE. Panels B to E show the ratios of the RMSFE of the soft conditioned TVP-VAR to the raw TVP-VAR. Numbers in bold imply that the soft conditioned TVP-VAR improves over the raw TVP-VAR. Asterisks *, ** and ${ }^{* * *}$ imply statistical significance of a two-sided Diebold and Mariano (1995) test of equal predictive ability at the $10 \%$, $5 \%, 1 \%$ level. The variance is estimated using Bartlett kernel weights with bandwidth equal to the forecast horizon $h$. The evaluation sample size is $P=72$ quarters. The columns $h=4^{*}$ and $h=8^{*}$ show the results for the one- and two-year-ahead, year-on-year predictions, respectively. 
Table C.4: Soft Conditioning versus TVP-VAR forecasts: CRPS

\begin{tabular}{|c|c|c|c|c|c|c|c|c|c|c|}
\hline \multirow[t]{2}{*}{ Forecast horizon } & $h=1$ & $h=2$ & $h=3$ & $h=4$ & $h=5$ & $h=6$ & $h=7$ & $h=8$ & $h=4 *$ & $h=8 *$ \\
\hline & \multicolumn{10}{|c|}{ Panel A. TVP-VAR } \\
\hline GDP & 0.24 & 0.28 & 0.29 & 0.30 & 0.31 & 0.31 & 0.31 & 0.31 & 0.83 & 1.16 \\
\hline HICP & 0.15 & 0.15 & 0.17 & 0.19 & 0.20 & 0.21 & 0.23 & 0.24 & 0.54 & 0.80 \\
\hline Unemployment & 0.17 & 0.23 & 0.31 & 0.41 & 0.52 & 0.62 & 0.72 & 0.81 & $N / A$ & $N / A$ \\
\hline \multirow[t]{2}{*}{ Euribor } & 0.18 & 0.38 & 0.59 & 0.83 & 1.06 & 1.28 & 1.49 & 1.69 & $N / A$ & $N / A$ \\
\hline & \multicolumn{10}{|c|}{ Panel B. Soft Conditioning GDP } \\
\hline GDP & $0.91^{* * *}$ & $0.92^{* * *}$ & $0.93^{* *}$ & $0.93^{* *}$ & $0.93^{*}$ & $0.92^{*}$ & $0.91^{* *}$ & $0.94^{* * *}$ & $0.94^{*}$ & 0.94 \\
\hline HICP & 1.00 & 1.00 & 1.00 & 1.01 & 1.01 & 1.02 & 1.02 & 1.01 & 1.00 & 1.02 \\
\hline Unemployment & 1.00 & 1.00 & 0.99 & 0.99 & 0.99 & 0.99 & 0.99 & 0.99 & $N / A$ & $N / A$ \\
\hline \multirow[t]{2}{*}{ Euribor } & 0.99 & 1.00 & 0.99 & 0.98 & 0.98 & 0.98 & 0.97 & 0.97 & $N / A$ & $N / A$ \\
\hline & \multicolumn{10}{|c|}{ Panel C. Soft Conditioning HICP } \\
\hline GDP & 0.99 & 0.98 & 0.99 & 0.99 & 0.99 & 1.00 & 1.00 & 1.01 & 0.99 & 1.00 \\
\hline HICP & 0.96 & 0.98 & 0.90 & 0.84 & 0.78 & $0.75^{*}$ & $0.70^{*}$ & $0.67^{* *}$ & 0.89 & $0.65^{*}$ \\
\hline Unemployment & 0.99 & 0.98 & 0.98 & 0.98 & 0.98 & 0.98 & 0.99 & 0.99 & $N / A$ & $N / A$ \\
\hline \multirow[t]{2}{*}{ Euribor } & 0.98 & $0.94^{* *}$ & $0.90^{* *}$ & $0.89^{* *}$ & $0.87^{* *}$ & $0.86^{* *}$ & $0.85^{* *}$ & $0.85^{* *}$ & $N / A$ & $N / A$ \\
\hline & \multicolumn{10}{|c|}{ Panel D. Soft Conditioning Unemployment } \\
\hline GDP & $0.99^{*}$ & 0.99 & 1.00 & $0.98^{* *}$ & $0.97^{* *}$ & $0.98^{*}$ & $0.97^{* *}$ & $0.97^{* *}$ & 1.01 & $0.97^{*}$ \\
\hline HICP & 1.00 & 1.00 & 1.00 & 1.00 & 1.01 & 1.01 & 1.02 & 1.02 & 1.00 & 1.02 \\
\hline Unemployment & $1.01^{*}$ & $1.01^{*}$ & 1.01 & 1.01 & 1.02 & 1.03 & 1.03 & 1.03 & $N / A$ & $N / A$ \\
\hline \multirow[t]{2}{*}{ Euribor } & 1.03 & 1.05 & 1.04 & $1.03^{* *}$ & 1.02 & 1.01 & 1.00 & 0.99 & $N / A$ & $N / A$ \\
\hline & \multicolumn{10}{|c|}{ Panel E. Soft Conditioning GDP, HICP and Unemployment } \\
\hline GDP & $0.91^{* * *}$ & $0.91^{* * *}$ & $0.93^{* * *}$ & $0.92^{* * *}$ & $0.93^{* * *}$ & $0.93^{* *}$ & $0.92^{* * *}$ & $0.94^{* * *}$ & $0.94^{*}$ & 0.95 \\
\hline HICP & 0.96 & 0.98 & 0.91 & 0.84 & $0.78^{*}$ & $0.75^{*}$ & $0.72^{*}$ & $0.68^{* *}$ & 0.89 & $0.67^{*}$ \\
\hline Unemployment & 1.00 & 0.99 & 0.99 & 1.00 & 1.01 & 1.01 & 1.02 & 1.02 & $N / A$ & $N / A$ \\
\hline Euribor & 0.99 & 0.96 & $0.92^{* *}$ & $0.89^{* *}$ & $0.87^{* *}$ & $0.85^{* *}$ & $0.84^{* * *}$ & $0.83^{* * *}$ & $N / A$ & $N / A$ \\
\hline
\end{tabular}

Note: Panel A displays the raw TVP-VAR CRPS. Panels B to E show the ratios of the CRPS of the soft conditioned TVP-VAR to the raw TVP-VAR. Numbers in bold imply that the soft conditioned TVP-VAR improves over the raw TVP-VAR. Asterisks *, ** and ${ }^{* * *}$ imply statistical significance of a two-sided Diebold and Mariano (1995) test of equal predictive ability at the $10 \%$, $5 \%, 1 \%$ level. The variance is estimated using Bartlett kernel weights with bandwidth equal to the forecast horizon $h$. The evaluation sample size is $P=72$ quarters. The columns $h=4^{*}$ and $h=8^{*}$ show the results for the one- and two-year-ahead, year-on-year predictions, respectively. 
Table C.5: Entropic tilting versus large BVAR forecasts: RMSFE

\begin{tabular}{|c|c|c|c|c|c|c|c|c|c|c|}
\hline \multirow[t]{2}{*}{ Forecast horizon } & $h=1$ & $h=2$ & $h=3$ & $h=4$ & $h=5$ & $h=6$ & $h=7$ & $h=8$ & $h=4^{*}$ & $h=8^{*}$ \\
\hline & \multicolumn{10}{|c|}{ Panel A. TVP-VAR } \\
\hline GDP & 0.47 & 0.55 & 0.59 & 0.60 & 0.60 & 0.59 & 0.59 & 0.57 & 1.55 & 2.01 \\
\hline HICP & 0.30 & 0.31 & 0.33 & 0.35 & 0.36 & 0.36 & 0.37 & 0.37 & 1.12 & 1.34 \\
\hline Unemployment & 0.25 & 0.38 & 0.53 & 0.70 & 0.86 & 1.04 & 1.21 & 1.37 & $N / A$ & $N / A$ \\
\hline \multirow[t]{2}{*}{ Euribor } & 0.38 & 0.69 & 1.03 & 1.38 & 1.73 & 2.04 & 2.33 & 2.56 & $N / A$ & $N / A$ \\
\hline & \multicolumn{10}{|c|}{ Panel B. Tilting GDP } \\
\hline GDP & $0.89^{* * *}$ & $0.92^{* * *}$ & 0.94 & 0.94 & 0.96 & 0.98 & 0.99 & 1.00 & $0.89^{* * *}$ & 0.95 \\
\hline HICP & 1.01 & 1.00 & 0.99 & 0.97 & 0.97 & 0.98 & 0.98 & 0.98 & 0.99 & 0.97 \\
\hline Unemployment & 1.02 & 1.02 & 1.03 & 1.03 & 1.04 & 1.05 & 1.05 & 1.05 & $N / A$ & $N / A$ \\
\hline \multirow[t]{2}{*}{ Euribor } & $0.98^{*}$ & $0.96^{* * *}$ & $0.93^{* * *}$ & $0.92^{* *}$ & $0.91^{*}$ & 0.92 & 0.93 & 0.95 & $N / A$ & $N / A$ \\
\hline & \multicolumn{10}{|c|}{ Panel C. Tilting HICP } \\
\hline GDP & 0.97 & 0.99 & 0.97 & 0.95 & 0.95 & 0.95 & 0.96 & 0.99 & 0.96 & $0.92^{* *}$ \\
\hline $\mathrm{HICP}$ & 0.83 & 0.84 & 0.82 & 0.81 & $0.80^{* *}$ & $0.79^{*}$ & $0.77^{* *}$ & $0.76^{* *}$ & 0.74 & $0.72^{* *}$ \\
\hline Unemployment & 0.98 & 0.99 & 1.00 & 1.00 & 1.01 & 1.01 & 1.01 & 1.01 & $N / A$ & $N / A$ \\
\hline \multirow[t]{2}{*}{ Euribor } & 0.99 & $0.97^{* * *}$ & $0.95^{* *}$ & $0.92^{*}$ & $0.89^{*}$ & $0.87^{*}$ & $0.86^{*}$ & $0.85^{*}$ & $N / A$ & $N / A$ \\
\hline & \multicolumn{10}{|c|}{ Panel D. Tilting Unemployment } \\
\hline GDP & 0.98 & 0.98 & 0.99 & 0.97 & $0.97^{*}$ & 0.98 & 0.98 & 1.02 & 0.99 & 0.96 \\
\hline $\mathrm{HICP}$ & 1.01 & 1.01 & 1.00 & 0.99 & 0.99 & 1.00 & 0.99 & 0.97 & 1.00 & 0.98 \\
\hline Unemployment & 1.03 & 1.04 & 1.03 & 1.02 & 1.02 & 1.01 & 0.98 & 0.93 & $N / A$ & $N / A$ \\
\hline \multirow[t]{2}{*}{ Euribor } & 0.99 & 0.98 & 0.97 & 0.97 & 0.96 & $0.95^{*}$ & $0.93^{* *}$ & $0.92^{* *}$ & $N / A$ & $N / A$ \\
\hline & \multicolumn{10}{|c|}{ Panel E. Tilting GDP, HICP and Unemployment } \\
\hline GDP & $0.90^{* * *}$ & $0.93^{* *}$ & 0.94 & 0.95 & 0.96 & 0.98 & 1.03 & $1.06^{*}$ & $0.89^{* * *}$ & 0.95 \\
\hline HICP & 0.83 & 0.83 & $0.81^{*}$ & $0.81^{*}$ & $0.79^{* *}$ & $0.80^{* *}$ & $0.79^{* *}$ & $0.74^{* *}$ & 0.74 & $0.72^{* *}$ \\
\hline Unemployment & 1.02 & 1.04 & 1.03 & 1.02 & 1.01 & 0.98 & 0.95 & 0.93 & $N / A$ & $N / A$ \\
\hline Euribor & 1.01 & $0.95^{* * *}$ & $0.90^{* * *}$ & $0.87^{* *}$ & $0.86^{* *}$ & $0.83^{* *}$ & $0.82^{* *}$ & $0.81^{* *}$ & $N / A$ & $N / A$ \\
\hline
\end{tabular}

Note: Panel A displays the raw large BVAR RMSFE. Panels B to E show the ratios of the RMSFE of the entropic tilted large BVAR to the raw large BVAR. Numbers in bold imply that the tilted large BVAR improves over the raw large BVAR. Asterisks * , ** and *** imply statistical significance of a two-sided Diebold and Mariano (1995) test of equal predictive ability at the $10 \%, 5 \%, 1 \%$ level. The variance is estimated using Bartlett kernel weights with bandwidth equal to the forecast horizon $h$. The evaluation sample size is $P=72$ quarters. The columns $h=4^{*}$ and $h=8^{*}$ show the results for the one- and two-year-ahead, year-on-year predictions, respectively. 
Table C.6: Entropic tilting versus large BVAR forecasts: CRPS

\begin{tabular}{|c|c|c|c|c|c|c|c|c|c|c|}
\hline \multirow[t]{2}{*}{ Forecast horizon } & $h=1$ & $h=2$ & $h=3$ & $h=4$ & $h=5$ & $h=6$ & $h=7$ & $h=8$ & $h=4^{*}$ & $h=8^{*}$ \\
\hline & \multicolumn{10}{|c|}{ Panel A. TVP-VAR } \\
\hline GDP & 0.24 & 0.28 & 0.29 & 0.31 & 0.32 & 0.34 & 0.35 & 0.35 & 0.80 & 1.15 \\
\hline HICP & 0.16 & 0.17 & 0.19 & 0.20 & 0.21 & 0.21 & 0.23 & 0.23 & 0.62 & 0.81 \\
\hline Unemployment & 0.15 & 0.21 & 0.30 & 0.40 & 0.50 & 0.61 & 0.71 & 0.81 & $N / A$ & $N / A$ \\
\hline \multirow[t]{2}{*}{ Euribor } & 0.18 & 0.35 & 0.54 & 0.75 & 0.95 & 1.13 & 1.29 & 1.42 & $N / A$ & $N / A$ \\
\hline & \multicolumn{10}{|c|}{ Panel B. Tilting GDP } \\
\hline GDP & $0.85^{* * *}$ & $0.84^{* * *}$ & 0.87 & $0.85^{* *}$ & 0.84 & $0.82^{*}$ & $0.81^{* *}$ & $0.85^{* *}$ & $0.87^{* *}$ & 0.91 \\
\hline $\mathrm{HICP}$ & 1.00 & 0.99 & 0.98 & $0.96^{*}$ & 0.95 & 0.95 & 0.95 & 0.94 & 0.98 & $0.93^{*}$ \\
\hline Unemployment & 1.02 & 1.02 & 1.01 & 1.00 & 1.00 & 1.01 & 1.02 & 1.03 & $N / A$ & $N / A$ \\
\hline \multirow[t]{2}{*}{ Euribor } & $0.98^{* * *}$ & $0.94^{* * *}$ & $0.91^{* * *}$ & $0.90^{* * *}$ & $0.90^{* *}$ & $0.90^{*}$ & 0.92 & 0.93 & $N / A$ & $N / A$ \\
\hline & \multicolumn{10}{|c|}{ Panel C. Tilting HICP } \\
\hline GDP & 0.99 & 1.00 & 0.99 & 0.95 & 0.94 & 0.92 & 0.92 & 0.94 & 0.97 & 0.90 \\
\hline HICP & $0.86^{* *}$ & 0.86 & $0.83^{*}$ & $0.81^{*}$ & $0.80^{* *}$ & $0.77^{* *}$ & $0.73^{* *}$ & $0.71^{* * *}$ & 0.79 & $0.71^{* *}$ \\
\hline Unemployment & 0.99 & 0.99 & 0.99 & 0.98 & 0.97 & 0.96 & 0.97 & 0.97 & $N / A$ & $N / A$ \\
\hline \multirow[t]{2}{*}{ Euribor } & 0.99 & $0.97^{* * *}$ & $0.95^{* *}$ & $0.92^{*}$ & $0.91^{* *}$ & $0.89 *$ & $0.88^{* *}$ & $0.88^{*}$ & $N / A$ & $N / A$ \\
\hline & \multicolumn{10}{|c|}{ Panel D. Tilting Unemployment } \\
\hline GDP & 0.99 & 0.99 & 0.98 & $0.95^{* *}$ & $0.93^{* * *}$ & $0.94^{* *}$ & $0.93^{* *}$ & 0.95 & 1.01 & $0.93^{* *}$ \\
\hline HICP & 1.01 & 1.00 & 1.00 & 0.98 & 0.98 & 0.98 & 0.96 & 0.95 & 1.00 & 0.95 \\
\hline Unemployment & 1.05 & 1.06 & 1.03 & 1.02 & 0.99 & 0.96 & 0.95 & 0.96 & $N / A$ & $N / A$ \\
\hline \multirow[t]{2}{*}{ Euribor } & 1.01 & 0.99 & 0.99 & 0.99 & 0.97 & $0.95^{*}$ & $0.93^{* *}$ & $0.92^{* * *}$ & $N / A$ & $N / A$ \\
\hline & \multicolumn{10}{|c|}{ Panel E. Tilting GDP, HICP and Unemployment } \\
\hline GDP & $0.86^{* * *}$ & $0.85^{* * *}$ & 0.87 & $0.87^{*}$ & $0.84^{*}$ & $0.83^{*}$ & 0.85 & 0.88 & $0.86^{* *}$ & 0.91 \\
\hline HICP & $0.86^{*}$ & 0.86 & $0.82^{*}$ & $0.81^{*}$ & $0.79^{* *}$ & $0.77^{* *}$ & $0.74^{* *}$ & $0.70^{* * *}$ & 0.79 & $0.71^{* *}$ \\
\hline Unemployment & 1.04 & 1.07 & 1.05 & 1.03 & 1.00 & 0.97 & 0.95 & 0.96 & $N / A$ & $N / A$ \\
\hline Euribor & 1.02 & $0.93^{* * *}$ & $0.88^{* * *}$ & $0.86^{* * *}$ & $0.84^{* * *}$ & $0.83^{* * *}$ & $0.82^{* * *}$ & $0.81^{* * *}$ & $N / A$ & $N / A$ \\
\hline
\end{tabular}

Note: Panel A displays the raw large BVAR CRPS. Panels B to E show the ratios of the CRPS of the entropic tilted large BVAR to the raw large BVAR. Numbers in bold imply that the tilted large BVAR improves over the raw large BVAR. Asterisks * ${ }^{* *}$ and ${ }^{* *}$ imply statistical significance of a two-sided Diebold and Mariano (1995) test of equal predictive ability at the $10 \%, 5 \%, 1 \%$ level. The variance is estimated using Bartlett kernel weights with bandwidth equal to the forecast horizon $h$. The evaluation sample size is $P=72$ quarters. The columns $h=4^{*}$ and $h=8^{*}$ show the results for the one- and two-year-ahead, year-on-year predictions, respectively. 


\section{C.3 Large BVAR Results}

Table C.7: Soft Conditioning versus large BVAR forecasts: RMSFE

\begin{tabular}{|c|c|c|c|c|c|c|c|c|c|c|}
\hline \multirow[t]{2}{*}{ Forecast horizon } & $h=1$ & $h=2$ & $h=3$ & $h=4$ & $h=5$ & $h=6$ & $h=7$ & $h=8$ & $h=4 *$ & $h=8 *$ \\
\hline & \multicolumn{10}{|c|}{ Panel A. BVAR } \\
\hline GDP & 0.47 & 0.55 & 0.59 & 0.60 & 0.60 & 0.59 & 0.59 & 0.57 & 1.55 & 2.01 \\
\hline HICP & 0.30 & 0.31 & 0.33 & 0.35 & 0.36 & 0.36 & 0.37 & 0.37 & 1.12 & 1.34 \\
\hline Unemployment & 0.25 & 0.38 & 0.53 & 0.70 & 0.86 & 1.04 & 1.21 & 1.37 & $N / A$ & $N / A$ \\
\hline \multirow[t]{2}{*}{ Euribor } & 0.38 & 0.69 & 1.03 & 1.38 & 1.73 & 2.04 & 2.33 & 2.56 & $N / A$ & $N / A$ \\
\hline & \multicolumn{10}{|c|}{ Panel B. Soft Conditioning GDP } \\
\hline GDP & $0.92^{* * *}$ & $0.93^{* * *}$ & 0.93 & 0.93 & 0.95 & 0.97 & 0.97 & 0.99 & $0.90^{* * *}$ & 0.93 \\
\hline HICP & 1.01 & 1.00 & 0.99 & 0.98 & 0.98 & 0.98 & 0.98 & 0.98 & 0.99 & 0.98 \\
\hline Unemployment & 1.01 & 1.01 & 1.01 & 1.02 & 1.02 & 1.03 & 1.03 & 1.03 & $N / A$ & $N / A$ \\
\hline \multirow[t]{2}{*}{ Euribor } & 0.99 & $0.96^{* * *}$ & $0.94^{* * *}$ & $0.92^{* *}$ & $0.92^{* *}$ & 0.92 & 0.93 & 0.94 & $N / A$ & $N / A$ \\
\hline & \multicolumn{10}{|c|}{ Panel C. Soft Conditioning HICP } \\
\hline GDP & 0.98 & 0.97 & 0.96 & 0.95 & 0.95 & 0.95 & 0.96 & 0.98 & $0.96^{*}$ & 0.92 \\
\hline HICP & $0.90^{*}$ & $0.90^{*}$ & $0.86^{* *}$ & $0.83^{* *}$ & $0.82^{* *}$ & $0.80^{* *}$ & $0.78^{* *}$ & $0.77^{* *}$ & $0.82^{* *}$ & $0.74^{* *}$ \\
\hline Unemployment & 0.99 & 0.99 & 0.98 & 0.98 & 0.99 & 0.99 & 0.99 & 0.99 & $N / A$ & $N / A$ \\
\hline \multirow[t]{2}{*}{ Euribor } & 0.99 & $0.97^{* *}$ & $0.94^{* * *}$ & $0.91^{* *}$ & $0.89^{* *}$ & $0.87^{* *}$ & $0.86^{* *}$ & $0.86^{* *}$ & $N / A$ & $N / A$ \\
\hline & \multicolumn{10}{|c|}{ Panel D. Soft Conditioning Unemployment } \\
\hline GDP & 0.99 & 0.99 & 0.98 & $0.96^{* *}$ & $0.94^{* *}$ & $0.94^{* * *}$ & $0.94^{* * *}$ & $0.97^{* *}$ & 0.99 & $0.92^{* * *}$ \\
\hline HICP & 1.00 & 1.00 & 1.00 & 1.00 & 0.99 & 1.00 & 0.99 & 0.98 & 1.00 & 0.99 \\
\hline Unemployment & 1.01 & 1.01 & 0.99 & 0.98 & 0.98 & 0.97 & 0.95 & $0.91^{* *}$ & $N / A$ & $N / A$ \\
\hline \multirow[t]{2}{*}{ Euribor } & 1.00 & $0.99^{*}$ & $0.98^{* *}$ & 0.98 & 0.97 & $0.96^{*}$ & $0.94^{* *}$ & $0.93^{* * *}$ & $N / A$ & $N / A$ \\
\hline & \multicolumn{10}{|c|}{ Panel E. Soft Conditioning GDP, HICP and Unemployment } \\
\hline GDP & $0.92^{* * *}$ & $0.92^{* * *}$ & $0.92 *$ & 0.94 & 0.94 & 0.96 & 0.97 & 0.99 & $0.89^{* * *}$ & 0.92 \\
\hline $\mathrm{HICP}$ & $0.90^{*}$ & $0.90^{*}$ & $0.87^{* *}$ & $0.85^{* *}$ & $0.84^{* *}$ & $0.81^{* *}$ & $0.82^{* * *}$ & $0.76^{* * *}$ & $0.83^{* *}$ & $0.75^{* * *}$ \\
\hline Unemployment & 1.00 & 1.00 & 0.98 & 0.97 & 0.97 & 0.96 & 0.95 & 0.94 & $N / A$ & $N / A$ \\
\hline Euribor & 0.99 & $0.93^{* * *}$ & $0.89^{* * *}$ & $0.86^{* * *}$ & $0.84^{* *}$ & $0.83^{* *}$ & $0.82^{* *}$ & $0.82^{* *}$ & $N / A$ & $N / A$ \\
\hline
\end{tabular}

Note: Panel A displays the raw large BVAR RMSFE. Panels B to E show the ratios of the RMSFE of the soft conditioned large BVAR to the raw large BVAR. Numbers in bold imply that the soft conditioned large BVAR improves over the raw large BVAR. Asterisks *, ** and *** imply statistical significance of a two-sided Diebold and Mariano (1995) test of equal predictive ability at the $10 \%, 5 \%, 1 \%$ level. The variance is estimated using Bartlett kernel weights with bandwidth equal to the forecast horizon $h$. The evaluation sample size is $P=72$ quarters. The columns $h=4^{*}$ and $h=8^{*}$ show the results for the one- and two-year-ahead, year-on-year predictions, respectively. 
Table C.8: Soft Conditioning versus large BVAR forecasts: CRPS

\begin{tabular}{|c|c|c|c|c|c|c|c|c|c|c|}
\hline \multirow[t]{2}{*}{ Forecast horizon } & $h=1$ & $h=2$ & $h=3$ & $h=4$ & $h=5$ & $h=6$ & $h=7$ & $h=8$ & $h=4 *$ & $h=8 *$ \\
\hline & \multicolumn{10}{|c|}{ Panel A. BVAR } \\
\hline GDP & 0.24 & 0.28 & 0.29 & 0.31 & 0.32 & 0.34 & 0.35 & 0.35 & 0.80 & 1.15 \\
\hline HICP & 0.16 & 0.17 & 0.19 & 0.20 & 0.21 & 0.21 & 0.23 & 0.23 & 0.62 & 0.81 \\
\hline Unemployment & 0.15 & 0.21 & 0.30 & 0.40 & 0.50 & 0.61 & 0.71 & 0.81 & $N / A$ & $N / A$ \\
\hline \multirow[t]{2}{*}{ Euribor } & 0.18 & 0.35 & 0.54 & 0.75 & 0.95 & 1.13 & 1.29 & 1.42 & $N / A$ & $N / A$ \\
\hline & \multicolumn{10}{|c|}{ Panel B. Soft Conditioning GDP } \\
\hline GDP & $0.89^{* * *}$ & $0.87^{* * *}$ & $0.87^{* *}$ & $0.85^{* * *}$ & $0.83^{*}$ & $0.80^{* *}$ & $0.80^{* *}$ & $0.84^{* *}$ & $0.90^{* * *}$ & 0.88 \\
\hline HICP & 1.01 & 1.00 & $0.98^{*}$ & $0.97^{*}$ & $0.96^{*}$ & 0.95 & 0.95 & 0.94 & 0.99 & 0.93 \\
\hline Unemployment & 1.02 & 1.01 & 1.00 & 0.99 & 0.99 & 0.99 & 1.00 & 1.01 & $N / A$ & $N / A$ \\
\hline \multirow[t]{2}{*}{ Euribor } & $0.98^{* * *}$ & $0.95^{* * *}$ & $0.92^{* * *}$ & $0.91^{* * *}$ & $0.90^{* * *}$ & $0.91^{* *}$ & $0.92^{*}$ & 0.93 & $N / A$ & $N / A$ \\
\hline & \multicolumn{10}{|c|}{ Panel C. Soft Conditioning HICP } \\
\hline GDP & 0.99 & 0.98 & 0.96 & 0.94 & 0.93 & 0.92 & $0.91^{*}$ & 0.93 & 0.97 & 0.90 \\
\hline HICP & $0.92^{* * *}$ & $0.92^{* *}$ & $0.87^{* *}$ & $0.84^{* *}$ & $0.82^{* *}$ & $0.79^{* *}$ & $0.74^{* * *}$ & $0.73^{* * *}$ & $0.86^{* *}$ & $0.74^{* *}$ \\
\hline Unemployment & 1.00 & 0.99 & 0.98 & 0.97 & 0.96 & 0.95 & 0.95 & 0.96 & $N / A$ & $N / A$ \\
\hline \multirow[t]{2}{*}{ Euribor } & 0.99 & $0.97^{* * *}$ & $0.95^{* * *}$ & $0.92^{* *}$ & $0.90^{* *}$ & $0.89^{* *}$ & $0.88^{* *}$ & $0.88^{* *}$ & $N / A$ & $N / A$ \\
\hline & \multicolumn{10}{|c|}{ Panel D. Soft Conditioning Unemployment } \\
\hline GDP & 1.00 & 0.99 & 0.97 & $0.93^{* * *}$ & $0.91^{* * *}$ & $0.89^{* * *}$ & $0.89^{* * *}$ & $0.91^{* *}$ & 1.00 & $0.88^{* * *}$ \\
\hline HICP & $1.01^{*}$ & 1.00 & 1.00 & 0.99 & 0.98 & 0.97 & 0.96 & 0.95 & 1.00 & 0.95 \\
\hline Unemployment & 1.02 & 1.01 & 0.98 & 0.96 & 0.94 & $0.92^{* *}$ & $0.90^{*}$ & $0.90^{*}$ & $N / A$ & $N / A$ \\
\hline \multirow[t]{2}{*}{ Euribor } & 1.00 & $0.99^{* *}$ & $0.99^{* *}$ & 0.98 & $0.97^{*}$ & $0.95^{* *}$ & $0.94^{* * *}$ & $0.93^{* * *}$ & $N / A$ & $N / A$ \\
\hline & \multicolumn{10}{|c|}{ Panel E. Soft Conditioning GDP, HICP and Unemployment } \\
\hline GDP & $0.90^{* * *}$ & $0.87^{* * *}$ & $0.87^{* *}$ & $0.86^{* *}$ & $0.83^{* *}$ & $0.81^{* *}$ & $0.78^{* *}$ & $0.82^{* *}$ & $0.91^{* * *}$ & 0.88 \\
\hline HICP & $0.93^{* *}$ & $0.92^{* *}$ & $0.89^{* *}$ & $0.87^{* * *}$ & $0.84^{* *}$ & $0.80^{* *}$ & $0.78^{* * *}$ & $0.72^{* * *}$ & $0.88^{* *}$ & $0.76^{* *}$ \\
\hline Unemployment & 1.02 & 1.02 & 0.98 & 0.96 & 0.94 & 0.93 & 0.92 & 0.94 & $N / A$ & $N / A$ \\
\hline Euribor & 0.99 & $0.93^{* * *}$ & $0.88^{* * *}$ & $0.86^{* * *}$ & $0.84^{* * *}$ & $0.84^{* * *}$ & $0.83^{* * *}$ & $0.83^{* * *}$ & $N / A$ & $N / A$ \\
\hline
\end{tabular}

Note: Panel A displays the raw large BVAR CRPS. Panels B to E show the ratios of the CRPS of the soft conditioned large BVAR to the raw large BVAR. Numbers in bold imply that the soft conditioned large BVAR improves over the raw large BVAR. Asterisks *, ${ }^{* *}$ and ${ }^{* * *}$ imply statistical significance of a two-sided Diebold and Mariano (1995) test of equal predictive ability at the $10 \%, 5 \%, 1 \%$ level. The variance is estimated using Bartlett kernel weights with bandwidth equal to the forecast horizon $h$. The evaluation sample size is $P=72$ quarters. The columns $h=4^{*}$ and $h=8^{*}$ show the results for the one- and two-year-ahead, year-on-year predictions, respectively.

\section{C.4 Hierarchical BVAR}

Tables C.9 to C.12 show results for a BVAR (GLP-BVAR) estimated following Giannone et al. (2015). ${ }^{22}$ The BVAR is estimated using a Minnesota prior with additional "single-unit-root" and a "sum-of-cofficient" prior, where the hyperparameters that control the tightness of the prior are treated as parameters instead of being fixed a priori. The hierarchical BVAR of Giannone et al. (2015) helps to rule out some alternative explanations of why the SPF information helps to improve the forecasting performance. First, the "dummy-initial-observation" and the "sumof-coefficient" prior, implemented via dummy observations addresses the potential problem

\footnotetext{
${ }^{22}$ The BVAR has constant volatilities and is estimated in levels (log-levels respectively). The number of lags is equal to 4 , which is the standard in the literature for quarterly data, and results are robust to different lag length.
} 
of fitting the low-frequency variation in the data via the deterministic component of the VAR (see Giannone et al. (2019) for more details). Second, treating the hyperparameter that controls the tightness of the prior as an additional parameter serves as a robustness check against our hyperparameter choices in the previously discussed models.

Results are qualitatively the same although the improvements for GDP are statistically significant at less horizons whereas the improvements for HICP are larger and more often significant. However, Tables C.13 and C.14 show results when using the multi-horizon forecast comparison test of Quaedvlieg (2019) and results are very similar to the baseline model, including for GDP. Importantly, the bias upward bias in GDP remains as shown in Table C.15.

Table C.9: Entropic tilting versus GLP-BVAR: RMSFE

\begin{tabular}{|c|c|c|c|c|c|c|c|c|c|c|}
\hline Forecast horizon & $h=1$ & $h=2$ & $h=3$ & $h=4$ & $h=5$ & $h=6$ & $h=7$ & $h=8$ & $h=4 *$ & $h=8 *$ \\
\hline & \multicolumn{10}{|c|}{ Panel A. BVAR } \\
\hline GDP & 0.51 & 0.55 & 0.59 & 0.60 & 0.62 & 0.62 & 0.62 & 0.62 & 1.58 & 2.11 \\
\hline $\mathrm{HICP}$ & 0.26 & 0.29 & 0.31 & 0.32 & 0.32 & 0.33 & 0.33 & 0.33 & 1.00 & 1.15 \\
\hline Unemployment & 0.26 & 0.39 & 0.54 & 0.69 & 0.84 & 0.99 & 1.14 & 1.28 & $N / A$ & $N / A$ \\
\hline \multirow[t]{2}{*}{ Euribor } & 0.38 & 0.71 & 1.00 & 1.24 & 1.43 & 1.57 & 1.69 & 1.79 & $N / A$ & $N / A$ \\
\hline & \multicolumn{10}{|c|}{ Panel B. Tilting GDP } \\
\hline GDP & $0.84^{* * *}$ & 0.92 & 0.93 & 0.93 & 0.94 & 0.93 & 0.93 & 0.97 & $0.87^{*}$ & 0.91 \\
\hline $\mathrm{HICP}$ & 1.00 & $0.99^{* * *}$ & 0.99 & 1.00 & 1.00 & 1.00 & 1.00 & 1.00 & 0.99 & 1.01 \\
\hline Unemployment & 0.98 & 0.97 & 0.97 & 0.96 & 0.96 & 0.96 & 0.96 & 0.96 & $N / A$ & $N / A$ \\
\hline \multirow[t]{2}{*}{ Euribor } & 0.97 & 0.94 & $0.93^{*}$ & $0.94^{*}$ & 0.97 & 1.01 & 1.05 & 1.07 & $N / A$ & $N / A$ \\
\hline & \multicolumn{10}{|c|}{ Panel C. Tilting HICP } \\
\hline GDP & $0.97^{*}$ & $0.97^{* *}$ & $0.97^{* * *}$ & 0.98 & 0.99 & 1.00 & 0.99 & 1.00 & $0.96^{* *}$ & 0.99 \\
\hline HICP & $0.89^{* * *}$ & $0.92^{*}$ & $0.87^{* *}$ & $0.86^{* *}$ & $0.87^{* *}$ & $0.87^{* *}$ & $0.87^{* *}$ & $0.87^{* *}$ & $0.84^{* *}$ & $0.82^{* *}$ \\
\hline Unemployment & 1.00 & 1.00 & 1.00 & 1.01 & 1.01 & 1.02 & 1.03 & $1.03^{*}$ & $N / A$ & $N / A$ \\
\hline \multirow[t]{2}{*}{ Euribor } & 0.98 & $0.96^{*}$ & $0.94^{*}$ & $0.92^{*}$ & $0.92^{*}$ & $0.92^{* *}$ & $0.92^{* *}$ & $0.93^{* * *}$ & $N / A$ & $N / A$ \\
\hline & \multicolumn{10}{|c|}{ Panel D. Tilting Unemployment } \\
\hline GDP & 0.97 & 1.05 & 1.07 & 1.01 & 0.99 & 1.01 & 1.00 & $1.06^{* * *}$ & 1.05 & 0.99 \\
\hline HICP & 1.00 & 1.00 & 0.98 & 0.97 & 0.99 & 0.98 & 0.99 & 0.98 & 0.98 & 0.98 \\
\hline Unemployment & 0.99 & 1.00 & 1.02 & 1.04 & 1.05 & 1.03 & 1.01 & 1.00 & $N / A$ & $N / A$ \\
\hline \multirow[t]{2}{*}{ Euribor } & 0.97 & 0.98 & 1.02 & 1.08 & 1.13 & 1.15 & 1.16 & 1.14 & $N / A$ & $N / A$ \\
\hline & \multicolumn{10}{|c|}{ Panel E. Tilting GDP, HICP and Unemployment } \\
\hline GDP & $0.86^{* *}$ & 0.92 & 0.92 & 0.93 & 0.93 & 0.94 & 0.95 & 1.02 & $0.87^{*}$ & 0.91 \\
\hline $\mathrm{HICP}$ & $0.91^{* * *}$ & $0.92^{*}$ & $0.88^{* *}$ & $0.87^{* *}$ & $0.88^{* *}$ & $0.86^{* *}$ & $0.86^{* *}$ & $0.89^{* *}$ & $0.84^{* *}$ & $0.82^{* *}$ \\
\hline Unemployment & 0.99 & 1.00 & 1.02 & 1.04 & 1.03 & 1.02 & 1.01 & 1.00 & $N / A$ & $N / A$ \\
\hline Euribor & 0.98 & $0.90^{* *}$ & $0.89^{* *}$ & $0.91^{*}$ & 0.95 & 0.99 & 1.01 & 1.01 & $N / A$ & $N / A$ \\
\hline
\end{tabular}

Note: Panel A displays the raw GLP-BVAR RMSFE. Panels B to E show the ratios of the RMSFE of the entropic tilted GLP-BVAR to the raw GLP-BVAR. Numbers in bold imply that the tilted GLP-BVAR improves over the raw GLP-BVAR. Asterisks ${ }^{*},{ }^{* *}$ and ${ }^{* * *}$ imply statistical significance of a two-sided Diebold and Mariano (1995) test of equal predictive ability at the $10 \%, 5 \%, 1 \%$ level. The variance is estimated using Bartlett kernel weights with bandwidth equal to the forecast horizon $h$. The evaluation sample size is $P=72$ quarters. The columns $h=4^{*}$ and $h=8^{*}$ show the results for the one- and two-year-ahead, year-on-year predictions, respectively. 
Table C.10: Entropic tilting versus GLP-BVAR: CRPS

\begin{tabular}{|c|c|c|c|c|c|c|c|c|c|c|}
\hline \multirow[t]{3}{*}{ Forecast horizon } & $h=1$ & $h=2$ & $h=3$ & $h=4$ & $h=5$ & $h=6$ & $h=7$ & $h=8$ & $h=4 *$ & $h=8 *$ \\
\hline & \multicolumn{10}{|c|}{ Panel A. BVAR } \\
\hline & 0.32 & 0.33 & 0.34 & 0.36 & 0.36 & 0.37 & 0.38 & 0.37 & 0.88 & 1.22 \\
\hline HICP & 0.15 & 0.16 & 0.17 & 0.18 & 0.18 & 0.18 & 0.19 & 0.18 & 0.57 & 0.65 \\
\hline Unemployment & 0.16 & 0.22 & 0.30 & 0.39 & 0.49 & 0.58 & 0.67 & 0.76 & $N / A$ & $N / A$ \\
\hline \multirow[t]{2}{*}{ Euribor } & 0.19 & 0.37 & 0.53 & 0.67 & 0.79 & 0.89 & 0.97 & 1.05 & $N / A$ & $N / A$ \\
\hline & \multicolumn{10}{|c|}{ Panel B. Tilting GDP } \\
\hline GDP & $0.84^{* *}$ & 0.89 & 0.91 & 0.91 & 0.91 & 0.91 & 0.90 & 0.97 & 0.80 & 0.86 \\
\hline $\mathrm{HICP}$ & 1.00 & $0.99^{* *}$ & 1.00 & 1.01 & 1.01 & 1.01 & 1.02 & 1.01 & 1.00 & 1.02 \\
\hline Unemployment & 0.99 & 0.97 & 0.96 & 0.95 & 0.95 & 0.95 & 0.95 & 0.96 & $N / A$ & $N / A$ \\
\hline \multirow[t]{2}{*}{ Euribor } & 0.98 & $0.96^{*}$ & $0.95^{* *}$ & $0.95^{* *}$ & 0.98 & 1.00 & 1.03 & 1.04 & $N / A$ & $N / A$ \\
\hline & \multicolumn{10}{|c|}{ Panel C. Tilting HICP } \\
\hline GDP & $0.98^{* *}$ & $0.98^{* *}$ & $0.98^{* * *}$ & 0.99 & 0.99 & 1.00 & 0.99 & 1.00 & $0.96^{* *}$ & 0.99 \\
\hline HICP & $0.89^{* * *}$ & $0.93^{*}$ & $0.88^{* *}$ & $0.86^{* *}$ & $0.88^{* *}$ & $0.88^{* *}$ & $0.88^{* *}$ & $0.89^{* * *}$ & $0.86^{* *}$ & $0.86^{* *}$ \\
\hline Unemployment & 1.00 & 1.00 & 1.00 & 1.01 & 1.02 & $1.03^{*}$ & $1.03^{*}$ & $1.04^{*}$ & $N / A$ & $N / A$ \\
\hline \multirow[t]{2}{*}{ Euribor } & 0.98 & $0.97^{*}$ & $0.96^{*}$ & $0.94^{*}$ & $0.93^{* *}$ & $0.93^{* *}$ & $0.93^{* * *}$ & $0.93^{* * *}$ & $N / A$ & $N / A$ \\
\hline & \multicolumn{10}{|c|}{ Panel D. Tilting Unemployment } \\
\hline GDP & 1.02 & $1.10^{*}$ & 1.08 & 1.01 & 0.98 & 1.01 & 1.00 & $1.05^{* * *}$ & 1.08 & 0.98 \\
\hline $\mathrm{HICP}$ & 1.00 & 1.00 & 0.98 & 0.97 & 0.98 & 0.98 & 1.00 & 1.00 & 0.98 & 0.99 \\
\hline Unemployment & 0.99 & 1.01 & 1.03 & 1.03 & 1.04 & 1.03 & 1.02 & 1.02 & $N / A$ & $N / A$ \\
\hline \multirow[t]{2}{*}{ Euribor } & 0.98 & 0.97 & 1.02 & 1.08 & 1.12 & 1.13 & 1.12 & 1.10 & $N / A$ & $N / A$ \\
\hline & \multicolumn{10}{|c|}{ Panel E. Tilting GDP, HICP and Unemployment } \\
\hline GDP & $0.87^{*}$ & 0.91 & 0.90 & 0.92 & 0.91 & 0.92 & 0.93 & 1.03 & 0.80 & 0.86 \\
\hline $\mathrm{HICP}$ & $0.92^{* * *}$ & 0.94 & $0.89^{* *}$ & $0.89^{* *}$ & $0.90^{* *}$ & $0.87^{* *}$ & $0.89^{* * *}$ & $0.91^{* *}$ & $0.87^{* *}$ & $0.87^{* *}$ \\
\hline Unemployment & 0.99 & 1.04 & 1.07 & 1.08 & 1.06 & 1.04 & 1.02 & 1.02 & $N / A$ & $N / A$ \\
\hline Euribor & 1.00 & 0.93 & $0.93^{*}$ & 0.95 & 0.97 & 0.99 & 1.00 & 0.99 & $N / A$ & $N / A$ \\
\hline
\end{tabular}

Note: Panel A displays the raw GLP-BVAR CRPS. Panels B to E show the ratios of the CRPS of the entropic tilted GLP-BVAR to the raw GLP-BVAR. Numbers in bold imply that the tilted GLP-BVAR improves over the raw GLP-BVAR. Asterisks *, ** and ${ }^{* * *}$ imply statistical significance of a two-sided Diebold and Mariano (1995) test of equal predictive ability at the $10 \%$, $5 \%, 1 \%$ level. The variance is estimated using Bartlett kernel weights with bandwidth equal to the forecast horizon $h$. The evaluation sample size is $P=72$ quarters. The columns $h=4^{*}$ and $h=8^{*}$ show the results for the one- and two-year-ahead, year-on-year predictions, respectively. 
Table C.11: Soft Conditioning versus GLP-BVAR: RMSFE

\begin{tabular}{|c|c|c|c|c|c|c|c|c|c|c|}
\hline \multicolumn{2}{|c|}{ Forecast horizon $h=1$} & $h=2$ & $h=3$ & $h=4$ & $h=5$ & $h=6$ & $h=7$ & $h=8$ & $h=4 *$ & $h=8 *$ \\
\hline & \multicolumn{10}{|c|}{ Panel A. BVAR } \\
\hline GDP & 0.51 & 0.55 & 0.59 & 0.60 & 0.62 & 0.62 & 0.62 & 0.62 & 1.58 & 2.11 \\
\hline HICP & 0.26 & 0.29 & 0.31 & 0.32 & 0.32 & 0.33 & 0.33 & 0.33 & 1.00 & 1.15 \\
\hline Unemployment & 0.26 & 0.39 & 0.54 & 0.69 & 0.84 & 0.99 & 1.14 & 1.28 & $N / A$ & $N / A$ \\
\hline \multirow[t]{2}{*}{ Euribor } & 0.38 & 0.71 & 1.00 & 1.24 & 1.43 & 1.57 & 1.69 & 1.79 & $N / A$ & $N / A$ \\
\hline & \multicolumn{10}{|c|}{ Panel B. Soft Conditioning GDP } \\
\hline GDP & $0.86^{* *}$ & 0.91 & 0.92 & 0.91 & 0.92 & 0.92 & 0.92 & 0.97 & $0.87^{*}$ & 0.89 \\
\hline HICP & 1.00 & $0.99^{* *}$ & 0.99 & 0.99 & 0.99 & 1.00 & 1.00 & 0.99 & 0.99 & 1.00 \\
\hline Unemployment & 0.99 & 0.98 & 0.98 & 0.97 & 0.97 & 0.97 & 0.97 & 0.97 & $N / A$ & $N / A$ \\
\hline \multirow[t]{2}{*}{ Euribor } & 0.97 & 0.95 & 0.94 & $0.94^{*}$ & $0.97^{*}$ & 1.00 & 1.02 & 1.04 & $N / A$ & $N / A$ \\
\hline & \multicolumn{10}{|c|}{ Panel C. Soft Conditioning HICP } \\
\hline GDP & $0.98^{*}$ & $0.99^{* *}$ & $0.99^{* *}$ & 0.99 & 1.00 & 1.00 & 1.00 & 1.00 & $0.98^{* *}$ & 1.00 \\
\hline HICP & $0.95^{* * *}$ & $0.95^{* *}$ & $0.92^{* *}$ & $0.90^{* *}$ & $0.91^{* *}$ & $0.90^{* *}$ & $0.89^{* *}$ & $0.90^{* *}$ & $0.90^{* * *}$ & $0.86^{* *}$ \\
\hline Unemployment & 1.00 & 1.00 & 1.00 & 1.00 & 1.01 & $1.01^{*}$ & $1.01^{* *}$ & $1.02^{* *}$ & $N / A$ & $N / A$ \\
\hline \multirow[t]{2}{*}{ Euribor } & $0.98^{*}$ & $0.97^{*}$ & $0.96^{*}$ & $0.94^{*}$ & $0.94^{* *}$ & $0.95^{* *}$ & $0.95^{* *}$ & $0.95^{* * *}$ & $N / A$ & $N / A$ \\
\hline & \multicolumn{10}{|c|}{ Panel D. Soft Conditioning Unemployment } \\
\hline GDP & 1.00 & 1.01 & 1.02 & 1.01 & 0.99 & 0.99 & 0.99 & 0.99 & 1.02 & 0.99 \\
\hline HICP & 0.99 & 0.99 & 0.98 & 0.98 & 0.98 & 0.97 & 0.97 & 0.97 & 0.98 & 0.97 \\
\hline Unemployment & 1.00 & 0.99 & 0.99 & 0.99 & 1.00 & 1.00 & 0.99 & 0.98 & $N / A$ & $N / A$ \\
\hline \multirow[t]{2}{*}{ Euribor } & 1.00 & 1.00 & 1.00 & 1.01 & 1.03 & 1.05 & 1.06 & 1.06 & $N / A$ & $N / A$ \\
\hline & \multicolumn{10}{|c|}{ Panel E. Soft Conditioning GDP, HICP and Unemployment } \\
\hline GDP & $0.86^{* *}$ & 0.91 & 0.92 & 0.91 & 0.92 & 0.92 & 0.92 & 0.97 & $0.87^{*}$ & 0.89 \\
\hline HICP & $0.95^{* * *}$ & $0.95^{* *}$ & $0.92^{* *}$ & $0.90^{* *}$ & $0.91^{* *}$ & $0.90^{*}$ & $0.90^{*}$ & $0.90^{*}$ & $0.90^{* * *}$ & $0.87^{*}$ \\
\hline Unemployment & 0.99 & 0.98 & 0.98 & 0.98 & 0.98 & 0.99 & 0.99 & 0.98 & $N / A$ & $N / A$ \\
\hline Euribor & 0.96 & $0.93^{*}$ & $0.91^{* *}$ & $0.91^{* *}$ & $0.93^{* * *}$ & $0.96^{* * *}$ & 0.98 & 1.00 & $N / A$ & $N / A$ \\
\hline
\end{tabular}

Note: Panel A displays the raw GLP-BVAR RMSFE. Panels B to E show the ratios of the RMSFE of the soft conditioned GLP-BVAR to the raw GLP-BVAR. Numbers in bold imply that the soft conditioned GLP-BVAR improves over the raw GLP-BVAR. Asterisks *, ${ }^{* *}$ and ${ }^{* * *}$ imply statistical significance of a two-sided Diebold and Mariano (1995) test of equal predictive ability at the $10 \%, 5 \%, 1 \%$ level. The variance is estimated using Bartlett kernel weights with bandwidth equal to the forecast horizon $h$. The evaluation sample size is $P=72$ quarters. The columns $h=4^{*}$ and $h=8^{*}$ show the results for the one- and two-year-ahead, year-on-year predictions, respectively. 
Table C.12: Soft Conditioning versus GLP-BVAR: CRPS

\begin{tabular}{|c|c|c|c|c|c|c|c|c|c|c|}
\hline \multicolumn{2}{|c|}{ Forecast horizon $h=1$} & $h=2$ & $h=3$ & $h=4$ & $h=5$ & $h=6$ & $h=7$ & $h=8$ & $h=4 *$ & $h=8 *$ \\
\hline & \multicolumn{10}{|c|}{ Panel A. BVAR } \\
\hline GDP & 0.32 & 0.33 & 0.34 & 0.36 & 0.36 & 0.37 & 0.38 & 0.37 & 0.88 & 1.22 \\
\hline НICP & 0.15 & 0.16 & 0.17 & 0.18 & 0.18 & 0.18 & 0.19 & 0.18 & 0.57 & 0.65 \\
\hline Unemployment & 0.16 & 0.22 & 0.30 & 0.39 & 0.49 & 0.58 & 0.67 & 0.76 & $N / A$ & $N / A$ \\
\hline \multirow[t]{2}{*}{ Euribor } & 0.19 & 0.37 & 0.53 & 0.67 & 0.79 & 0.89 & 0.97 & 1.05 & $N / A$ & $N / A$ \\
\hline & \multicolumn{10}{|c|}{ Panel B. Soft Conditioning GDP } \\
\hline GDP & $0.85^{* *}$ & $0.88^{*}$ & 0.89 & 0.90 & 0.90 & 0.90 & 0.90 & 0.97 & 0.80 & 0.84 \\
\hline НICP & 1.00 & 1.00 & 1.00 & 1.00 & 1.00 & 1.01 & 1.01 & 1.00 & 1.00 & 1.01 \\
\hline Unemployment & 0.99 & 0.99 & 0.98 & 0.97 & 0.97 & 0.97 & 0.97 & 0.98 & $N / A$ & $N / A$ \\
\hline \multirow[t]{2}{*}{ Euribor } & 0.98 & $0.97^{*}$ & $0.96^{* *}$ & $0.96^{* *}$ & 0.98 & 1.00 & 1.01 & 1.02 & $N / A$ & $N / A$ \\
\hline & \multicolumn{10}{|c|}{ Panel C. Soft Conditioning HICP } \\
\hline GDP & $0.99^{* *}$ & $0.99^{* * *}$ & $0.99^{* * *}$ & $0.99^{*}$ & 1.00 & 1.00 & 1.00 & 1.00 & $0.98^{* *}$ & 1.00 \\
\hline HICP & $0.95^{* * *}$ & $0.96^{*}$ & $0.93^{* *}$ & $0.92^{* *}$ & $0.92^{* *}$ & $0.92^{* *}$ & $0.91^{* *}$ & $0.92^{* *}$ & $0.94^{* *}$ & $0.91^{* *}$ \\
\hline Unemployment & 1.00 & 1.00 & 1.00 & 1.01 & $1.01^{* *}$ & $1.02^{* *}$ & $1.02^{* *}$ & $1.02^{* *}$ & $N / A$ & $N / A$ \\
\hline \multirow[t]{2}{*}{ Euribor } & $0.99^{*}$ & $0.98^{* *}$ & $0.97^{* *}$ & $0.96^{* *}$ & $0.96^{* *}$ & $0.95^{* * *}$ & $0.95^{* * *}$ & $0.96^{* * *}$ & $N / A$ & $N / A$ \\
\hline & \multicolumn{10}{|c|}{ Panel D. Soft Conditioning Unemployment } \\
\hline GDP & 1.00 & 1.01 & 1.02 & 1.00 & 0.99 & 0.99 & $0.98^{* * *}$ & $0.99^{* *}$ & 1.03 & 0.99 \\
\hline HICP & 0.99 & 0.99 & 0.99 & 0.98 & 0.98 & 0.98 & 0.99 & 0.99 & 0.98 & 0.98 \\
\hline Unemployment & 1.00 & 1.00 & 1.00 & 1.00 & 1.01 & 1.02 & 1.02 & 1.01 & $N / A$ & $N / A$ \\
\hline \multirow[t]{2}{*}{ Euribor } & 1.00 & 0.99 & 0.99 & 1.00 & 1.02 & 1.03 & 1.03 & 1.03 & $N / A$ & $N / A$ \\
\hline & \multicolumn{10}{|c|}{ Panel E. Soft Conditioning GDP, HICP and Unemployment } \\
\hline GDP & $0.85^{* *}$ & $0.88^{*}$ & 0.89 & 0.90 & $0.90^{\circ}$ & 0.90 & 0.90 & 0.97 & 0.80 & 0.83 \\
\hline HICP & $0.95^{* *}$ & $0.96^{*}$ & $0.93^{* *}$ & $0.92^{* *}$ & $0.93^{*}$ & $0.93^{* *}$ & 0.93 & $0.93^{*}$ & $0.93^{*}$ & 0.93 \\
\hline Unemployment & 1.00 & 1.00 & 1.00 & 1.00 & 1.01 & 1.02 & 1.02 & 1.03 & $N / A$ & $N / A$ \\
\hline Euribor & 0.97 & $0.95^{* *}$ & $0.93^{* * *}$ & $0.93^{* * *}$ & $0.95^{* *}$ & 0.96 & 0.98 & 0.98 & $N / A$ & $N / A$ \\
\hline
\end{tabular}

Note: Panel A displays the raw GLP-BVAR CRPS. Panels B to E show the ratios of the CRPS of the soft conditioned GLP-BVAR to the raw GLP-BVAR. Numbers in bold imply that the soft conditioned GLP-BVAR improves over the raw GLP-BVAR. Asterisks *, ${ }^{* *}$ and ${ }^{* * *}$ imply statistical significance of a two-sided Diebold and Mariano (1995) test of equal predictive ability at the $10 \%, 5 \%, 1 \%$ level. The variance is estimated using Bartlett kernel weights with bandwidth equal to the forecast horizon $h$. The evaluation sample size is $P=72$ quarters. The columns $h=4^{*}$ and $h=8^{*}$ show the results for the one- and two-year-ahead, year-on-year predictions, respectively. 
Table C.13: Entropic tilting versus GLP-BVAR: Multi-Horizon Test

\begin{tabular}{|c|c|c|c|c|c|c|c|c|}
\hline & \multicolumn{8}{|c|}{ Panel A. Tilting GDP } \\
\hline & \multicolumn{4}{|c|}{ RMSFE } & \multicolumn{4}{|c|}{ CRPS } \\
\hline & GDP & $\mathrm{HICP}$ & UMP & INTR & GDP & $\mathrm{HICP}$ & UMP & INTR \\
\hline$h=1-4$ & $1.82^{* *}$ & 0.67 & $1.35^{* *}$ & $1.44^{* * *}$ & $2.35^{* *}$ & 0.03 & $1.17^{* *}$ & $1.72^{* * *}$ \\
\hline$h=1-8$ & $1.63^{*}$ & 0.09 & 1.11 & -0.38 & $2.14^{* *}$ & -0.30 & 1.06 & 0.13 \\
\hline
\end{tabular}

Panel B. Tilting HICP

\begin{tabular}{|c|c|c|c|c|c|c|c|c|}
\hline & \multicolumn{4}{|c|}{ RMSFE } & \multicolumn{4}{|c|}{ CRPS } \\
\hline & GDP & HICP & UMP & INTR & GDP & HICP & UMP & INTR \\
\hline & $2.33^{* * *}$ & $2.29^{* * *}$ & -0.23 & $1.83^{* * *}$ & $2.75^{* * *}$ & $2.34^{* * *}$ & -0.37 & $1.90^{* *}$ \\
\hline & $2.03^{* *}$ & $2.44^{* *}$ & -1.26 & $2.21^{* *}$ & $2.56^{* *}$ & $2.54^{* *}$ & -1.42 & $2.32^{* *}$ \\
\hline
\end{tabular}

Panel C. Tilting Unemployment

\begin{tabular}{|c|c|c|c|c|c|c|c|c|}
\hline & \multicolumn{4}{|c|}{ MVIV L } & \multicolumn{4}{|c|}{ CN } \\
\hline & GDP & НICP & UMP & INTR & GDP & HICP & UMP & INTR \\
\hline & 0.76 & 0.38 & -0.59 & -0.61 & -1.46 & 0.34 & -0.31 & -0.48 \\
\hline & -1.04 & 0.30 & -0.38 & -1.25 & -1.70 & 0.27 & -0.32 & -1.09 \\
\hline
\end{tabular}

Panel D. Tilting Jointly

\begin{tabular}{|c|c|c|c|c|c|c|c|c|}
\hline & \multicolumn{4}{|c|}{ RMSFE } & \multicolumn{4}{|c|}{ CRPS } \\
\hline & GDP & HICP & UMP & INTR & GDP & HICP & UMP & INTR \\
\hline 1 & $1.84^{* *}$ & $2.17^{* * *}$ & -0.50 & $1.58^{* *}$ & $2.17^{* *}$ & $2.08^{* *}$ & -0.77 & $1.12^{* *}$ \\
\hline-1 & $1.42^{*}$ & $2.36^{* *}$ & -0.28 & 0.55 & $1.79^{*}$ & $2.31^{* *}$ & -0.52 & 0.52 \\
\hline
\end{tabular}

Note: The table shows the results of the "multi-horizon forecast comparison test" by Quaedvlieg (2019). Positive (bold) numbers imply that the tilted GLP-BVAR improved over the raw GLP-BVAR. Asterisks *,** and ${ }^{* * *}$ imply statistical significance of the one-sided multi-horizon test at the $10 \%, 5 \%, 1 \%$ level. The evaluation sample size is $P=72$ quarters. The rows $\mathrm{h}=1-4$ and $h=1-8$ show the results when applying the multi-horizon test to forecast horizons $h=1$ to 4 and $\mathrm{h}=1$ to 8 respectively. 
Table C.14: Soft Conditioning versus GLP-BVAR: Multi-Horizon Test

\begin{tabular}{|c|c|c|c|c|c|c|c|c|}
\hline & \multicolumn{8}{|c|}{ Panel A. Tilting GDP } \\
\hline & \multicolumn{4}{|c|}{ RMSFE } & \multicolumn{4}{|c|}{ CRPS } \\
\hline & GDP & $\mathrm{HICP}$ & UMP & INTR & GDP & $\mathrm{HICP}$ & UMP & INTR \\
\hline$h=1-4$ & $2.09^{* *}$ & 0.75 & $1.41^{* *}$ & $1.38^{* * *}$ & $2.87^{* * *}$ & 0.00 & $1.02^{*}$ & $1.69^{* *}$ \\
\hline$h=1-8$ & $1.97^{* *}$ & 0.36 & 1.19 & 0.21 & $2.64^{* *}$ & -0.15 & 0.84 & 0.46 \\
\hline
\end{tabular}

Panel B. Tilting HICP

\begin{tabular}{|c|c|c|c|c|c|c|c|c|}
\hline & \multicolumn{4}{|c|}{ RMSFE } & \multicolumn{4}{|c|}{ CRPS } \\
\hline & GDP & HICP & UMP & INTR & GDP & HICP & UMP & INTR \\
\hline $1-4$ & $2.58^{* * *}$ & $2.36^{* * *}$ & -0.62 & $1.81^{* * *}$ & $3.28^{* * *}$ & $2.14^{* * *}$ & -0.98 & $2.25^{* * *}$ \\
\hline$h=1$ & $1.82^{* *}$ & $2.26^{* * *}$ & -1.68 & $2.27^{* *}$ & $2.94^{* * *}$ & $2.29^{* * *}$ & -2.01 & $2.68^{* * *}$ \\
\hline
\end{tabular}

Panel C. Tilting Unemployment

\begin{tabular}{|c|c|c|c|c|c|c|c|c|}
\hline & \multicolumn{4}{|c|}{ Tूर } & \\
\hline & GDP & HICP & UMP & INTR & GDP & HICP & UMP & INTR \\
\hline & -0.91 & $0.79^{*}$ & 0.50 & -0.34 & -0.80 & $0.69^{*}$ & -0.05 & 0.16 \\
\hline & 0.05 & 0.69 & 0.37 & -1.14 & 0.12 & 0.54 & -0.35 & -0.70 \\
\hline
\end{tabular}

Panel D. Tilting Jointly

\begin{tabular}{|c|c|c|c|c|c|c|c|c|}
\hline & \multicolumn{4}{|c|}{ RMSFE } & \multicolumn{4}{|c|}{ CRPS } \\
\hline & GDP & HICP & UMP & INTR & GDP & HICP & UMP & INTR \\
\hline & $2.12^{* *}$ & $2.30^{* * *}$ & $0.96^{*}$ & $1.81^{* * *}$ & $2.87^{* * *}$ & $1.97^{* * *}$ & 0.09 & $2.58^{* * *}$ \\
\hline-1 & $2.04^{* *}$ & $2.12^{* * *}$ & 0.53 & $2.07^{* *}$ & $2.68^{* *}$ & $1.93^{* *}$ & -0.34 & $1.78^{*}$ \\
\hline
\end{tabular}

Note: The table shows the results of the "multi-horizon forecast comparison test" by Quaedvlieg (2019). Positive (bold) numbers imply that the tilted GLP-BVAR improved over the raw GLP-BVAR. Asterisks *,** and ${ }^{* * *}$ imply statistical significance of the one-sided multi-horizon test at the $10 \%, 5 \%, 1 \%$ level. The evaluation sample size is $P=72$ quarters. The rows $\mathrm{h}=1-4$ and $h=1-8$ show the results when applying the multi-horizon test to forecast horizons $h=1$ to 4 and $\mathrm{h}=1$ to 8 respectively. 
Table C.15: GLP-BVAR Forecast bias - before and after the Great Recession

\begin{tabular}{|c|c|c|c|c|c|c|c|c|c|c|}
\hline \multirow[t]{2}{*}{ Forecast horizon } & $h=1$ & $h=2$ & $h=3$ & $h=4$ & $h=5$ & $h=6$ & $h=7$ & $h=8$ & $h=4^{*}$ & $h=8^{*}$ \\
\hline & \multicolumn{10}{|c|}{ Panel A. BVAR - Before Great Recession } \\
\hline GDP & -0.01 & -0.01 & -0.03 & -0.06 & -0.07 & -0.09 & -0.11 & -0.11 & -0.21 & -0.46 \\
\hline $\mathrm{HICP}$ & $0.08^{* *}$ & $0.10^{* *}$ & $0.11^{*}$ & $0.11^{*}$ & $0.12^{*}$ & $0.12^{*}$ & $0.12^{*}$ & 0.12 & 0.34 & 0.37 \\
\hline Unemployment & -0.04 & -0.05 & -0.08 & -0.11 & -0.13 & -0.13 & -0.11 & -0.09 & $N / A$ & $N / A$ \\
\hline \multirow[t]{2}{*}{ Euribor } & 0.07 & 0.19 & 0.33 & 0.46 & 0.55 & 0.63 & 0.67 & 0.69 & $N / A$ & $N / A$ \\
\hline & \multicolumn{10}{|c|}{ Panel C. Tilting GDP One-Year and Two-Years-Ahead - Before Great Recession } \\
\hline GDP & -0.05 & -0.06 & -0.09 & -0.13 & -0.14 & -0.15 & -0.15 & -0.13 & -0.37 & -0.72 \\
\hline HICP & $0.08^{* *}$ & $0.10^{* *}$ & $0.11^{*}$ & $0.11^{*}$ & $0.11^{*}$ & $0.11^{*}$ & $0.12^{*}$ & 0.11 & 0.34 & 0.36 \\
\hline Unemployment & -0.03 & -0.04 & -0.06 & -0.07 & -0.07 & -0.05 & -0.02 & 0.03 & $N / A$ & $N / A$ \\
\hline Euribor & 0.09 & 0.21 & 0.34 & 0.44 & 0.49 & 0.51 & 0.49 & 0.45 & $N / A$ & $N / A$ \\
\hline \multicolumn{11}{|c|}{ Panel D. BVAR - After Great Recession } \\
\hline GDP & $-0.24^{* * *}$ & $-0.32^{* * *}$ & $-0.39^{* * *}$ & $-0.43^{* * *}$ & ${ }^{*}-0.45^{* * *}$ & $-0.45^{* * *}$ & $-0.43^{* * *}$ & $-0.43^{* * *}$ & $-0.86^{* *}$ & $-1.73^{* * *}$ \\
\hline HICP & -0.01 & -0.02 & -0.05 & -0.07 & -0.09 & -0.10 & -0.11 & -0.13 & -0.12 & -0.42 \\
\hline Unemployment & 0.03 & 0.08 & 0.13 & 0.20 & 0.27 & 0.34 & 0.40 & 0.46 & $N / A$ & $N / A$ \\
\hline \multirow[t]{2}{*}{ Euribor } & 0.06 & 0.08 & 0.06 & -0.01 & -0.12 & -0.27 & -0.43 & $-0.59^{*}$ & $N / A$ & $N / A$ \\
\hline & \multicolumn{10}{|c|}{ Panel F. Tilting GDP One-Year and Two-Years-Ahead - After Great Recession } \\
\hline GDP & -0.01 & -0.08 & -0.12 & -0.12 & -0.10 & -0.12 & -0.12 & $-0.32^{* * *}$ & -0.09 & -0.36 \\
\hline HICP & -0.02 & -0.02 & -0.04 & -0.05 & -0.06 & -0.06 & -0.07 & -0.09 & -0.10 & -0.27 \\
\hline Unemployment & 0.02 & 0.05 & 0.09 & 0.12 & 0.15 & 0.17 & 0.19 & 0.22 & $N / A$ & $N / A$ \\
\hline Euribor & 0.06 & 0.08 & 0.08 & 0.05 & 0.02 & -0.02 & -0.06 & -0.13 & $N / A$ & $N / A$ \\
\hline
\end{tabular}

Note: Panels A to F display the means of the forecast errors of the GLP-BVAR and tilted GLP-BVAR, before and after the Great Recession. Asterisks ${ }^{*},{ }^{* *}$ and ${ }^{* * *}$ imply statistical significance of a two-sided $t$-test of unbiasedness at the $10 \%, 5 \%, 1 \%$ level. The variance is estimated using Bartlett kernel weights with bandwidth equal to the forecast horizon $h$. The evaluation sample size is $37-h$ quarters in Panels A to C, and 30 quarters in Panels D to F. The columns labeled $h=4^{*}$ and $h=8^{*}$ show the results for the one-year-ahead and two-year-ahead, year-on-year predictions, respectively. 


\section{C.5 BVAR in Levels}

As a final robustness check, we re-estimated the baseline BVAR with stochastic volatility in levels with the standard Minnesota prior. Tables C.16 to C.19 show the results for entropic tilting and soft conditioning for the baseline BVAR, where real GDP and HICP enter the model in log-levels instead of log-differences. We adopted the most common prior specification in this case, which is to shrink the coefficient of the first own-lag to one.

Table C.16: Entropic tilting versus BVAR in levels: RMSFE

\begin{tabular}{|c|c|c|c|c|c|c|c|c|c|c|}
\hline \multirow{2}{*}{\multicolumn{2}{|c|}{ Forecast horizon $h=1$}} & $h=2$ & $h=3$ & $h=4$ & $h=5$ & $h=6$ & $h=7$ & $h=8$ & $h=4 *$ & $h=8 *$ \\
\hline & & \multicolumn{9}{|c|}{ Panel A. BVAR } \\
\hline GDP & 0.54 & 0.58 & 0.58 & 0.57 & 0.56 & 0.57 & 0.56 & 0.56 & 1.67 & 1.88 \\
\hline HICP & 0.29 & 0.32 & 0.35 & 0.36 & 0.36 & 0.36 & 0.36 & 0.36 & 1.15 & 1.30 \\
\hline Unemployment & 0.27 & 0.42 & 0.60 & 0.79 & 0.98 & 1.17 & 1.35 & 1.53 & $N / A$ & $N / A$ \\
\hline \multirow[t]{2}{*}{ Euribor } & 0.40 & 0.76 & 1.10 & 1.43 & 1.73 & 2.00 & 2.24 & 2.46 & $N / A$ & $N / A$ \\
\hline & \multicolumn{10}{|c|}{ Panel B. Tilting GDP } \\
\hline GDP & $0.82^{* *}$ & $0.87^{* *}$ & $0.93^{* *}$ & 0.99 & 1.02 & 1.03 & 1.04 & 1.03 & $0.82^{*}$ & 1.02 \\
\hline $\mathrm{HICP}$ & 0.99 & 1.00 & 1.00 & 1.00 & 1.01 & 1.02 & 1.03 & 1.03 & 1.00 & 1.03 \\
\hline Unemployment & 0.94 & 0.90 & $0.89^{* *}$ & $0.89^{* *}$ & $0.89^{* *}$ & $0.90^{* *}$ & $0.91^{* *}$ & $0.92^{* *}$ & $N / A$ & $N / A$ \\
\hline \multirow[t]{2}{*}{ Euribor } & 1.01 & 0.99 & 0.97 & 0.97 & 0.97 & 0.97 & 0.97 & 0.97 & $N / A$ & $N / A$ \\
\hline & \multicolumn{10}{|c|}{ Panel C. Tilting HICP } \\
\hline GDP & 0.99 & 0.98 & 0.98 & 0.99 & 1.00 & 1.01 & 1.01 & 1.02 & 0.97 & 1.00 \\
\hline HICP & $0.82^{* *}$ & $0.83^{* *}$ & $0.78^{* *}$ & $0.79^{* *}$ & $0.79^{*}$ & $0.79^{*}$ & $0.79^{*}$ & $0.79^{*}$ & $0.72^{* *}$ & $0.72^{*}$ \\
\hline Unemployment & 0.97 & 0.97 & 0.98 & 0.98 & 0.99 & 0.99 & 1.00 & 1.00 & $N / A$ & $N / A$ \\
\hline \multirow[t]{2}{*}{ Euribor } & 0.99 & 1.00 & 1.01 & 1.01 & 1.01 & 1.00 & 0.99 & 0.98 & $N / A$ & $N / A$ \\
\hline & \multicolumn{10}{|c|}{ Panel D. Tilting Unemployment } \\
\hline GDP & 0.96 & 0.96 & 0.98 & 0.97 & 0.97 & 1.01 & 0.98 & 0.99 & 0.96 & 0.96 \\
\hline HICP & 1.00 & 1.00 & 1.01 & 1.00 & 0.99 & 0.99 & 1.01 & 1.02 & 1.00 & 1.00 \\
\hline Unemployment & 0.96 & 0.93 & 0.91 & 0.90 & $0.89^{*}$ & $0.87^{* * *}$ & $0.85^{* * *}$ & $0.83^{* * *}$ & $N / A$ & $N / A$ \\
\hline \multirow[t]{2}{*}{ Euribor } & 0.99 & 0.98 & 0.99 & 1.01 & 1.02 & 1.01 & 1.01 & 0.99 & $N / A$ & $N / A$ \\
\hline & \multicolumn{10}{|c|}{ Panel E. Tilting GDP, HICP and Unemployment } \\
\hline GDP & $0.85^{*}$ & $0.87^{* *}$ & $0.92^{* *}$ & 1.02 & 1.02 & 1.05 & 1.05 & 1.03 & $0.82^{*}$ & 1.02 \\
\hline $\mathrm{HICP}$ & $0.84^{* *}$ & $0.83^{* *}$ & $0.80^{* *}$ & $0.81^{* *}$ & $0.79^{* *}$ & $0.78^{*}$ & $0.80^{*}$ & 0.81 & $0.73^{* *}$ & $0.72^{*}$ \\
\hline Unemployment & 0.93 & 0.90 & 0.89 & 0.90 & $0.89^{*}$ & $0.87^{* * *}$ & $0.85^{* * *}$ & $0.84^{* * *}$ & $N / A$ & $N / A$ \\
\hline Euribor & 0.99 & 0.99 & 0.97 & 0.98 & 0.98 & 0.96 & 0.95 & 0.93 & $N / A$ & $N / A$ \\
\hline
\end{tabular}

Note: Panel A displays the raw BVAR in levels RMSFE. Panels B to E show the ratios of the RMSFE of the entropic tilted BVAR in levels to the raw BVAR in levels. Numbers in bold imply that the tilted BVAR in levels improves over the raw BVAR in levels. Asterisks *, ${ }^{* *}$ and ${ }^{* * *}$ imply statistical significance of a two-sided Diebold and Mariano (1995) test of equal predictive ability at the $10 \%, 5 \%, 1 \%$ level. The variance is estimated using Bartlett kernel weights with bandwidth equal to the forecast horizon $h$. The evaluation sample size is $P=72$ quarters. The columns $h=4^{*}$ and $h=8^{*}$ show the results for the one- and two-year-ahead, year-on-year predictions, respectively. 
Table C.17: Entropic tilting versus BVAR in levels: CRPS

\begin{tabular}{|c|c|c|c|c|c|c|c|c|c|c|}
\hline \multirow[t]{2}{*}{ Forecast horizon $l$} & $h=1$ & $h=2$ & $h=3$ & $h=4$ & $h=5$ & $h=6$ & $h=7$ & $h=8$ & $h=4 *$ & $h=8 *$ \\
\hline & \multicolumn{10}{|c|}{ Panel A. BVAR } \\
\hline GDP & 0.26 & 0.27 & 0.28 & 0.27 & 0.28 & 0.27 & 0.27 & 0.27 & 0.81 & 1.05 \\
\hline HICP & 0.16 & 0.18 & 0.20 & 0.21 & 0.20 & 0.20 & 0.21 & 0.21 & 0.67 & 0.76 \\
\hline Unemployment & 0.16 & 0.24 & 0.36 & 0.49 & 0.63 & 0.76 & 0.90 & 1.03 & $N / A$ & $N / A$ \\
\hline \multirow[t]{2}{*}{ Euribor } & 0.19 & 0.39 & 0.58 & 0.78 & 0.96 & 1.11 & 1.26 & 1.38 & $N / A$ & $N / A$ \\
\hline & \multicolumn{10}{|c|}{ Panel B. Tilting GDP } \\
\hline GDP & $0.83^{* * *}$ & $0.86^{* * *}$ & $0.90^{* * *}$ & $0.94^{*}$ & 0.97 & 0.97 & 0.97 & 0.99 & $0.83^{* *}$ & 1.00 \\
\hline HICP & 0.99 & 1.00 & 1.01 & 1.01 & 1.02 & 1.03 & 1.04 & 1.04 & 1.01 & $1.06^{*}$ \\
\hline Unemployment & 0.96 & $0.92^{*}$ & $0.90^{* *}$ & $0.89^{* *}$ & $0.89^{* *}$ & $0.90^{* * *}$ & $0.90^{* * *}$ & $0.91^{* * *}$ & $N / A$ & $N / A$ \\
\hline \multirow[t]{2}{*}{ Euribor } & 1.02 & 1.02 & 1.01 & 1.01 & 1.01 & 1.01 & 1.00 & 1.00 & $N / A$ & $N / A$ \\
\hline & \multicolumn{10}{|c|}{ Panel C. Tilting HICP } \\
\hline GDP & 0.99 & 0.99 & 0.98 & 0.98 & 1.00 & 1.01 & 1.02 & 1.02 & 0.99 & 1.01 \\
\hline HICP & $0.83^{* * *}$ & $0.83^{* *}$ & $0.79^{* *}$ & $0.79^{* *}$ & $0.80^{*}$ & $0.79^{*}$ & $0.79^{*}$ & $0.79^{*}$ & $0.74^{* * *}$ & $0.74^{*}$ \\
\hline Unemployment & 0.98 & 0.98 & 1.00 & 1.01 & 1.01 & 1.01 & 1.01 & 1.01 & $N / A$ & $N / A$ \\
\hline \multirow[t]{2}{*}{ Euribor } & 1.01 & 1.02 & 1.02 & 1.02 & 1.02 & 1.02 & 1.01 & 1.01 & $N / A$ & $N / A$ \\
\hline & \multicolumn{10}{|c|}{ Panel D. Tilting Unemployment } \\
\hline GDP & 0.98 & 0.97 & 0.97 & 0.95 & 0.96 & 0.99 & 0.96 & 0.98 & 0.95 & 0.93 \\
\hline $\mathrm{HICP}$ & 1.00 & 0.99 & 1.00 & 0.98 & 0.98 & 0.99 & 1.01 & 1.02 & 0.99 & 1.00 \\
\hline Unemployment & 0.96 & 0.95 & 0.91 & 0.88 & $0.85^{*}$ & $0.82^{*}$ & $0.80^{* *}$ & $0.78^{* *}$ & $N / A$ & $N / A$ \\
\hline \multirow[t]{2}{*}{ Euribor } & 1.00 & 0.99 & 1.00 & 1.02 & 1.02 & 1.02 & 1.02 & 1.01 & $N / A$ & $N / A$ \\
\hline & \multicolumn{10}{|c|}{ Panel E. Tilting GDP, HICP and Unemployment } \\
\hline GDP & $0.87^{* * *}$ & $0.87^{* * *}$ & $0.91^{* * *}$ & 0.96 & 0.96 & 1.00 & 0.98 & 1.00 & $0.83^{* *}$ & 1.00 \\
\hline HICP & $0.86^{* *}$ & $0.83^{* *}$ & $0.80^{* *}$ & $0.81^{* *}$ & $0.80^{*}$ & $0.80^{*}$ & $0.81^{*}$ & 0.81 & $0.74^{* * *}$ & $0.74^{*}$ \\
\hline Unemployment & 0.94 & 0.93 & 0.88 & $0.86^{*}$ & $0.84^{* *}$ & $0.82^{* *}$ & $0.80^{* *}$ & $0.79^{* *}$ & $N / A$ & $N / A$ \\
\hline Euribor & 1.02 & 1.02 & 1.00 & 1.01 & 1.01 & 1.00 & 0.99 & 0.97 & $N / A$ & $N / A$ \\
\hline
\end{tabular}

Note: Panel A displays the raw BVAR in levels CRPS. Panels B to E show the ratios of the CRPS of the entropic tilted BVAR in levels to the raw BVAR in levels. Numbers in bold imply that the tilted BVAR in levels improves over the raw BVAR in levels. Asterisks *, ** and ${ }^{* * *}$ imply statistical significance of a two-sided Diebold and Mariano (1995) test of equal predictive ability at the $10 \%, 5 \%, 1 \%$ level. The variance is estimated using Bartlett kernel weights with bandwidth equal to the forecast horizon $h$. The evaluation sample size is $P=72$ quarters. The columns $h=4^{*}$ and $h=8^{*}$ show the results for the one- and two-year-ahead, year-on-year predictions, respectively. 
Table C.18: Soft Conditioning versus BVAR in levels: RMSFE

\begin{tabular}{|c|c|c|c|c|c|c|c|c|c|c|}
\hline \multirow[t]{2}{*}{ Forecast horizon $l$} & $h=1$ & $h=2$ & $h=3$ & $h=4$ & $h=5$ & $h=6$ & $h=7$ & $h=8$ & $h=4 *$ & $h=8 *$ \\
\hline & \multicolumn{10}{|c|}{ Panel A. BVAR } \\
\hline GDP & 0.54 & 0.58 & 0.58 & 0.57 & 0.56 & 0.57 & 0.56 & 0.56 & 1.67 & 1.88 \\
\hline HICP & 0.29 & 0.32 & 0.35 & 0.36 & 0.36 & 0.36 & 0.36 & 0.36 & 1.15 & 1.30 \\
\hline Unemployment & 0.27 & 0.42 & 0.60 & 0.79 & 0.98 & 1.17 & 1.35 & 1.53 & $N / A$ & $N / A$ \\
\hline \multirow[t]{2}{*}{ Euribor } & 0.40 & 0.76 & 1.10 & 1.43 & 1.73 & 2.00 & 2.24 & 2.46 & $N / A$ & $N / A$ \\
\hline & \multicolumn{10}{|c|}{ Panel B. Soft Conditioning GDP } \\
\hline GDP & $0.88^{*}$ & $0.91^{* *}$ & $0.95^{* *}$ & 0.99 & 1.01 & 1.02 & 1.03 & 1.03 & $0.89^{*}$ & 1.01 \\
\hline HICP & 0.99 & 1.00 & 1.00 & 1.00 & 1.00 & 1.01 & 1.02 & 1.01 & 1.00 & 1.01 \\
\hline Unemployment & 0.97 & 0.94 & $0.94^{*}$ & $0.94^{* *}$ & $0.94^{* *}$ & $0.94^{* * *}$ & $0.95^{* *}$ & $0.95^{* *}$ & $N / A$ & $N / A$ \\
\hline \multirow[t]{2}{*}{ Euribor } & 1.01 & 1.00 & 0.99 & 0.99 & 0.98 & 0.98 & 0.98 & 0.98 & $N / A$ & $N / A$ \\
\hline & \multicolumn{10}{|c|}{ Panel C. Soft Conditioning HICP } \\
\hline GDP & 0.99 & 0.98 & 0.99 & 0.99 & 1.00 & 1.01 & 1.01 & 1.02 & 0.98 & 1.00 \\
\hline HICP & $0.90^{* *}$ & $0.89^{* *}$ & $0.86^{* *}$ & $0.86^{* *}$ & $0.85^{*}$ & $0.84^{*}$ & $0.84^{*}$ & $0.83^{* *}$ & $0.83^{* *}$ & $0.79^{* *}$ \\
\hline Unemployment & 0.98 & 0.98 & 0.98 & 0.99 & 0.99 & 0.99 & 1.00 & 1.00 & $N / A$ & $N / A$ \\
\hline \multirow[t]{2}{*}{ Euribor } & 0.99 & 1.00 & 1.00 & 1.00 & 0.99 & 0.99 & 0.98 & 0.97 & $N / A$ & $N / A$ \\
\hline & \multicolumn{10}{|c|}{ Panel D. Soft Conditioning Unemployment } \\
\hline GDP & 0.98 & 0.98 & 0.99 & 0.99 & 0.98 & $0.98^{* *}$ & 0.98 & 0.98 & 0.98 & $0.97^{* *}$ \\
\hline HICP & 0.99 & 0.99 & 0.99 & 0.99 & 0.99 & 0.99 & 0.99 & 1.00 & 0.98 & 0.99 \\
\hline Unemployment & 0.99 & $0.97^{*}$ & $0.96^{*}$ & $0.96^{*}$ & $0.95^{* *}$ & $0.95^{* * *}$ & $0.94^{* * *}$ & $0.93^{* * *}$ & $N / A$ & $N / A$ \\
\hline \multirow[t]{2}{*}{ Euribor } & 1.01 & 1.01 & 1.01 & 1.01 & 1.01 & 1.01 & 1.00 & 0.99 & $N / A$ & $N / A$ \\
\hline & \multicolumn{10}{|c|}{ Panel E. Soft Conditioning GDP, HICP and Unemployment } \\
\hline GDP & $0.88^{*}$ & $0.91^{*}$ & $0.95^{* *}$ & 0.98 & $1.00^{\circ}$ & 1.01 & 1.02 & 1.02 & $0.88^{*}$ & 1.00 \\
\hline HICP & $0.89^{* *}$ & $0.89^{* *}$ & $0.87^{* *}$ & $0.87^{* *}$ & $0.86^{*}$ & $0.86^{*}$ & $0.86^{*}$ & $0.85^{*}$ & $0.83^{* *}$ & $0.81^{*}$ \\
\hline Unemployment & 0.96 & $0.93^{* *}$ & $0.92^{*}$ & $0.92^{*}$ & $0.93^{* *}$ & $0.93^{* * *}$ & $0.93^{* * *}$ & $0.92^{* * *}$ & $N / A$ & $N / A$ \\
\hline Euribor & 1.00 & 1.00 & 0.99 & 0.99 & 0.98 & 0.97 & 0.96 & 0.94 & $N / A$ & $N / A$ \\
\hline
\end{tabular}

Note: Panel A displays the raw BVAR in levels RMSFE. Panels B to E show the ratios of the RMSFE of the soft conditioned BVAR in levels to the raw BVAR in levels. Numbers in bold imply that the soft conditioned BVAR in levels improves over the raw BVAR in levels. Asterisks *, ${ }^{* *}$ and ${ }^{* * *}$ imply statistical significance of a two-sided Diebold and Mariano (1995) test of equal predictive ability at the $10 \%, 5 \%, 1 \%$ level. The variance is estimated using Bartlett kernel weights with bandwidth equal to the forecast horizon $h$. The evaluation sample size is $P=72$ quarters. The columns $h=4^{*}$ and $h=8^{*}$ show the results for the one- and two-year-ahead, year-on-year predictions, respectively. 
Table C.19: Soft Conditioning versus BVAR in levels: CRPS

\begin{tabular}{|c|c|c|c|c|c|c|c|c|c|c|}
\hline \multirow[t]{2}{*}{ Forecast horizon $l$} & $h=1$ & $h=2$ & $h=3$ & $h=4$ & $h=5$ & $h=6$ & $h=7$ & $h=8$ & $h=4 *$ & $h=8 *$ \\
\hline & \multicolumn{10}{|c|}{ Panel A. BVAR } \\
\hline GDP & 0.26 & 0.27 & 0.28 & 0.27 & 0.28 & 0.27 & 0.27 & 0.27 & 0.81 & 1.05 \\
\hline HICP & 0.16 & 0.18 & 0.20 & 0.21 & 0.20 & 0.20 & 0.21 & 0.21 & 0.67 & 0.76 \\
\hline Unemployment & 0.16 & 0.24 & 0.36 & 0.49 & 0.63 & 0.76 & 0.90 & 1.03 & $N / A$ & $N / A$ \\
\hline \multirow[t]{2}{*}{ Euribor } & 0.19 & 0.39 & 0.58 & 0.78 & 0.96 & 1.11 & 1.26 & 1.38 & $N / A$ & $N / A$ \\
\hline & \multicolumn{10}{|c|}{ Panel B. Soft Conditioning GDP } \\
\hline GDP & $0.89^{* * *}$ & $0.90^{* * *}$ & $0.93^{* * *}$ & $0.95^{*}$ & 0.97 & 0.98 & 0.99 & 0.99 & $0.92^{* *}$ & 1.04 \\
\hline HICP & 1.00 & 1.00 & 1.01 & 1.00 & 1.01 & 1.02 & 1.03 & 1.02 & 1.01 & 1.04 \\
\hline Unemployment & 0.99 & 0.97 & $0.96^{*}$ & $0.96^{*}$ & $0.96^{*}$ & $0.97^{*}$ & 0.97 & 0.98 & $N / A$ & $N / A$ \\
\hline \multirow[t]{2}{*}{ Euribor } & 1.01 & 1.02 & 1.02 & 1.02 & 1.02 & 1.01 & 1.01 & 1.00 & $N / A$ & $N / A$ \\
\hline & \multicolumn{10}{|c|}{ Panel C. Soft Conditioning HICP } \\
\hline GDP & 0.99 & 0.99 & 0.98 & 0.99 & 0.99 & 1.00 & 1.01 & 1.01 & 0.99 & 1.01 \\
\hline HICP & $0.91^{* *}$ & $0.90^{*}$ & $0.88^{* *}$ & $0.88^{* *}$ & $0.87^{*}$ & $0.86^{*}$ & $0.85^{*}$ & $0.84^{*}$ & $0.88^{*}$ & 0.86 \\
\hline Unemployment & 0.99 & 1.00 & 1.01 & 1.01 & 1.02 & $1.02^{*}$ & $1.02^{* *}$ & $1.02^{* *}$ & $N / A$ & $N / A$ \\
\hline \multirow[t]{2}{*}{ Euribor } & 1.00 & 1.02 & 1.02 & 1.03 & 1.02 & 1.02 & 1.01 & 1.00 & $N / A$ & $N / A$ \\
\hline & \multicolumn{10}{|c|}{ Panel D. Soft Conditioning Unemployment } \\
\hline GDP & 0.99 & 0.98 & 0.97 & $0.97^{* *}$ & $0.96^{* * *}$ & $0.96^{* *}$ & $0.96^{* *}$ & $0.96^{* *}$ & 0.99 & $0.96^{*}$ \\
\hline $\mathrm{HICP}$ & 0.99 & 0.98 & 0.99 & 0.99 & 0.99 & 0.99 & 1.00 & 1.00 & 0.99 & 1.00 \\
\hline Unemployment & 1.00 & 0.99 & 0.98 & 0.98 & 0.98 & 0.98 & 0.97 & $0.97^{*}$ & $N / A$ & $N / A$ \\
\hline \multirow[t]{2}{*}{ Euribor } & 1.01 & 1.01 & 1.01 & 1.01 & 1.01 & 1.01 & 1.01 & 1.00 & $N / A$ & $N / A$ \\
\hline & \multicolumn{10}{|c|}{ Panel E. Soft Conditioning GDP, HICP and Unemployment } \\
\hline GDP & $0.89^{* * *}$ & $0.89^{* * *}$ & $0.92^{* * *}$ & $0.95^{* * *}$ & $0.97^{\circ}$ & 0.98 & 0.98 & 0.99 & $0.92^{*}$ & 1.04 \\
\hline HICP & $0.91^{* *}$ & $0.91^{*}$ & $0.89^{*}$ & $0.89^{*}$ & 0.89 & 0.88 & 0.88 & 0.87 & 0.89 & 0.89 \\
\hline Unemployment & 0.99 & 0.98 & 0.97 & 0.97 & 0.98 & 0.98 & 0.98 & 0.99 & $N / A$ & $N / A$ \\
\hline Euribor & 1.01 & 1.04 & 1.04 & 1.04 & 1.04 & 1.03 & 1.01 & 1.00 & $N / A$ & $N / A$ \\
\hline
\end{tabular}

Note: Panel A displays the raw BVAR in levels CRPS. Panels B to E show the ratios of the CRPS of the soft conditioned BVAR in levels to the raw BVAR in levels. Numbers in bold imply that the soft conditioned BVAR in levels improves over the raw BVAR in levels. Asterisks *, ** and ${ }^{* * *}$ imply statistical significance of a two-sided Diebold and Mariano (1995) test of equal predictive ability at the $10 \%, 5 \%, 1 \%$ level. The variance is estimated using Bartlett kernel weights with bandwidth equal to the forecast horizon $h$. The evaluation sample size is $P=72$ quarters. The columns $h=4^{*}$ and $h=8^{*}$ show the results for the one- and two-year-ahead, year-on-year predictions, respectively. 